\author{
Universidade Federal da Paraíba \\ Universidade Federal de Campina Grande \\ Programa Associado de Pós-Graduação em Matemática \\ Doutorado em Matemática
}

\title{
Qualitative properties of positive singular solutions to nonlinear elliptic systems with critical exponent
}

\author{
por \\ Rayssa Helena Aires de Lima Caju
}

João Pessoa - PB

Fevereiro/2018 


\section{Qualitative properties of positive singular solutions to nonlinear elliptic systems with critical exponent}

por

Rayssa Helena Aires de Lima Caju用

sob orientação do

Prof. Dr. João Marcos Bezerra do Ó

e coorientação do

Prof. Dr. Fernando Codá dos Santos Cavalcanti Marques

Tese apresentada ao Corpo Docente do Programa Associado de Pós-Graduação em Matemática UFPB/UFCG, como requisito parcial para obtenção do título de Doutor em Matemática.

\section{João Pessoa - PB}

Fevereiro/2018

${ }^{\dagger}$ Este trabalho contou com apoio financeiro da CAPES 
Catalogação na publicação

Seção de Catalogação e Classificação

C139q Caju, Rayssa Helena Aires de Lima.

Qualitative properties of positive singular solutions to nonlinear elliptic systems with critical exponent / Rayssa Helena Aires de Lima Caju. - João Pessoa, 2018.

90 f. : il.

Orientação: João Marcos Bezerra do ó.

Coorientação: Fernando Codá dos Santos Cavalcanti Marques.

Tese (Doutorado) - UFPB/CCEN.

1. Matemática. 2. Equações de Schrödinger. 3.

Comportamento assintótico. I. ó, João Marcos Bezerra do. II. Marques, Fernando Codá dos Santos Cavalcanti. III. Título.

$\mathrm{UFPB} / \mathrm{CCSA}$ 


\section{Universidade Federal da Paraíba \\ Universidade Federal de Campina Grande \\ Programa Associado de Pós-Graduação em Matemática \\ Doutorado em Matemática}

Área de Concentração: Análise

Aprovada em: 23 de fevereiro de 2018

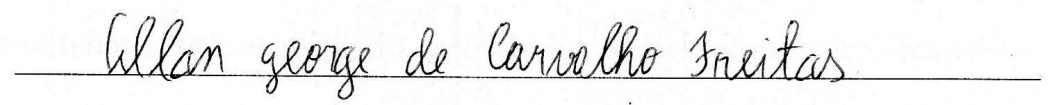

Prof. Dr. Allan George de Carvalho Freitas

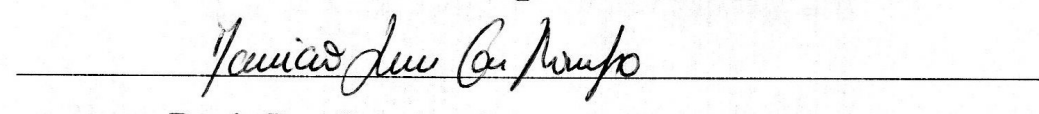

Prof. Dr. Damião Júnio Gonçalves Araújo

Prof. Dra. Marta Calanchi

Prof. Dr. Pedro Eduardo Ubilla Lopez

Prof. Dr. Fernando Codá dos Santos Cavalcanti Marques

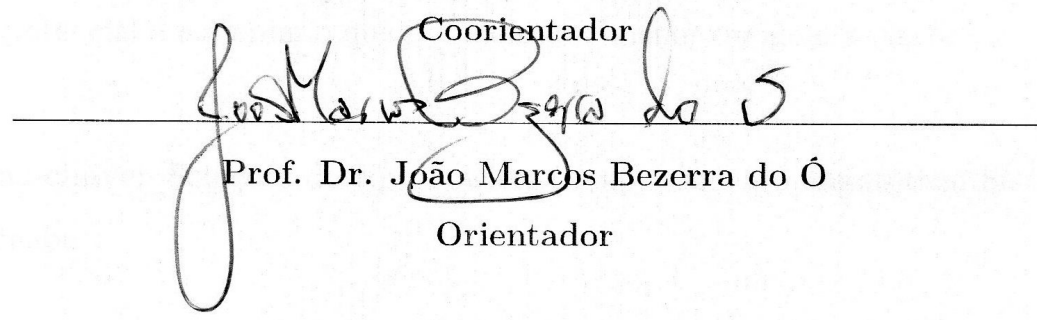

Tese apresentada ao Corpo Docente do Programa Associado de Pós-Graduação em Matemática - UFPB/UFCG, como requisito parcial para obtenção do título de Doutor em Matemática.

Fevereiro/2018 


\section{Resumo}

Neste trabalho estudaremos o comportamento assintótico de soluções positivas do seguinte sistema elípticos acoplado de equações de Schrödinger não lineares

$$
\Delta_{g} u_{i}-\sum_{j=1}^{2} A_{i j}(x) u_{j}+\frac{n(n-2)}{4}|\mathcal{U}|^{\frac{4}{n-2}} u_{i}=0
$$

definido em $B_{1}(0) \backslash\{0\}$ para $n \geq 3$, onde $g$ é uma métrica Riemanniana na bola unitária e o potential $A$ é um mapa de classe $C^{1}$ tal que $A_{i j}(x)$ é uma matriz simétrica para cada $x$ pertencente a $B_{1}(0)$. Do ponto de vista da geometria conforme, o sistema acima é uma extensão natural de equações do tipo Yamabe.

Abordaremos o problema assumindo primeiramente que $g$ é a métrica euclidiana e que o potencial $A$ é identicamente nulo. Nesse caso iremos provar que as soluções do nosso problema são assintóticas ao que chamaremos de soluções do tipo Fowler. No caso geral, iremos demonstrar que o mesmo resultado inserindo algumas restrições sobre o potencial e assumindo que a dimensão é menor ou igual a cinco.

Palavras-chave: Soluções do tipo Fowler; Comportamento assintótico; Sistemas do tipo Yamabe. 


\section{Abstract}

In this work we study the asymptotic behavior to positive solutions of the following coupled elliptic system of nonlinear Schrödinger equations

$$
\Delta_{g} u_{i}-\sum_{j=1}^{2} A_{i j}(x) u_{j}+\frac{n(n-2)}{4}|\mathcal{U}|^{\frac{4}{n-2}} u_{i}=0
$$

which are defined in the punctured unit ball $B_{1}(0) \backslash\{0\}$ for $n \geq 3$. Here $g$ is a Riemannian metric on the unit ball and the potential $A$ is assumed a $C^{1}$ map such that $A_{i j}(x)$ is a symmetrical matrix for each $x$ in $B_{1}(0)$. From the viewpoint of conformal geometry, this systems are pure extensions of Yamabe-type equations.

We will approach the problem assuming first that $g$ is the euclidian metric and the potential $A$ vanishes. In this case we are able to prove that the solutions of our problem are asymptotics to what we call Fowler-type solutions. In the general case we will prove the same result by putting some restrictions on the potential and assuming that the dimension is less or equal to five.

Keywords: Fowler-type solutions; Asymptotic behavior; Yamabe-type system. 


\section{Agradecimentos}

\section{A Deus.}

Ao Professor João Marcos Bezerra do Ó por ter orientado este trabalho, pelo conhecimento transmitido, por me motivar a enfrentar novos desafios e, principalmente, pela confiança depositada.

A Professora Flávia Jerônimo pela ajuda nos momentos de dificuldade, apoio, incentivo, confiança, e por ter acreditado em mim sempre.

Ao Professor Fernando Codá Marques por ter me recebido em Princeton, pela paciência, pelo encorajamento e por todo o conhecimento transmitido.

Ao Professor Almir Silva Santos pela colaboração e por inúmeras contribuições.

A minha família, em especial a minha avó, pela cobrança, carinho, apoio e ajuda em todas as decisões.

Ao meu companheiro Pedro Gaspar pelo encorajamento, apoio, confiança e paciência durante os momentos mais obscuros dessa jornada.

Aos meus amigos ao longo dessa caminhada na UFPB em especial à Ageu, Diego, Ellen, Eudes, José Carlos, Leon, Rainelly, Ricardo e Victor pela amizade e companheirismo. A turma brasileira em Princeton.

Aos professores da UFPB, em especial a Antônio Sales e Sergio de Albuquerque, que me receberam tão jovem na universidade e aos professores Alexandre Simas, Everaldo Souto, Jacqueline Rojas, Manassés Xavier, Sergio de Albuquerque e Uberlândio Severo, que acompanharam toda a minha trajetória a partir da graduação.

A banca examinadora: Prof. por aceitarem participar da avaliação deste trabalho.

A CAPES - Coordenação de Aperfeiçoamento de Pessoal de Nível superior, pelo apoio financeiro. 
"Sou metade agonia, metade esperança."

Jane Austen

viii 


\section{Dedicatória}

A minha avó e ao meu noivo. 


\section{Contents}

Introduction . . . . . . . . . . . . . . . . . . . . . . 1

Notation and terminology $\ldots \ldots \ldots \ldots \ldots \ldots$

\begin{tabular}{lll}
\hline 1 & Fowler solutions and Jacobi fields & 7
\end{tabular}

1.1 The linearized equation $\ldots \ldots \ldots \ldots \ldots$

$\begin{array}{|lll|}2 & \text { Classification result for the Limit system } & 11\end{array}$

2.1 Radial Symmetry . . . . . . . . . . . . . . . . . . . . . . . . . . . 12

2.2 ODE Analysis . . . . . . . . . . . . . . . . . . . . . . . . . . . . . 14

2.3 Removable singularity result . . . . . . . . . . . . . . . . . . . . . . . . 16

2.4 Classification Result $\ldots \ldots \ldots \ldots$

2.5 Jacobi Fields for the Limit System . . . . . . . . . . . . . . . . . . . . 21

\begin{tabular}{|lll}
3 & Coupled elliptic system in the punctured ball & 25
\end{tabular}

3.1 Upper bound $\ldots \ldots \ldots \ldots \ldots$. . . . . . . . . . . . . . . . . . . . 25

3.2 Pohozaev invariant and lower bound f . . . . . . . . . . . . . . . 33

3.3 Simple convergence to a radial solution . . . . . . . . . . . . . . . . 38

4 Yamabe-type system in the punctured ball 45

4.1 Upper bound near a singularity . . . . . . . . . . . . . . . . 45

$4.2 \quad$ Pohozaev invariants and removable singularities . . . . . . . . . . . . 56

4.3 Convergence to a Radial Solution . . . . . . . . . . . . . . . . . . . . 70

\begin{tabular}{ll}
\hline References & 78
\end{tabular} 


\section{List of Figures}

1.1 Level sets $\ldots \ldots \ldots \ldots \ldots$ 


\section{Introduction}

In this thesis our main purpose is to study the asymptotic behavior for positive solutions to the following coupled elliptic system of nonlinear Schrödinger equations

$$
\Delta_{g} u_{i}-\sum_{j=1}^{2} A_{i j}(x) u_{j}+\frac{n(n-2)}{4}|\mathcal{U}|^{\frac{4}{n-2}} u_{i}=0
$$

which are defined in the punctured ball $\Omega=B_{1}(0) \backslash\{0\}$, where $g$ is a smooth Riemannian metric on the unit ball $B_{1}(0) \subset \mathbb{R}^{n}, n \geq 3$ and $|\mathcal{U}|^{2}=u_{1}^{2}+u_{2}^{2}$. Here $A$ is a $C^{1}$ map from $B_{1}(0)$ to the vector space of symmetrical $2 \times 2$ real matrices $M_{2}^{s}(\mathbb{R})$, where we will write $A=\left(A_{i j}\right)_{i, j}$ and each $A_{i j}$ is a $C^{1}$ real value function.

The critical system (1) is weakly coupled by the matrix $A$, and strongly coupled by the Gross-Pitaevskii type nonlinearity in the right-hand side of (1). Systems involving this type of nonlinearities are strongly related to two branches of mathematical physics. They appear in the Hartree-Fock theory for double condensates, a binary mixture of Bose-Einstein condensates in two different hyperfine states and they also arise in the study of incoherent solitons in nonlinear optics. We refer the reader to [1], [2] and [8].

In the last few years there has been considerable interest in this type of systems. O. Druet, E. Hebey and J. Vetóis in [7] studied stability properties for systems of the form (1) in a compact Riemannian manifold when the potential $A$ is less, in the sense of bilinear forms, than the geometric threshold potential of the conformal laplacian. Consequently, from the viewpoint of conformal geometry, our systems are pure extensions of Yamabe-type equations in the strongly coupled regime and the consideration of such elliptic systems provides a natural background for the interplay between geometry and asymptotic analysis.

Yamabe-type problems are also widely studied in the singular case, in which one is 
interested in finding complete conformal metrics with constant scalar curvature in noncompact manifolds with a simple structure at infinity. Having in mind the connections between geometry and asymptotic analysis it is natural to study properties such as existence and the asymptotic behavior of solutions to the system (1) in the singular case.

Recall that, from the analytic viewpoint, the simplest instance of the singular Yamabe problem consists in finding positive solutions to

$$
\Delta_{g} u-\frac{n-2}{4(n-1)} R_{g} u+\frac{n(n-2)}{4} u^{\frac{n+2}{n-2}}=0,
$$

in the punctured unit ball endowed with a metric $g$ which blows-up at the origin.

The case of the flat metric was first studied by L. Caffarelli, B. Gidas and J. Spruck in [3] in the punctured unit ball and they proved that, when 0 is a nonremovable singularity, the local models are given by the radial Fowler solutions. Remember that $u_{0}$ is a Fowler solution if it satisfies

$$
\Delta u_{0}+\frac{n(n-2)}{4} u_{0}^{\frac{n+2}{n-2}}=0 \quad \text { in } \quad \mathbb{R}^{n} \backslash\{0\},
$$

which blows-up at origin. Their proof relies on a complicated version of the Alexandrov reflection method, and it was later simplified by N. Korevaar et al in [11].

We begin by considering the system (1) in its simplest form, which will be a natural generalization of the singular Yamabe problem in the flat case. Assuming that the matrix $A$ is identically zero and that $\mathrm{g}$ is the euclidian metric, the system (1) becomes

$$
\Delta u_{i}+\frac{n(n-2)}{4}|\mathcal{U}|^{\frac{4}{n-2}} u_{i}=0
$$

in the punctured ball $B_{1}^{n}(0) \backslash\{0\}$.

Firstly we describe what we mean by a positive singular solution to the general system (1), in particular to (4). We say that $\mathcal{U}$ is a positive solution if each coordinate $u_{i}$ is positive and that it is singular if the following holds

$$
\lim _{|x| \rightarrow 0}|\mathcal{U}|(x)=+\infty
$$

Our main goal is to prove that singular solutions of (4) are asymptotic to what we call Fowler-type solutions, that is, solutions of the limit system

$$
\Delta u_{i}+\frac{n(n-2)}{4}|\mathcal{U}|^{\frac{4}{n-2}} u_{i}=0 \quad \text { in } \quad \mathbb{R}^{n} \backslash\{0\}
$$


Our main result for system (4) is the following

Theorem 0.1 Suppose that $\mathcal{U}$ is a solution of the system (4) in the punctured ball $B_{1}^{n}(0) \backslash\{0\}$. Then there exists a Fowler-type solution $\mathcal{U}_{0}=u_{0} \Lambda$ of (6), where $u_{0}$ is a Fowler solution such that

$$
\mathcal{U}(x)=\left(1+O\left(|x|^{\alpha}\right)\right) \mathcal{U}_{0}(x)
$$

as $x \rightarrow 0$, for some $\alpha>0$.

In light of the analogy between the singular problem (2) and the system (1), we are led to analyze the properties of our local model, the Fowler-type solutions.

It was proved by the authors in [7] the following classification result for entire solutions of the system

$$
\Delta u_{i}+\frac{n(n-2)}{4}|\mathcal{U}|^{\frac{4}{n-2}} u_{i}=0 \quad \text { in } \quad \mathbb{R}^{n}
$$

Theorem A [7] Let $\mathcal{U}$ be a nonnegative $C^{2}$-solution of (8). Then there exist $x_{0} \in \mathbb{R}^{n}$, $\mu>0$, and $\Lambda \in \mathbb{S}_{+}^{1}$, such that

$$
\mathcal{U}(x)=\left(\frac{2 \mu}{1+\mu^{2}\left|x-x_{0}\right|^{2}}\right)^{\frac{n-2}{2}} \Lambda
$$

for all $x \in \mathbb{R}^{n}$, where $\mathbb{S}_{+}^{1}$ consists of the elements in the unit sphere in $\mathbb{R}^{p}$, with nonnegative coordinates.

This result inspired us to ask whether any singular nonnegative solution is of the form $u_{0} \Lambda$, where now $u_{0}$ is a Fowler solution and $\Lambda$ is a vector in the unit sphere with nonnegative coordinates. Indeed, we are able to prove the following result

Theorem 0.2 Suppose that $\mathcal{U}_{0}$ is a nonnegative singular solution for (6). Then there exist $\Lambda \in \mathbb{S}_{+}^{1}$ and $u_{0}$ a Fowler solution such that

$$
\mathcal{U}_{0}=u_{0} \Lambda
$$

where $\mathbb{S}_{+}^{1}$ consists of the elements in the unit sphere in $\mathbb{R}^{2}$, with nonnegative coordinates.

Classification results for singular solutions for coupled elliptic systems were conjectured by Z. Chen and C-S. Lin in [5] for a similar system. This classification will allow us to study all the properties that we need for solutions of the limit system based in the properties of Fowler solution. 
Using this classification result, the strategy to prove that the solutions of (4) are asymptotic to Fowler-type solution is to get an upper and lower bound. The upper bound will follow from a more precise universal bound for weak supersolutions which are smooth in a open set which is inspired by the works of [18, [16] and [12]. The lower bound depends in a more delicate way of the solution. This finishes the study of the solutions of (4).

While Korevaar et al. [11] were able to prove the asymptotic behavior for the singular Yamabe problem in the flat case for any dimensions, for the general problem in the unit ball, F. Marques in [13] has proved the asymptotic behavior when the dimension is less or equal to five. For these dimensions he was able to overcome the lack of symmetry by constructing appropriate auxiliary functions. The problem for higher dimensions is still open.

Once that system (1) can be seen as a natural generalization of the singular Yamabe problem for an arbitrary metric, we need to put the same restrictions for the dimension. Futhermore, sometimes we will consider the following hypotheses on the potential

(H1) $-A$ is cooperative, that is, the components in the nondiagonal $A_{i j}$ of $A, i \neq j$, are nonpositive;

(H2) In dimension $n=5$, there exists a $C^{2}$-function $f$ such that

$$
A(x)=f(x) I d_{2}+O(|x|)
$$

near the origin, where $I d_{2}$ is the identity matrix;

Under these assumptions we are able to prove our main theorem.

Theorem 0.3 Assume $3 \leq n \leq 5$ and let $\mathcal{U}$ be a positive solution for (1) in the punctured unit ball, and suppose that the potential A satisfies (H1) and (H2). If $\mathcal{U}$ has a nonremovable singularity at 0 then there exists a Fowler-type solution $\mathcal{U}_{0}$ of (6) such that

$$
\mathcal{U}(x)=\left(1+O\left(|x|^{\alpha}\right)\right) \mathcal{U}_{0}(x)
$$

as $x \rightarrow 0$, for some $\alpha>0$.

The strategy of the proof of Theorem 0.3 relies on the properties of Fowler solutions. First, using the moving planes technique we prove the upper bound

$$
|\mathcal{U}|(x) \leq c|x|^{\frac{2-n}{2}}
$$


As a consequence of this upper bound we can study Pohozaev integrals for solutions of (11), and use a Pohozaev-type identity to show that the invariant

$$
P(\mathcal{U})=\lim _{r \rightarrow \infty} P(r, \mathcal{U})
$$

is well-defined, where

$$
P(r, \mathcal{U})=\int_{\partial B_{r}}\left(\frac{n-2}{2}\left\langle\mathcal{U}, \frac{\partial \mathcal{U}}{\partial \nu}\right\rangle-\frac{r}{2}|\nabla \mathcal{U}|^{2}+r\left|\frac{\partial \mathcal{U}}{\partial \nu}\right|^{2}+r \frac{(n-2)^{2}}{8}|\mathcal{U}|^{\frac{2 n}{n-2}}\right) d \sigma
$$

Inspired by the works of L. Caffarelli, B. Gidas and J. Spruck in [3], we prove a removable singularity theorem, proving that this invariant is always nonpositive, and it is equal to zero if and only if the singularity is removable. As a consequence of this result we estabilish the lower bound

$$
|\mathcal{U}|(x) \geq c|x|^{\frac{2-n}{2}}
$$

Using these bounds our goal is to use a scaling argument due to Leon Simon to prove Theorem 0.3 . For this purpose, the study of the growth of what we call Jacobi fields for the limit system will be fundamental.

The thesis is organized as follows. In Chapter 1 we are going to do a overview on Fowler solutions and Jacobi fields. In Chapter 2 we will prove the radial symmetry for solutions to the limit system with intent to transform the system (6) in a ODE. Then we prove Theorem 0.2 and study the Jacobi fields associated to (4). In Chapter 3 we prove the upper and lower bounds for solutions of (4) and the asymptotic behavior for solutions of system (4). Finally in Chapter 4.3 we prove Theorem 0.3 assuming the hypothesis (H1) and (H2) on the potential A. 


\section{Notation and terminology}

- $B_{R}(x)$ denotes the open ball of radius $R$ and center $x$;

- $B_{R}=B_{R}(0)$ denotes the open ball of radius $R$ and center 0 ;

- $\chi_{E}$ denotes the characteristic function of a set $E \subset \mathbb{R}^{n}$, that is

$$
\chi_{E}(x)=\left\{\begin{array}{l}
1 \text { if } x \in E \\
0 \text { if } x \in \mathbb{R}^{n} \backslash E
\end{array}\right.
$$

- $o(1)$ denotes a sequence which converges to zero;

- For $1 \leq p<\infty, L^{p}\left(B_{1}(0)\right)^{2}=L^{p}\left(B_{1}(0)\right) \times L^{p}\left(B_{1}(0)\right)$ denotes the Lebesgue space with norm

$$
\|(u, v)\|_{p}^{p}=\|u\|_{p}^{p}+\|v\|_{p}^{p}
$$

- $\mathbb{S}_{+}^{n-1}=\left\{x=\left(x_{1}, \ldots, x_{n}\right) \in \mathbb{S}^{n-1}: x_{i} \geq 0 \quad \forall i\right\}$

- $\Delta_{\mathbb{S}^{n-1}}$ denotes the Laplacian in the unit sphere;

- If $\mathcal{U}=\left(u_{1}, u_{2}\right)$ then $|\mathcal{U}|^{2}=\sum_{i=1}^{2} u_{i}^{2},|\nabla \mathcal{U}|^{2}=\sum_{i=1}^{2} \nabla u_{i}^{2}$ and $\left|\frac{\partial \mathcal{U}}{\partial \nu}\right|^{2}=\sum_{i=1}^{2} \frac{\partial u_{i}{ }^{2}}{\partial \nu}$;

- $g_{c y l}, g_{\text {euc }}$ 


\section{Chapter 1}

\section{Fowler solutions and Jacobi fields}

As mentioned earlier, our main goal in this thesis is to show that the solutions of our system are asymptotic the solutions of the limit system. As these solutions will play a similar role to Fowler's solutions in the case of the singular Yamabe problem, we will briefly review the main properties of Fowler's solutions as well as what we call the Jacobi fields.

We say that a $u$ is a Fowler or Delaunay-type solution if satisfies

$$
\Delta u+\frac{n(n-2)}{4} u^{\frac{n+2}{n-2}}=0 \quad \text { in } \quad \mathbb{R}^{n} \backslash\{0\}
$$

and if it is singular around the origin. Using the Alexandrov reflection argument, the following result of Caffarelli, Gidas and Spruck in [3] characterizes these solutions.

Proposition 1.1 [3] Let $u$ be a positive solution of (1.1). If the origin is a nonremovable singularity, then $u$ is radially symmetric about the origin.

Using that $u$ is rotationally invariant the equation can be reduced to an ODE. Indeed, consider the diffeomorphism

$$
\begin{gathered}
\Phi:\left(\mathbb{R} \times \mathbb{S}^{n-1}, g_{\text {cyl }}=d t^{2}+d \theta^{2}\right) \rightarrow\left(\mathbb{R}^{n} \backslash\{0\}, g_{\text {euc }}\right) \\
\Phi(t, \theta)=e^{-t} \theta .
\end{gathered}
$$

with inverse $\Phi^{-1}(x)=\left(-\log |x|, x|x|^{-1}\right)$ and such that $\Phi^{*} g_{\text {euc }}=e^{-2 t} g_{c y l}$. This diffeomorphism induces the classical change of variables from Fowler, given by

$$
v(t, \theta)=|x|^{\frac{n-2}{2}} u(x)
$$


where $t=-\log |x|$ and $\theta=x /|x|$.

Relative to this change of variables the equation (1.1) is equivalent to

$$
v^{\prime \prime}-\frac{(n-2)^{2}}{4} v+\frac{n(n-2)}{4} v^{\frac{n+2}{n-2}}=0 .
$$

We analyse this equation by converting it into a system of first order equations

$$
\left\{\begin{array}{l}
v^{\prime}=w \\
w^{\prime}=\frac{(n-2)^{2}}{4} v-\frac{n(n-2)}{4} v^{\frac{n+2}{n-2}} .
\end{array}\right.
$$

whose Hamiltonian energy, given by

$$
H(v, w)=w^{2}-\frac{(n-2)^{2}}{4} v^{2}+\frac{(n-2)^{2}}{4} v^{\frac{2 n}{n-2}},
$$

is constant along solutions of (1.3).

By examining the level curves of energy, we see that all positive solutions lie in the bounded set $\{H<0\} \cap\{v>0\}$.

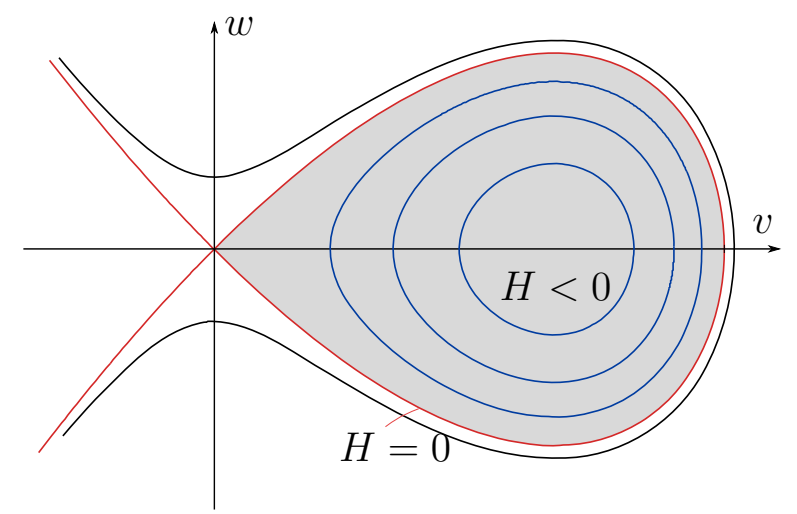

Figure 1.1: Level sets

The basic properties of these solutions are summarized in the next proposition which is proved in [14.

Proposition 1.2 14 For any $H_{0} \in\left(-((n-2) / n)^{n / 2}(n-2) / 2,0\right)$, there exists a unique bounded solution of (1.3) satisfying $H\left(v, v^{\prime}\right)=H_{0}, v^{\prime}(0)=0$ and $v^{\prime \prime}(0)>0$. This solution is periodic, and for all $t \in \mathbb{R}$ we have $v(t) \in(0,1)$. This solution can be indexed by the parameter $\varepsilon=v(0) \in\left(0,((n-2) / n)^{(n-2) / 4}\right)$, which is the smallest of the two values $v$ assumes when $v^{\prime}(0)=0$. When $H_{0}=-((n-2) / n)^{n / 2}((n-2) / 2)$ there is a unique bounded solution of (1.3), given by

$$
v(t)=\left(\frac{n-2}{n}\right)^{\frac{n-2}{4}} .
$$

Finally, if $v$ is a solution with $H_{0}=0$, then either $v(t) \equiv 0$ or $v(t)=\left(\cosh \left(t-t_{0}\right)\right)^{\frac{2-n}{2}}$ for some $t_{0} \in \mathbb{R}$. 


\subsection{The linearized equation}

After introducing the notion of Fowler solutions, we describe some useful properties of the Jacobi fields associated to these solutions. The idea of using Jacobi fields in this context arose initially in the study of the asymptotic behavior of solutions to the conformally invariant semilinear equation

$$
\Delta u+\frac{n(n-2)}{4} u^{\frac{n+2}{n-2}}=0,
$$

which using the cylindrical coordinates change is equivalent to

$$
H(v)=\partial_{t}^{2} v+\Delta_{\mathbb{S}^{n-1}} v-\frac{(n-2)^{2}}{4} v+\frac{n(n-2)}{4} v^{\frac{n+2}{n-2}}=0 .
$$

Note that a solution of the equation (1.4) solves the singular Yamabe problem in the flat case.

Inspired by the work [15], in [11] the authors considered the linearization of the operator in 1.5 around a Fowler solution $v_{\varepsilon}$ given by

$$
L_{\varepsilon}(v)=\left.\frac{\partial}{\partial t}\right|_{t=0} H\left(v_{\varepsilon}+t v\right)=\partial_{t}^{2}+\Delta_{\mathbb{S}^{n-1}}-\frac{(n-2)^{2}}{4}+\frac{n(n+2)}{4} v_{\varepsilon}^{\frac{4}{n-2}} .
$$

Since the operator above has periodic coeficients, it can be studied using the classical Flocquet theory or also by separation of variables, see [17]. Note that this operator can also be defined as the derivative at $s=0$ of $H(U(s))$, where $U(s)$ is any one-parameter family of solutions with $U(0)=v_{\varepsilon}, U^{\prime}(0)=v$.

This linearization was studied using results due to R. Mazzeo, D. Pollack and K. Uhlenbeck in [15] based on the spectral decomposition of the laplacian operator $\Delta_{\mathbb{S}^{n-1}}$. They were able to conclude that $\operatorname{spec}\left(L_{\varepsilon}\right)$ is purely absolutely continuous, with no singular continuous or point spectrum.

If $\left\{\lambda_{k}, \mathcal{X}_{k}(\theta)\right\}$ is the eingendata of $\Delta_{\mathbb{S}^{n-1}}$, using the convention that these eingenvalues are listed with multiplicity, we can write $\lambda_{0}=0, \lambda_{1}=\ldots=\lambda_{n}=n-1$, $\lambda_{n+1}=2 n$ and so on. Hence the linearized operator could be decomposed into many ordinary differential operators given by

$$
L_{\varepsilon, k}=\partial_{t}^{2}+\left(\frac{n(n+2)}{4} v_{\varepsilon}^{\frac{4}{n-2}}-\frac{(n-2)^{2}}{4}-\lambda_{k}\right) .
$$

What we call Jacobi fields are the elements in the kernel of the linearized operator, that is, the functions $\psi$ which satisfy $L_{\varepsilon} \psi=0$. The properties of these fields are important for the spectral analysis of the linearized near origin. 
It is worth noting that considering the linearization of the equation around a Fowler solution is a method used not only to study the asymptotic behavior of the solutions. In fact, the analysis of the linearized operator together with fixed point theorems are the main tools used in the proof of the existence of the singular Yamabe problem, under certain hypotheses, as applied in the works of [20] and [14].

In order to study the properties of the Jacobi fields using the decomposition of the laplacian in terms of the eingenvalues, it is sufficient to consider solutions to the induced problems $L_{\varepsilon, k}\left(\psi_{k}\right)=0$.

It is common, in geometric problems, that some solutions to low values of $k$, not necessarilly all, are obtained as derivatives of one-parameter families of solutions to 1.5. For the first eingenvalue $\lambda_{0}=0$, if we consider the families of solutions to 1.5 . given by

$$
T \rightarrow v_{\varepsilon}(t+T) \quad \text { and } \quad \varepsilon \rightarrow v_{\varepsilon}(t)
$$

and then differentiate these families with respect to the parameters to obtain solutions to 1.7 correspondibg to $\lambda_{0}$ and given by

$$
\psi_{\varepsilon, 0}^{+}(t)=\frac{d}{d T} v_{\varepsilon}(t+T)=v_{\varepsilon}^{\prime}(t+T), \quad \psi_{\varepsilon, 0}^{+}(t)=\frac{d}{d \varepsilon} v_{\varepsilon}(t)
$$

which are linearly independent Jacobi fields with linearly and periodic growth, respectively. They also proved that these two functions form a basis, for each $\varepsilon$, for all temperate solutions of $L_{\varepsilon} \psi=0$ on the cylinder. Any solution of this equation must grow exponentially in one direction or the other.

Using a similar construction for $\lambda_{k}=n-1$, they were able to build linearly independent solutions $\psi_{\varepsilon, k}^{ \pm}$that are exponentially increasing and decreasing. Finally when $k>n+1$, we known that the term of zero order of the above operator is negative, since $\lambda_{k} \geq 2 n$ and $v_{\varepsilon}<1$ which imply that $L_{\varepsilon, k}$ satisfies the maximum principle. 


\section{Chapter 2}

\section{Classification result for the Limit system}

The main goal in this chapter is to study some properties of singular nonnegative solutions $\mathcal{U}=\left(u_{1}, u_{2}\right)$ to the following system

$$
\Delta u_{i}+\frac{n(n-2)}{4}|\mathcal{U}|^{\frac{4}{n-2}} u_{i}=0 \quad \text { in } \quad \mathbb{R}^{n} \backslash\{0\},
$$

where $|\mathcal{U}|^{2}=\sum_{i=1}^{2} u_{i}^{2}$ and $\Delta$ denotes the euclidian laplacian. Similarly to the definition for the system (1), we say that $\mathcal{U}$ is a nonnegative solution if each coordinate $u_{i}$ is nonnegative and we say that it is singular if the following holds

$$
\lim _{|x| \rightarrow 0}|\mathcal{U}|(x)=+\infty
$$

It may happen that only one of the coordinates blows up at the singularity.

The above system will be important since its solutions play a similar role to Fowler solutions in the singular Yamabe problem.

One of our main result in this chapter will be the characterization of the nonnegative singular solutions to the limit system. We will show that all such solutions are Fowler solution times a vector in the unit sphere with nonnegative coordinates (see Theorem 2.10 below). Another important result will be describe when the singularity can be removed in terms of an Pohozaev invariant inspired by the works of Caffarelli, Gidas and Spruck [3] and R. Fowler [9]. We will present two distinct ways of proving this result. 


\section{$2.1 \quad$ Radial Symmetry}

In the first step in this direction we will be using a "measure theoretic" variation of the Alexandrov reflection technique as developed by Gidas, Ni and Nirenberg [10], to prove the radial symmetry of nonnegative singular solutions. To proving this result, we need of the following result from Chen and Lin [4, which ensures that the maximum principle holds for superharmonic functions with isolated sigularities.

Lemma 2.1 [4] Let $E$ be a smooth bounded domain in $\mathbb{R}^{n}$, and $Z$ a compact set in $\mathbb{R}^{n}$ with $\operatorname{Cap}(Z)=0$. Assume that $v(x), h(x)$ are nonnegative continuos functions in $\bar{E} \backslash Z$ and satisfy

$$
\Delta v(x)+h(x) \leq 0 \quad \text { in } \quad E \backslash Z,
$$

in the distributional sense. Then

$$
v(x) \geq \int_{E} G(x, y) h(y) d y+\int_{\partial E} \frac{\partial G(x, y)}{\partial \nu} v(y) d s_{y}
$$

for $x \in E \backslash Z$, where $G(x, y)$ is the Green function of $-\Delta$ in $E$ with the Dirichlet boundary condition. In particular, we have $v(x) \geq \inf _{\partial E \backslash Z} v$.

Theorem 2.2 (Radial Symmetry) Let $n \geq 3$ and $\mathcal{U}=\left(u_{1}, u_{2}\right)$ be a nonnegative $C^{2}$ singular solution of the nonlinear elliptic system (6). Then $\mathcal{U}$ is radially symmetric about the origin.

Proof. Whithout loss of generality, we may assume that $\lim _{|x| \rightarrow 0} u_{1}(x)=+\infty$. Now, we will use the moving planes method.

Fix an arbitrary $z \neq 0$ in $\mathbb{R}^{n}$. Define the Kelvin transform

$$
U_{i}(x)=\frac{1}{|x|^{n-2}} u_{i}\left(z+\frac{x}{|x|^{2}}\right)
$$

for $i \in\{1,2\}$. By Caffarelli et al. [3], the Kelvin transform $U_{i}$ has the asymptotic expansion at infinity [3, Lemma 2.3]. Since 0 is a nonremovable singularity for $u_{1}$ we can see that $U_{1}$ is singular at 0 and $z_{0}=-z /|z|^{2}$, and $U_{2}$ is singular at 0 . Moreover, the Kelvin transform satisfies

$$
-\Delta U_{i}=\frac{n(n-2)}{4}|\mathbf{U}|^{\frac{4}{n-2}} U_{i} \quad \text { in } \quad \mathbb{R}^{n} \backslash\left\{0, z_{0}\right\},
$$

where $|\mathbf{U}|^{2}=\sum_{i=1}^{2} U_{i}^{2}$. It is easy to see that

$$
U_{i}(x)=O\left(|x|^{2-n}\right) \quad \text { as } \quad|x| \rightarrow \infty
$$


and that each $U_{i}$ has the following harmonic asymptotic expansion at infinity

$$
\begin{gathered}
U_{i}(x)=a_{i 0}|x|^{2-n}+a_{i j} x_{j}|x|^{-n}+O\left(|x|^{-n}\right), \\
\partial_{x_{j}} U_{i}=(2-n) a_{i 0} x_{j}|x|^{-n}+O\left(|x|^{-n}\right)
\end{gathered}
$$

and

$$
\partial_{x_{k}} \partial_{x_{j}} U_{i}=O\left(|x|^{-n}\right),
$$

where $a_{i 0}=u_{i}(z)$ and $a_{i j}=\partial_{y_{j}} u_{i}(z)$.

We will show that $U_{i}$ are axisymmetric with respect to the axis going through 0 and $z$. Choose a reflection direction orthogonal to this axis and assume without loss of generality that it is equal to the positive $x_{n}$ direction $(0, \ldots, 0,1)$. For $\lambda>0$ let $\Sigma_{\lambda}:=\left\{x \in \mathbb{R}^{n}: x_{n}>\lambda\right\}$ and consider the reflection

$$
x=\left(x_{1}, \ldots, x_{n-1}, x_{n}\right) \in \Sigma_{\lambda} \mapsto x_{\lambda}=\left(x_{1}, \ldots, x_{n-1}, 2 \lambda-x_{n}\right) .
$$

Using again the asymptotic expansion at infiniy for the Kelvin transform from [3], there exist positive constants $\bar{\lambda}>10$ and $R>\left|z_{0}\right|+10$ such that for any $\lambda \geq \bar{\lambda}$ we have, for each coordinate

$$
U_{i}(x)<U_{i}\left(x_{\lambda}\right) \text {, for } x \in \Sigma_{\lambda} \text { and }\left|x_{\lambda}\right|>R \text {. }
$$

By Lemma 2.1, there exists a constant $C>0$ such that

$$
U_{i}(x) \geq C, \quad \text { for } \quad x \in \bar{B}_{R} \backslash\left\{0, z_{0}\right\}
$$

Since $U_{i}(x) \rightarrow 0$ as $|x| \rightarrow+\infty$, there exists $\lambda_{0}>\bar{\lambda}$ such that when $|x| \geq 2 \lambda_{0}-R$ it holds $U_{i}(x)<C$. On the other hand if $x \in \Sigma_{\lambda}$ and $x_{\lambda} \in \bar{B}_{R}$ then $x \notin B_{2 \lambda-R}$. Thus for every $\lambda \geq \lambda_{0}$ we have

$$
U_{i}(x) \leq U_{i}\left(x_{\lambda}\right) \text {, for all } x \in \Sigma_{\lambda} \text { s.t. } x_{\lambda} \notin\left\{0, z_{0}\right\} \text {. }
$$

Let

$$
\lambda^{*}:=\inf \{\tilde{\lambda}>0 \mid 2.3 \text { holds for all } \lambda \geq \tilde{\lambda}\} .
$$

It suffices to show that $\lambda^{*}=0$. Indeed, this proves that $U_{i}$ is axisymmetric with respect to the axis going through 0 and $z$ and since $z$ is arbitrary, each $u_{i}$ is radially symmetric about the origin. 
Suppose by contradiction that $\lambda^{*}>0$. Then (2.3) holds for $\lambda=\lambda^{*}$. Since $U_{1}$ blows-up at $z_{0}$ we see that $U_{1}$ cannot be invariant by the reflection $x_{\lambda^{*}}$. Then by applying the maximum principle to $U_{1}\left(x_{\lambda^{*}}\right)-U_{1}(x)$ and $U_{2}\left(x_{\lambda^{*}}\right)-U_{2}(x)$ in $(2.2)$, we conclude that

$$
U_{i}(x)<U_{i}\left(x_{\lambda}\right) \text {, for } x \in \Sigma_{\lambda} \text { and } x_{\lambda} \notin\left\{0, z_{0}\right\} .
$$

Then applying the Hopf boundary lemma for $x \in \partial \Sigma_{\lambda^{*}}$ we obtain

$$
\partial_{x_{n}}\left(U_{i}\left(x_{\lambda^{*}}\right)-U_{i}(x)\right)=-2 \partial_{x_{n}} U_{i}(x)>0
$$

Now choose sequences $\lambda_{j} \nearrow \lambda^{*}$ and $x^{j} \in \Sigma_{\lambda_{j}}$ such that $U_{1}\left(x_{\lambda_{j}}^{j}\right)<U_{1}\left(x^{j}\right)$. By [3. Lemma 2.4] (the plane $x_{n}=0$ there corresponds to $x_{n}=\lambda^{*}$ here), we conclude that the sequence $\left|x^{j}\right|$ is bounded. Hence passing to a subsequence we may assume that $x_{j} \rightarrow \bar{x} \in \Sigma_{\lambda^{*}}$ with $U_{1}\left(\bar{x}_{\lambda^{*}}\right) \leq U_{1}(\bar{x})$. By 2.4 we know that $\bar{x} \in \partial \Sigma_{\lambda^{*}}$ and then $\partial_{x_{n}} U_{1}(\bar{x}) \geq 0$, a contradiction with (2.5). Therefore, $\lambda^{*}=0$.

Once we proved the radial symmetry of the solutions we may use the classical change of variables from Fowler to approach our problems using ODE analysis. This is the subject of the next section.

\subsection{ODE Analysis}

If $\mathcal{U}=\left(u_{1}, u_{2}\right)$ is a solution of the system, from (2.2) we may assume that each $u_{i}(x)=u_{i}(|x|)$. So using the classical change of variables from Fowler 1.2$)$, it follows that $V=\left(v_{1}, v_{2}\right)$ satisfies the ordinary differential equation

$$
\left\{\begin{array}{c}
v_{i}^{\prime \prime}-\delta^{2} v_{i}+\frac{n(n-2)}{4}|V|^{\frac{4}{n-2}} v_{i}=0, \quad t \in \mathbb{R} \\
v_{i} \geq 0, \quad v_{i} \in C^{2}(\mathbb{R}) .
\end{array}\right.
$$

where $\delta=(n-2) / 2$. Define

$$
\Psi(t):=\frac{1}{2}\left(\left|V^{\prime}\right|^{2}-\delta^{2}|V|^{2}\right)(t)+\frac{\delta^{2}}{2}|V|^{\frac{2 n}{n-2}}(t) .
$$

Then $\Psi^{\prime}(t) \equiv 0$, namely $\Psi(t)$ is a constant $K$. As a consequence it is easy to see that $v_{i},\left|v_{i}^{\prime}\right|$ are bounded. Indeed, if we suppose that there exists a sequence $t_{n} \rightarrow+\infty$ such that $\left|V\left(t_{n}\right)\right| \rightarrow+\infty$, we have

$$
\frac{1}{2}\left[\left(\frac{\left|V^{\prime}\left(t_{n}\right)\right|}{\left|V\left(t_{n}\right)\right|}\right)^{2}-\delta^{2}\right]+\frac{\delta^{2}}{2}\left|V\left(t_{n}\right)\right|^{\frac{4}{n-2}}=\frac{K}{\left|V\left(t_{n}\right)\right|^{2}}
$$


which is a contradiction. Similarly, we conclude that $\left|v_{i}^{\prime}\right|$ is bounded.

The first consequence of the radial symmetry of solutions is the fact that each coordinate $u_{i}$ satisfies the following dichotomy: either it is strictly positive, or it vanishes identically.

Lemma 2.3 Let $\mathcal{U}$ a nonnegative singular solution of the limit system (6). If there exist $y \in \mathbb{R}^{n} \backslash\{0\}$ such that $u_{i}(y)=0$, for some $i \in\{1,2\}$ then $u_{i} \equiv 0$.

Proof. We already know that our coordinates are radially symmetric. Suppose that there exist $y, z \in \mathbb{R}^{n} \backslash\{0\}$, with $|z|>|y|=r, u_{i}(y)=u_{i}(r)=0$ and $u_{i}(|z|)>0$. By the maximum principle of Chen and Lin in [4, we know that

$$
u_{i}(x) \geq \inf _{\partial B_{|z|}} u_{i}=u_{i}(|z|)>0, \quad \forall x \in B_{|z|}(0) \backslash\{0\}
$$

which contradicts the fact that $u_{i}(y)=0$. Hence, if there exists a point $y$ such that $u_{i}(y)=0$ then $u_{i}$ must be zero for all $z$ with $|z| \geq|y|$.

However, if we consider the change of variables of Fowler, we know that $v_{i}$ is a solution for the ODE system (2.6), and by the uniqueness of solutions to ODE systems, it would be identically zero.

In order to prove the classification result, we will need the following technical results

Lemma 2.4 Fix $i \in\{1,2\}$. If $v_{i}$ has a limit $C_{i}$ at $+\infty$ or $-\infty$, then $C_{i} \leq\left(\frac{n-2}{n}\right)^{\frac{n-2}{4}}$.

Proof. Assume $\lim _{t \rightarrow+\infty} v_{1}(t)=C_{1}$ where $C_{1}>0$. For each coordinate consider a sequence of translates $v_{i, k}(t)=v_{i}(t+k)$. By (2.6), up to passing to a subsequence, we may assume that $v_{1, k} \rightarrow C_{1}$ and $v_{2, k}^{2} \rightarrow w \geq 0$ uniformly in compacts with respect to the $C^{2}$ topology. Thus $-\delta^{2} C_{1}+\frac{n(n-2)}{4}\left(C_{1}^{2}+w\right)^{\frac{2}{n-2}} C_{1}=0$ which implies that $C_{1} \leq\left(\frac{n-2}{n}\right)^{\frac{n-2}{4}}$.

For each $i$ such that the coordinate $u_{i}$ is strictly positive, we define the auxiliary functions $f_{i}: \mathbb{R} \rightarrow \mathbb{R}$ by

$$
f_{i}(t):=-\frac{1}{2}\left|v_{i}^{\prime}(t)\right|^{2}+\frac{\delta^{2}}{2} v_{i}(t)^{2}-\frac{\delta^{2}}{2} v_{i}(t)^{\frac{2 n}{n-2}} .
$$

By (2.6), we have $f_{i}^{\prime}(t)=\frac{\delta^{2}}{2}\left(|V|^{\frac{4}{n-2}}(t)-v_{i}(t)^{\frac{4}{n-2}}\right) v_{i}(t) v_{i}^{\prime}(t)$. That is, the functions $f_{i}$ and $v_{i}$ have exactly the same monotonicity. This property is very important and will be used in the sequel. 


\section{Lemma 2.5}

$$
v_{i}(t)<1, \quad \forall t \in \mathbb{R} \text { and } i=1,2 .
$$

Proof. Suppose by contradiction that the result is not true. By the Lemma 2.4, there exists a local maximum point $t_{0} \in \mathbb{R}$ of $v_{1}$ such that $v_{1}\left(t_{0}\right) \geq 1$. This implies that

$$
f_{1}\left(t_{0}\right)=\frac{\delta^{2}}{2} v_{1}^{2}\left(t_{0}\right)-\frac{\delta^{2}}{2} v_{1}\left(t_{0}\right)^{\frac{2 n}{n-2}} \leq 0 .
$$

We claim that there exists $t_{1}>t_{0}$ such that $v_{1}^{\prime}(t)<0$ for $t \in\left(t_{0}, t_{1}\right)$ and $v_{1}^{\prime}\left(t_{1}\right)=0$. Otherwise it would follow from Lemma 2.4 that $v_{1} \downarrow C_{1} \leq((n-2) / n)^{\frac{n-2}{4}}$ and $f_{1}(t) \downarrow$ $-C<0$ as $t \uparrow+\infty$. Then we have that $v_{1}^{\prime}(t) \rightarrow 0$ as $t \uparrow+\infty$ and so

$$
0>-C=\frac{\delta^{2}}{2} C_{1}^{2}-\frac{\delta^{2}}{2} C_{1}^{\frac{2 n}{n-2}}>0
$$

a contradiction. Hence there exists such $t_{1}$ and it satisfies $v_{1}^{\prime \prime}\left(t_{1}\right) \geq 0$. On the other hand

$$
f_{1}\left(t_{1}\right)=\frac{\delta^{2}}{2} v_{1}\left(t_{1}\right)^{2}-\frac{\delta^{2}}{2} v_{1}\left(t_{1}\right)^{\frac{2 n}{n-2}}<f_{1}\left(t_{0}\right) \leq 0,
$$

namely $v_{1}\left(t_{1}\right)>1$ which implies from 2.6 that $v_{1}^{\prime \prime}\left(t_{1}\right)<0$, a contradiction.

Lemma $2.6 f_{i}(t)>0$ and so $\left|v_{i}^{\prime}(t)\right|<\delta v_{i}(t)$ for all $t \in \mathbb{R}$ and $i=1,2$.

Proof. Suppose $f_{1}(s) \leq 0$ for some $s \in \mathbb{R}$. If $f_{1}^{\prime}(s)=0$, then $v_{1}^{\prime}(s)=0$, which implies by 2.7 that $v_{1}(s) \geq 1$, a contradiction with Lemma 2.5. So we may assume, without loss of generality, that $f_{1}^{\prime}(s)>0$, namely $v_{1}^{\prime}(s)>0$. If $v_{1}^{\prime}(t)>0$ for all $t \leq s$, then $v_{1}(t) \downarrow C_{1} \leq\left(\frac{n-2}{n}\right)^{\frac{4}{n-2}}$ and $f_{1}(t) \downarrow-C<0$ as $t \downarrow-\infty$, and we get a contradiction as in the proof of Lemma 2.5. So there exists $t_{0}<s$ such that $v_{1}^{\prime}(t)>0$ for $t \in\left(t_{0}, s\right]$ and $v_{1}^{\prime}\left(t_{0}\right)=0$. Similarly we get $v_{1}\left(t_{0}\right)>1$, a contradiction with Lemma 2.5 .

\subsection{Removable singularity result}

We now turn to a discussion of the existence and specific form of a family of homological integral invariants of solutions of system (1). These invariants were discovered in their simplest form by Pohozaev and was later put into a general Riemannian setting in the work of R. Schoen [19]. As we are working in a ball in the euclidian space, we can use a simple definition. 
For $r>0$, define the Pohozaev integral

$$
P(r, \mathcal{U})=\int_{\partial B_{r}}\left(\frac{n-2}{2}\left\langle\mathcal{U}, \frac{\partial \mathcal{U}}{\partial \nu}\right\rangle-\frac{r}{2}|\nabla \mathcal{U}|^{2}+r\left|\frac{\partial \mathcal{U}}{\partial \nu}\right|^{2}+r \frac{(n-2)^{2}}{8}|\mathcal{U}|^{\frac{2 n}{n-2}}\right) d \sigma
$$

where $\nu$ is the unit outer normal of $\partial B_{r}$. Multiplying each equation of (6) by $x \cdot \nabla u_{i}$ and integrating over $B_{r} \backslash B_{s}$ we verify for all $r, s>0$ that

Lemma 2.7 (Pohozaev Identity) For $0<s \leq r$, we have

$$
P(r, \mathcal{U})=P(s, \mathcal{U})
$$

Proof. Adding and subtracting $\Delta u_{i}$ in each system equation, we have

$$
\Delta u_{i}+\frac{n(n-2)}{4}|\mathcal{U}|^{\frac{4}{n-2}} u_{i}=0
$$

in the punctured ball $\Omega=B_{1}^{n}(0) \backslash\{0\}$. Mutiplying both sides by $x \cdot \nabla u_{i}$ and integrating over $B_{r} \backslash B_{s}$ we have

$$
-\int_{B_{r} \backslash B_{s}} x \cdot \nabla u_{i}\left(\Delta u_{i}+\frac{n(n-2)}{4}|\mathcal{U}|^{\frac{4}{n-2}} u_{i}\right)=0
$$

First using integration by parts we get

$$
\begin{aligned}
& \int_{B_{r} \backslash B_{s}} x \cdot \nabla u_{i} \Delta u_{i}=\frac{n-2}{2} \int_{B_{r} \backslash B_{s}}\left|\nabla u_{i}\right|^{2}-\frac{r}{2} \int_{\partial B_{r}}\left|\nabla u_{i}\right|^{2}+\frac{s}{2} \int_{\partial B_{s}}\left|\nabla u_{i}\right|^{2} \\
& +r \int_{\partial B_{r}}\left|\frac{\partial u_{i}}{\partial \nu}\right|^{2}-s \int_{\partial B_{s}}\left|\frac{\partial u_{i}}{\partial \nu}\right|^{2} .
\end{aligned}
$$

On the other hand, multiplying each equation for $u_{i}$ and integrating over $B_{r} \backslash B_{s}$ we have

$$
\begin{aligned}
& 0=-\int_{B_{r} \backslash B_{s}} u_{i}\left(\Delta u_{i}+\frac{n(n-2)}{4}|\mathcal{U}|^{\frac{4}{n-2}} u_{i}\right)= \\
& \int_{B_{r} \backslash B_{s}}\left|\nabla u_{i}\right|^{2}-\int_{\partial B_{r}} u_{i} \frac{\partial u_{i}}{\partial \nu}+\int_{\partial B_{s}} u_{i} \frac{\partial u_{i}}{\partial \nu}-\frac{n(n-2)}{4} \int_{B_{r} \backslash B_{s}}|\mathcal{U}|^{\frac{4}{n-2}} u_{i}^{2}
\end{aligned}
$$

which implies that

$$
\int_{B_{r} \backslash B_{s}}\left|\nabla u_{i}\right|^{2}=\int_{\partial B_{r}} u_{i} \frac{\partial u_{i}}{\partial \nu}-\int_{\partial B_{s}} u_{i} \frac{\partial u_{i}}{\partial \nu}+\frac{n(n-2)}{4} \int_{B_{r} \backslash B_{s}}|\mathcal{U}|^{\frac{4}{n-2}} u_{i}^{2}
$$

Replacing 4.27) in 2.10, we conclude that

$$
\begin{aligned}
& \int_{\partial B_{r}}\left[\frac{n-2}{2} u_{i} \frac{\partial u_{i}}{\partial \nu}-\frac{r}{2}\left|\nabla u_{i}\right|^{2}+r\left|\frac{\partial u_{i}}{\partial \nu}\right|^{2}\right]-\int_{\partial B_{s}}\left[\frac{n-2}{2} u_{i} \frac{\partial u_{i}}{\partial \nu}-\frac{s}{2}\left|\nabla u_{i}\right|^{2}+s\left|\frac{\partial u_{i}}{\partial \nu}\right|^{2}\right] \\
& =\quad-\frac{n(n-2)^{2}}{8} \int_{B_{r} \backslash B_{s}}|\mathcal{U}|^{\frac{2 n}{n-2}}-\frac{n(n-2)}{4} \int_{B_{r} \backslash B_{s}}\left(x \cdot \nabla u_{i}|\mathcal{U}|^{\frac{4}{n-2}} u_{i}\right) .
\end{aligned}
$$


Note also that, using $\frac{n-2}{2 n} \partial_{k}\left(|\mathcal{U}|^{\frac{2 n}{n-2}}\right)=\sum_{i}|\mathcal{U}|^{\frac{4}{n-2}} u_{i} \partial_{k} u_{i}$, we obtain

$$
\begin{gathered}
-\sum_{i} \int_{B_{r} \backslash B_{s}} x \cdot \nabla u_{i}|\mathcal{U}|^{\frac{4}{n-2}} u_{i}=\frac{n-2}{2} \int_{B_{r} \backslash B_{s}}|\mathcal{U}|^{\frac{2 n}{n-2}}-r \frac{n-2}{2 n} \int_{\partial B_{r}}|\mathcal{U}|^{\frac{2 n}{n-2}} \\
+s \frac{n-2}{2 n} \int_{\partial B_{s}}|\mathcal{U}|^{\frac{2 n}{n-2}}
\end{gathered}
$$

which finishes the proof.

From the above result we know that $P(r, \mathcal{U})$ is a constant independent of $r$ that we denote by $P(\mathcal{U})$. We will prove that

Theorem 2.8 Let $\mathcal{U}$ be a positive solution of (6). Then $P(\mathcal{U}) \leq 0$. Moreover, $P(\mathcal{U})=$ 0 if and only if each coordinate $u_{i}$ is smooth at 0.

Proof. Our strategy to prove the result about removable singularity will be suppose that $P(\mathcal{U}) \geq 0$, to prove that necessarily we have $P(\mathcal{U})=0$ and then that all the components of the solution are smooth.

Suppose that $P(\mathcal{U}) \geq 0$. We want to prove that $P(\mathcal{U})=0$ and $u_{i} \in C^{2}\left(\mathbb{R}^{n}\right)$. Recall that $K \geq 0$. Then we have,

$$
0<\sum_{i=1}^{2} f_{i}(t)=\frac{\delta^{2}}{2}|V|^{\frac{2 n}{n-2}}-\sum_{i=1}^{2} \frac{\delta^{2}}{2} v_{i}(t)^{\frac{2 n}{n-2}}-K \leq \frac{\delta^{2}}{2}|V|^{\frac{2 n}{n-2}}-\sum_{i=1}^{2} \frac{\delta^{2}}{2} v_{i}(t)^{\frac{2 n}{n-2}}
$$

According to the Lemma 2.3, we can consider two different situations. If one of the coordinates for the solution vanishes, we have a contradiction from the above expression. Otherwise, Lemma 2.4 implies the existence of $s \in \mathbb{R}$ such that $v_{1}^{\prime}(s)=0$. Let $s \in \mathbb{R}$ be any a point such that $v_{1}^{\prime}(s)=0$. Then, by Lemma 2.6 and 2.12),

$$
f_{1}(s)=\frac{\delta^{2}}{2} v_{1}(s)^{2}-\frac{\delta^{2}}{2} v_{i}(s)^{\frac{2 n}{n-2}}<\frac{\delta^{2}}{2}|V|^{\frac{2 n}{n-2}}-\sum_{i=1}^{2} \frac{\delta^{2}}{2} v_{i}(s)^{\frac{2 n}{n-2}} .
$$

Note that in the right side of the equation, we will only have terms that are multiples of $v_{1}(s)^{2}$. So dividing everything by $v_{1}(s)^{2}$ and estimating the terms that remain, we obtain by a direct computation

$$
\frac{|V|^{\frac{2 n}{n-2}}-\sum_{i=1}^{2} v_{i}(s)^{\frac{2 n}{n-2}}}{v_{i}(s)^{2}}+v_{i}(s)^{\frac{4}{n-2}} \leq C|V|^{\frac{4}{n-2}},
$$

where $C=\frac{2^{*}}{2}$. Then

$$
\delta^{2}<\frac{n(n-2)}{4}|V|^{\frac{4}{n-2}}
$$


This inequality implies that

$$
v_{1}^{\prime \prime}(s)=\left(\delta^{2}-\frac{n(n-2)}{4}|V|^{\frac{4}{n-2}}\right) v_{1}(s)<0 .
$$

That is, $s$ must be a local maximum point of $v_{1}$, and $v_{1}$ has no local minimum points. Therefore, $v_{1}$ has a unique maximum point $t_{1}, v_{1}^{\prime}(t)>0$ for $t<t_{1}$ and $v_{1}^{\prime}(t)<0$ for $t>t_{1}$. Similarly, $v_{2}$ has a unique maximum point $t_{2}, v_{2}^{\prime}(t)>0$ for $t<t_{2}$. Lemma 2.4 gives that $v_{2} \downarrow 0, \forall i$ as $t \uparrow+\infty$. This, together with Lemma 2.6, yields $K=0$, namely $P(\mathcal{U})=0$. Let $v=\sum_{i=1}^{2} v_{i}$ and $y(t)=v^{\prime}(t)+\delta v(t)$ then $v^{\prime}(t)<0$ for all $t>\max _{1 \leq i \leq 2} t_{i}$ and

$$
y^{\prime}-\delta y=v^{\prime \prime}-\delta^{2} v=-\frac{n(n-2)}{4}|V|^{\frac{4}{n-2}} v \geq-C v^{\frac{n+2}{n-2}} .
$$

Since $y>0$ is bounded, it is easy to prove that

$$
v^{\prime}(t)+\delta v(t)=y(t) \leq \frac{C}{\delta} v^{\frac{n+2}{n-2}}, \quad \forall t>\max _{1 \leq i \leq 2} t_{i}
$$

and then $\left(e^{\delta t} v(t)\right)^{2-2^{*}}-\frac{C}{\delta} e^{\left(2-2^{*}\right) \delta t}$ is strictly increasing for $t>\max _{1 \leq i \leq 2} t_{i}$. Combining this with $v(t) \downarrow 0$ as $t \uparrow+\infty$ we get $e^{\delta t} v(t) \leq C$ uniformly for $t>0$ large. That is $\sum_{i=1}^{2} u_{i}(r) \leq C$ uniformly for $r>0$ small. Therefore, for standard elliptic regularity theory, $u_{i} \in C^{2}\left(\mathbb{R}^{n}\right), \forall i$, which ends the proof.

\subsection{Classification Result}

In the beggining of this chapter, we mentioned that the solutions of our limit system play a similar role to Fowler's solutions in the singular Yamabe problem.

On the other hand, O. Druet, E. Hebey and J. Vétois in [6], [7] proved the following characterization result for entire solutions in $\mathbb{R}^{n}$ for the limit system

Proposition 2.9 ([7]) Let $\mathcal{U}$ be a nonnegative $C^{2}$-solution of

$$
\Delta u_{i}+|\mathcal{U}|^{\frac{4}{n-2}} u_{i}=0 \quad \text { in } \quad \mathbb{R}^{n} .
$$

Then there exist $a \in \mathbb{R}^{n}, \mu>0$ and $\Lambda \in \mathbb{S}_{+}^{1}$, such that

$$
\mathcal{U}(x)=\left(\frac{\mu}{\mu^{2}+\frac{|x-a|^{2}}{n(n-2)}}\right)^{\frac{n-2}{2}} \Lambda,
$$

for all $x \in \mathbb{R}^{n}$, where $\mathbb{S}_{+}^{1}$ consists of the elements $\left(\Lambda_{1}, \Lambda_{2}\right)$ in the unit sphere such that each coordinate is nonnegative. 
Inspired by their result, we may ask wheter a similar description holds in the singular case. Indeed, we will prove that every nonnegative singular solution of (6) it is a Fowler solution times a vector in the unit sphere with nonnegative coordinates.

Theorem 2.10 Suppose that $\mathcal{U}_{0}$ is a nonnegative singular solution for (6). The there exists a $\Lambda \in \mathbb{S}_{+}^{1}$ and $u_{0}$ a Fowler solution such that

$$
\mathcal{U}_{0}=u_{0} \Lambda
$$

where $\mathbb{S}_{+}^{1}=\left\{x \in \mathbb{S}^{1}: x_{i} \geq 0\right\}$.

Proof. We will prove the result in the case where $v_{1}$ and $v_{2}$ are positive solutions since otherwise the result follows directly from Lemma 2.3

Let $\mathcal{V}=\left(v_{1}, v_{2}\right)$ be the solution for (2.6) obtained from $\mathcal{U}_{0}$ after the change of variables of Fowler. We know that it satisfies

$$
v_{i}^{\prime \prime}-\delta^{2} v_{i}+\frac{n(n-2)}{4}|\mathcal{V}|^{\frac{4}{n-2}} v_{i}=0, \quad t \in \mathbb{R} .
$$

From this, we can note that

$$
v_{1}^{\prime \prime}(t) v_{2}(t)=v_{1}(t) v_{2}^{\prime \prime}(t)
$$

which implies

$$
v_{1}^{\prime}(t) v_{2}(t)-v_{1}(t) v_{2}^{\prime}(t)=c
$$

for all $t \in \mathbb{R}$, where $c$ is a constant.

Suppose that $c \neq 0$. Whitout loss of generality we can assume that $c>0$. Then by Lemma 2.4 we obtain

$$
\left(\frac{v_{1}}{v_{2}}\right)^{\prime}(t)=\frac{c}{v_{2}^{2}(t)} \geq c
$$

which is a contradiction, since by (2.14) the quocient would assume negative values. Therefore we conclude that

$$
\left(\frac{v_{1}}{v_{2}}\right)^{\prime}(t)=0
$$

and so $v_{1} / v_{2}=\eta$ is constant. It follows that

$$
\mathcal{V}=\left(v_{1}, v_{2}\right)=\left(\eta v_{2}, v_{2}\right)=(\eta, 1) v_{2}=\sqrt{1+\eta^{2}} v_{2}\left(\frac{\eta}{\sqrt{1+\eta^{2}}}, \frac{1}{\sqrt{1+\eta^{2}}}\right)
$$

and a direct computation shows that $v_{0}=\sqrt{1+\eta^{2}} v_{2}$ is a Fowler solution. 
Although the proof above is quite simple, the result will be extremely important in the study of the Jacobi fields since will simplify the linearized system, and it also allows us to use all of the properties from Fowler solutions that we described previously. Inspired by this result, we will call the solutions of the system (6) of Fowler-type solutions or Delaunay-type solutions.

Let us mention some important consequences of the theorem. We observed in the introduction of this section that the definition of singular solution thus not imply that both solutions blows up at the origin but, as consequence of Theorem (2.10), we can see that this is exactly what happens when both coordinates are strictly positive.

Corollary 2.11 Suppose that $\mathcal{U}=\left(u_{1}, u_{2}\right)$ is a solution for (6), such that both coordinates are strictly positive. Then $\lim _{|x| \rightarrow 0} u_{i}(x)=+\infty$, for $i=1,2$.

Our second direct consequence will be the following asymptotic behavior

Corollary 2.12 If $\mathcal{U}$ is a nonnegative singular solution to the system (6), then $\mathcal{U}$ is an admissible solution, that is, there exists positive constants $c_{1}, c_{2}$ such that

$$
c_{1}|x|^{\frac{2-n}{2}} \leq|\mathcal{U}|(x) \leq c_{2}|x|^{\frac{2-n}{2}} .
$$

Besides note that by the classification result, there exists a Fowler solution $u_{0}$ and an unit vector $\Lambda=\left(\Lambda_{1}, \Lambda_{2}\right)$ with nonnegative coordinates, such that $\mathcal{U}=u_{0} \Lambda$. Consequently $P(\mathcal{U})$ coincides with the Pohozaev invariant of $u_{0}$ as defined in 3 which implies that the removable singularity result $(2.8)$ proved in one of the previous sections follow as a direct consequence of Theorem 2.10.

\subsection{Jacobi Fields for the Limit System}

Carrying out the study intended in the following chapters, in this last section we will study what we call the Jacobi fields for the limit system.

In the study of the asymptotic behavior of the singular Yamabe problem in the punctured ball for flat and non flat case, N. Korevaar et al. in [11] and F. Marques [13] respectively, used the previously known growth properties of Jacobi fields as a tool to obtain information about the singular problem. 
In order to do a similar analysis we are going to linearize solutions of system around the Fowler-type solutions that we characterized in the previous subsection. Consider the operator in cylindrical coordinates $H(\mathcal{V})=\left(H^{1}(\mathcal{V}), H^{2}(\mathcal{V})\right)$ where

$$
H^{i}(\mathcal{V})=\partial_{t}^{2} v_{i}+\Delta_{\mathbb{S} n-1} v_{i}-\frac{(n-2)^{2}}{4} v_{i}+\frac{n(n-2)}{4}|\mathcal{V}|^{\frac{4}{n-2}} v_{i}
$$

When we linearize this operator on a solution $\mathcal{V}_{0}=\left(v_{0,1}, v_{0,2}\right)$, we obtain that the linearized operator is given by $\mathcal{L}(\phi)=\left(\mathcal{L}^{1}(\phi), \mathcal{L}^{2}(\phi)\right)$ where

$$
\begin{aligned}
\mathcal{L}^{i}(\phi) & =\left.\frac{\partial}{\partial t}\right|_{t=0} H^{i}\left(\mathcal{V}_{0}+t \phi\right) \\
& =\partial_{t}^{2} \phi_{i}+\Delta_{\mathbb{S} n-1} \phi_{i}-\frac{(n-2)^{2}}{4} \phi_{i}+\frac{n(n-2)}{4}\left(\frac{4}{n-2}\left|\mathcal{V}_{0}\right|^{2^{*}-4}\left\langle\mathcal{V}_{0}, \phi\right\rangle v_{0, i}+\left|\mathcal{V}_{0}\right|^{\frac{4}{n-2}} \phi_{i}\right) .
\end{aligned}
$$

However from the characterization result obtained in Theorem 2.10, we know that there exist a Fowler solution $v_{\varepsilon}$ and a vector in the unit sphere $\Lambda=\left(\Lambda_{1}, \Lambda_{2}\right)$ with positive coordinates such that $V_{0}=v_{\varepsilon} \Lambda$. Using this fact, our linearized system can be simplified and written as follows

$$
\mathcal{L}_{\varepsilon}^{i}(\phi)=\partial_{t}^{2} \phi_{i}+\Delta_{\mathbb{S}^{n-1}} \phi_{i}-\frac{(n-2)^{2}}{4} \phi_{i}+n \Lambda_{i}\langle\Lambda, \phi\rangle v_{\varepsilon}^{\frac{4}{n-2}}+\frac{n(n+2)}{4} v_{\varepsilon}^{\frac{4}{n-2}} \phi_{i} .
$$

We will focus now in the study of the Jacobi fields for the system, that is, solutions of the linear ODE system $L_{\varepsilon}(\mathcal{V})=0$.

Similarly to the case of a single equation, we can consider the spectral decomposition of the operator $\Delta_{\mathbb{S}^{n-1}}$ writing $\left\{\lambda_{k}, \mathcal{X}_{k}(\theta)\right\}$ for its eingendata and if $\phi=\left(\phi_{1}, \phi_{2}\right)$ is a solution of the system $\mathcal{L}_{\varepsilon}^{i}(\phi)=0$, then for each coordinate

$$
\phi_{i}(t)=\sum v_{i, k}(t) \mathcal{X}_{k}(\theta)
$$

where $v_{i, k}$ satisfies

$$
\mathcal{L}_{\varepsilon, k} \phi_{i, k}+n \Lambda_{i}\left\langle\Lambda, \phi_{k}\right\rangle v_{\varepsilon}^{\frac{4}{n-2}}+\frac{n(n-2)}{4} v_{\varepsilon}^{\frac{4}{n-2}} \phi_{i}=0
$$

and $\mathcal{L}_{\varepsilon, k}=\partial_{t}^{2}-\left(\lambda_{k}+\frac{(n-2)^{2}}{4}\right)$.

We are going to obtain the Jacobi fields for the first eingenvalue and we will see that the construction for higher eingenvalues is similar.

In the case of only one equation we saw that the two linearly independent fields were obtained as derivatives of one-parameter families of solutions from 2.16). However, as we are working with an ODE linear system with two equations, we expect to obtain four linearly independent Jacobi fields. 
For the system, we can consider three natural one-parameter families of solutions. Indeed, similarly to 1.8 , let

$$
T \rightarrow v_{\varepsilon}(t+T) \Lambda \quad \text { and } \quad \varepsilon \rightarrow v_{\varepsilon} \Lambda .
$$

The derivatives of these families provide us the solutions $\phi_{\varepsilon, 0}^{1}=\psi_{\varepsilon, 0}^{+} \Lambda$ and $\phi_{\varepsilon, 0}^{2}=$ $\psi_{\varepsilon, 0}^{-} \Lambda$, where $\psi_{\varepsilon, 0}^{ \pm}$are the previously described linearly independents Jacobi fields for one equation. Consequently our solutions are also linearly independent and have the same growth of the original fields.

Observe also that is possible to build another one-parameter family of solutions to (2.17). In fact, let $\Lambda(\alpha)=\left(\cos \left(\alpha+\alpha_{0}\right), \sin \left(\alpha+\alpha_{0}\right)\right)$ with $\Lambda(0)=\left(\cos \alpha_{0}, \sin \alpha_{0}\right)=\Lambda$ be a path in the sphere and consider the family

$$
\alpha \rightarrow v_{\varepsilon} \Lambda(\alpha)
$$

If we differentiate this family with respect to the parameter we get a third the Jacobi field $\phi_{\varepsilon, 0}^{3}=v_{\varepsilon} \bar{\Lambda}$, where $\bar{\Lambda}=\left(-\Lambda_{2}, \Lambda_{1}\right)$. Note as the vectors $\Lambda$ and $\bar{\Lambda}$ are orthogonal we automatically have that this field is linearly independent to the other two that we have already built. Moreover $\phi_{\varepsilon, 0}^{3}$ is also a periodic field.

Unfortunately, we are only able to construct the last Jacobi field in an indirect way. Observe first that $v_{\varepsilon}$ is a solution for the $\operatorname{ODE} \bar{L}_{\varepsilon, 0} v_{\varepsilon}=0$, where

$$
\bar{L}_{\varepsilon, 0}=\partial_{t}^{2}-\frac{(n-2)^{2}}{4}+\frac{n(n-2)}{4} v_{\varepsilon}^{\frac{4}{n-2}} \text {. }
$$

We are writing the operator from Fowler equation in this manner intentionally. As this operator is linear we know that there exists a solution $\phi_{\varepsilon, 0}^{-}$to 2.18 which is linearly independent to $\phi_{\varepsilon, 0}^{+}:=v_{\varepsilon}$. The last Jacobi field will be given by $\phi_{0}^{4}=\phi_{\varepsilon, 0}^{-} \bar{\Lambda}$. Also as $\bar{L}_{\varepsilon, 0}<L_{\varepsilon, 0}$, using standard methods of comparison theory for ODE's, we know that the growth of this field is at most linear.

Therefore we constructed four linearly independent Jacobi fields for the system with respect to the first eingenvalue.

Based on the construction above, the Jacobi fields for the higher eingenvalues $\lambda_{k}$ will be given by $\phi_{k}^{1}=\psi_{\varepsilon, k}^{+} \Lambda, \phi_{k}^{2}=\psi_{\varepsilon, k}^{-} \Lambda$, where $\psi_{\varepsilon, k}^{ \pm}$are the original solutions constructed for one equation which we alredy know the growth. The remaining two solutions are $\phi_{k}^{3}=\phi_{\varepsilon, k}^{+} \bar{\Lambda}, \phi_{k}^{4}=\phi_{\varepsilon, k}^{-} \bar{\Lambda}$, where $\phi_{\varepsilon, k}^{ \pm}$are solutions of

$$
\bar{L}_{\varepsilon, k} v=\partial_{t}^{2} v+\left(\frac{n(n-2)}{4} v_{\varepsilon}^{\frac{4}{n-2}}-\frac{(n-2)^{2}}{4}-\lambda_{k}\right) v=0 .
$$


We know that the zero order term of the above operator is negative when $k>0$ since $v_{\varepsilon}<1$ and $\lambda_{k} \geq n-1$ for such $k$. This implies that $\bar{L}_{\varepsilon, k}$ satisfies the maximum principle, so we are able to determine the growth of these fields, concluding our analysis. 


\section{Chapter 3}

\section{Coupled elliptic system in the punctured ball}

In this chapter we will study asymptotics of singular nonnegative solutions to the following system

$$
\Delta u_{i}+\frac{n(n-2)}{4}|\mathcal{U}|^{\frac{4}{n-2}} u_{i}=0
$$

in the punctured ball $B_{1}(0) \backslash\{0\}$, where $|\mathcal{U}|^{2}=u_{1}^{2}+u_{2}^{2}$ and $\Delta$ denotes the euclidian laplacian. This system is the natural generalization of the singular Yamabe problem in the flat case.

It will be convenient to consider $v_{i}$ obtained from $u_{i}$ by the change of variables of Fowler in order to work with the equivalent system

$$
L_{c y l} v_{i}+\frac{n(n-2)}{4}|\mathcal{V}|^{\frac{4}{n-2}} v_{i}=0
$$

where $L_{c y l}=\partial_{t}^{2}+\Delta_{\mathbb{S}^{n-1}}-\frac{(n-2)^{2}}{4}$ is the cylindrical laplacian.

\subsection{Upper bound}

In this section our main purpose is to prove an upper bound for a solution $\mathcal{U}$ of (3.1) which is singular at the origin. Such a result will be obtained as a direct consequence of an inequality for positive supersolutions of (3.1) that are smooth in an open set $\Omega \subset B_{1}(0)$. The complement $\Gamma=B_{1}(0) \backslash \Omega$ is then a relatively closed set in the unit ball. 
Remember that a positive solution $\mathcal{U}$ is a weak supersolution of $(3.1)$ if it lies in $L^{(n+2) /(n-2)}\left(B_{1}(0)\right)^{2}$ and if it satisfies

$$
\Delta u_{i}+\frac{n(n-2)}{4}|\mathcal{U}|^{\frac{4}{n-2}} u_{i} \leq 0
$$

in the distributional sense on $B_{1}(0)$.

We know that any solution $\mathcal{U}$ which lies locally in $L^{\frac{n+2}{n-2}}\left(B_{1}(0)\right)^{2}$ and tends uniformly to infinity near $\Gamma$ may be extended to a weak supersolution in $B_{1}(0)$. Indeed, for any large constant $L$ if we define $\mathcal{U}_{L}$ whose coordinate functions are given by $u_{i, L}=\min \left\{u_{i}, L\right\}$ then

$$
\Delta u_{i, L} \leq-\frac{n(n-2)}{4}\left|\mathcal{U}_{L}\right|^{\frac{4}{n-2}} u_{i, L} \chi|\mathcal{U}| \leq L
$$

where $\chi_{E}$ denotes the characteristic function of a set $E$. Applying the dominated convergence theorem we conclude then that $\mathcal{U}$ defines a weak supersolution.

However, if we consider a solution $\mathcal{U}$ of the system (3.1) which is smooth on an arbitrary open subset $\Omega$, we can not conclude imediatly that the solution lies locally in $L^{\frac{n+2}{n-2}}\left(B_{1}(0)\right)^{2}$. In order to get this we will assume that the relatively closed set $\Gamma=B_{1}(0) \backslash \Omega$ is thin.

More precisely, let $\Gamma \subset B_{1}(0)$ be a relatively closed set of Lebesgue measure zero. We will say that $\Gamma \subset B_{1}(0)$ is a thin set if there exists a sequence $\phi_{k}$ of smooth functions in $B_{1}(0)$, such that

$$
\left\{\begin{array}{l}
\phi_{k} \equiv 0 \quad \text { in a neighborhood of } \Gamma \\
0 \leq \phi_{k} \leq 1 \\
\phi_{k} \text { converges to } 1 \text { in } B^{n} \backslash \Gamma
\end{array}\right.
$$

and

$$
\lim _{k \rightarrow \infty} \int_{B_{r}(0)}\left(\left|\Delta \phi_{k}\right|^{\frac{n+2}{4}}+\left|\nabla \phi_{k}\right|^{\frac{n+2}{2}}\right) d x=0 .
$$

for any $r<1$. Observe that a submanifold of dimension at most $(n-2) / 2$ is thin and consequently, a point is thin in any dimension $n \geq 2$.

Lemma 3.1 Suppose $\mathcal{U}$ is a solution of (3.1) on $\Omega=B_{1}(0) \backslash \Gamma$ where $\Gamma$ is a relatively closed thin set. Then $\mathcal{U}$ lies locally in $L^{\frac{n+2}{n-2}}\left(B_{1}(0)\right)^{2}$, and defines a weak solution of (3.1) on $B_{1}(0)$. 
Proof. Let $\xi$ be a nonnegative smooth function compactly supported in $B_{1}(0) \backslash \Gamma$. Multiplying the system 3.1 by $\xi^{\frac{n+2}{2}}$ and integrating by parts we have

$$
-\int u_{i} \Delta\left(\xi^{\frac{n+2}{2}}\right) d x=\frac{n(n-2)}{4} \int|\mathcal{U}|^{\frac{4}{n-2}} u_{i} \xi^{\frac{n+2}{2}}
$$

for each coordinate $u_{i}$, which taking the sum in $i$ implies the bound

$$
\int|\mathcal{U}|^{\frac{n+2}{n-2}} \xi^{\frac{n+2}{2}} d x \leq c \int \xi^{\frac{n-2}{2}} u\left(\xi|\Delta \xi|+|\nabla \xi|^{2}\right) d x
$$

where $u=u_{1}+u_{2}$. Applying Hölder's inequality we obtain

$$
\int\left(\xi^{\frac{n-2}{2}}|\mathcal{U}|\right)^{\frac{n+2}{n-2}} d x \leq c \int\left(\xi|\Delta \xi|+|\nabla \xi|^{2}\right)^{\frac{n+2}{4}} d x .
$$

Choose now $\xi=\psi \phi_{k}$, where $\psi$ is a function which is equal to 1 in $B_{r}(0)$ for some $r<1$ and equal to zero outside $B_{r_{1}}(0)$, for some $r<r_{1}$. Then the following estimate holds

$$
\left(\xi|\Delta \xi|+|\nabla \xi|^{2}\right)^{\frac{n+2}{4}} \leq c\left(1+\left|\Delta \phi_{k}\right|^{\frac{n+2}{4}}+\left|\nabla \phi_{k}\right|^{\frac{n+2}{2}}\right) .
$$

Replacing this estimate in 3.3 , we have that

$$
\int|\mathcal{U}|^{\frac{n+2}{n-2}} \xi^{\frac{n+2}{2}} d x \leq c \int\left(1+\left|\Delta \phi_{k}\right|^{\frac{n+2}{4}}+\left|\nabla \phi_{k}\right|^{\frac{n+2}{2}}\right) d x \leq c
$$

proving that each $u_{i} \in L^{\frac{n+2}{n-2}}\left(B_{r}(0)\right)$ for any $r<1$, and hence $u_{i} \in L_{l o c}^{\frac{n+2}{n-2}}\left(B_{1}(0)\right)$.

We will prove now that $\mathcal{U}$ is a weak solution for the problem 3.1). In order to do this, given any smooth funtion $\xi$ with compact support in $B^{n}$ we need to show that

$$
\int_{B_{1}(0)}\left(u_{i} \Delta(\xi)+\frac{n(n-2)}{4} \xi|\mathcal{U}|^{\frac{4}{n-2}} u_{i}\right) d x=0 .
$$

Indeed, if we multiply the system (3.1) by $\xi \phi_{k}$ and integrate by parts, we obtain

$$
\int_{B_{1}(0)}\left(u_{i} \Delta\left(\xi \phi_{k}\right)+\frac{n(n-2)}{4} \xi \phi_{k}|\mathcal{U}|^{\frac{4}{n-2}} u_{i}\right) d x=0 .
$$

We may calculate

$$
\Delta\left(\xi \phi_{k}\right)=\xi \Delta \phi_{k}+2\left\langle\nabla \xi, \nabla \phi_{k}\right\rangle+\phi_{k} \Delta \xi
$$

By Hölder's inequality it follows that

$$
\left|\int u_{i} \xi \Delta\left(\phi_{k}\right) d x\right| \leq\left\|u_{i} \xi\right\|_{L^{(n+2) /(n-2)}}\left\|\Delta\left(\phi_{k}\right)\right\|_{L^{(n+2) / 4}(s u p \xi)}
$$


and the right-hand side tends to zero as $k$ tends to infinity. Similarly

$$
\left|\int u_{i}\left\langle\nabla \xi, \nabla \phi_{k}\right\rangle d x\right| \leq\left\|u_{i}|\nabla \xi|\right\|_{L^{(n+2) /(n-2)}}\left\|\nabla \phi_{k}\right\|_{L^{(n+2) / 4}(\sup \xi)}
$$

and the right-hand side converges to zero. Therefore applying the dominated convergence theorem and letting $k \rightarrow \infty$ we conclude the result.

Another key ingredient in order to obtain the upper bound is the existence of spherical solutions on $\mathbb{R}^{n}$. Remember that for any $\mu>0$ and $x_{0} \in \mathbb{R}^{n}$, the spherical solution of dilatation $\mu$ and center $x_{0}$ is given by

$$
u_{\mu, x_{0}}(x)=\left(\frac{2 \mu}{1+\mu^{2}\left|x-x_{0}\right|^{2}}\right)^{\frac{n-2}{2}} .
$$

As mentioned in the introduction of this thesis, O. Druet, E. Hebey and J. Vetóis [7] proved that positive solutions of (3.1) in $\mathbb{R}^{n}$ are given by a spherical solution times a vector $\Lambda$ in the unit sphere with positive coordinates. We can now state and prove the upper bound estimate.

Theorem 3.2 Let $\mathcal{U}$ be a positive supersolution of (3.1) which is a smooth solution on an open set $\Omega \subset B_{1}(0)$. Then there exists a positive constant $c$ such that

$$
|\mathcal{U}|(x) \leq c d(x, \Gamma)^{\frac{2-n}{2}}\left(\inf _{\partial B_{3 / 4}(0)}|\mathcal{U}|\right)^{-1}
$$

for all $x \in \Omega \cap B_{1 / 2}(0)$.

Proof. Denote by $\Gamma_{\varepsilon}$ the neighborhood with radius $\varepsilon$ about $\Gamma$. For $x \in B_{5 / 8}(0)$, set $U_{\varepsilon}(x)=d_{\varepsilon}(x)^{\frac{n-2}{2}}|\mathcal{U}|(x)$ where $d_{\varepsilon}(x)=\min \left\{d\left(x, \Gamma_{\varepsilon}\right), \frac{5}{8}-|x|\right\}$. In order to prove the Theorem it is enough to show that

$$
\sup _{B_{5 / 8}(0)} U_{\varepsilon} \leq c\left(\inf _{\partial B_{3 / 4}(0)}|\mathcal{U}|\right)^{-1}
$$

for some constant $c$ not depending on $\varepsilon$.

Let $\eta=\left|\mathcal{U}\left(x_{0}\right)\right|^{-\frac{2}{n-2}}$, where $x_{0}$ is a maximum point of $U_{\varepsilon}$ and consider the rescaled functions

$$
w_{i, \eta}(x)=\eta^{\frac{n-2}{2}} u_{i}\left(x_{0}+\eta x\right)
$$

The first step to prove our result is the following claim. 
Claim 1: Let $R>0$ and $\delta \in\left(0, \frac{1}{2}\right]$ be constants. There exists a constant $c_{1}$ depending only of $n, R$ and $\delta$ such that if $M_{\varepsilon} \equiv \sup _{B\left(0, \frac{5}{8}\right)} U_{\varepsilon}(x) \geq c_{1}$ then

$$
\left\|u_{\mu, y_{0}} \Lambda_{i}-w_{i, \eta}\right\|_{C^{2}\left(B_{R}(0)\right)}<\delta
$$

where $u_{\mu, y_{0}}$ is the spherical solution defined in with $\left|y_{0}\right| \leq c_{2}$ and $1 / c_{2} \leq \mu \leq c_{2}$, and $\Lambda=\left(\Lambda_{1}, \Lambda_{2}\right)$ is a vector in the unit sphere with nonegative coordinates.

Suppose by contradiction that this claim is not true. Then there would exist a sequence of maximum points $x_{k} \in B_{5 / 8}(0)$ of $U_{\varepsilon}$ such that $U_{\varepsilon}\left(x_{k}\right)$ converges to infinity and (3.6) does not hold for any $\mu>0$ and any $y_{0} \in R^{n}$. Let

$$
w_{i, k}(x)=\eta_{k}^{\frac{n-2}{2}} u_{i}\left(x_{k}+\eta_{k} x\right)
$$

for each coordinate, where $\eta_{k}=\left|\mathcal{U}\left(x_{k}\right)\right|^{-\frac{2}{n-2}}$. Since $x_{k}$ is a maximum point if $r_{k}=$ $\frac{1}{2} d_{\varepsilon}\left(x_{k}\right)$, we have that

$$
|\mathcal{U}(x)| \leq 2^{\frac{n-2}{2}}\left|\mathcal{U}\left(x_{k}\right)\right|
$$

for $x \in B_{r_{k}}\left(x_{k}\right)$. Since $w_{i, k}$ is a solution of 3.1$)$ which is regular and bounded, standard elliptic theory then implies that, after passing to a subsequence, $w_{i, k}$ converge in $C^{2}$ norm on compact subsets of $\mathbb{R}^{n}$ to a positive solution $u_{i, 0}$ to

$$
\Delta u_{i, 0}+\frac{n(n-2)}{4}\left|\mathcal{U}_{0}\right|^{\frac{4}{n-2}} u_{i, 0}=0
$$

which by a theorem due to Druet and Hebey [6] is the spherical standard solution.

Now let us prove that the bounds on $y_{0}$ and $\mu$ are valid. Indeed, from the previous argument we know that

$$
\left|\mathcal{W}_{\lambda}\right| \leq 2^{\frac{n-2}{2}} \text { in } B_{R^{\prime}}(0),
$$

where $R^{\prime}=d_{\varepsilon}\left(x_{0}\right) / 2 \eta \geq c_{1}^{\frac{2}{n-2}} / 2$. We can always assume that $c_{1} \geq 2^{n-2}$ and consequently $R^{\prime} \geq 2$. Then, it holds

$$
\left|\mathcal{U}_{\mu, y_{0}}(y)\right| \leq 2^{\frac{n-2}{2}}+\delta \leq 2^{\frac{n}{2}}
$$

for all $y \in B_{2}(0)$. On the other hand, we have

$$
\left|\mathcal{U}_{\mu, y_{0}}(0)\right| \geq 1-\delta \geq 1 / 2
$$

Note that the lower bound $2^{-\frac{2}{n-2}} \leq 2 \mu$ follows directly from 3.8 . In order to prove the upper bound suppose first that $y_{0} \in B_{2}(0)$ then, taking $y=y_{0}$ in (3.7), we 
get that $2 \mu \leq 2^{\frac{n}{n-2}}$. Otherwise, there would exist $y_{1} \in B_{2}(0)$ with $\left|y_{1}-y_{0}\right|^{2}=\left|y_{0}\right|^{2}-1$ and taking $y=y_{1}$ in 3.7 we have

$$
2^{-\frac{2}{n-2}} \mu \leq 1+\mu^{2}\left|y_{0}\right|^{2}-\mu^{2} \leq 2^{\frac{n}{n-2}} \mu-\mu^{2} .
$$

This implies that $\mu \leq 2^{\frac{n}{n-2}}$ and thus $\left|y_{0}\right| \leq 2^{n /(n-2)}$. This finishes the proof of the claim.

Observe now that, if we shift the center point $x_{0}$ to a new point $x_{1}$ which is within distance $2 c_{2} \eta$, the inequality $(3.6)$ can be replaced by

$$
\left\|u_{1,0} \Lambda_{i}-w_{\lambda}\right\|_{C^{2}\left(B_{R}(0)\right)}<\delta .
$$

Indeed, $\left|\mathcal{U}_{\mu, y_{0}}\right|$ has a nondegenerate maximum point at $y_{0}$. Then we conclude that for $k$ sufficiently large there exists a nondegenerate maximum point $y_{k}$ of $\left|\mathcal{W}_{k}\right|$ near $y_{0}$. Since $y_{0}$ and $\mu$ are bounded there will be a corresponding local maximum point $x_{1}$ of $\left|\mathcal{W}_{k}\right|$ satisfying $\left|x_{0}-x_{1}\right| \leq 2 c_{2} \eta<1 / 16$, increasing $c_{1}$ if necessary. If we redefine the functions $w_{i, k}$ replacing $x_{0}$ by $x_{1}$, the result follows.

In order to prove $(3.5)$, observe first that since $\mathcal{U}$ is a supersolution, $|\mathcal{U}|$ is locally bounded in $L^{(n+2) /(n-2)}\left(B^{n}\right)$, such function is superharmonic and thus it can be redefined on $\Gamma$ as to be upper semicontinuous, which implies that $\inf _{\partial B_{3 / 4}(0)}|\mathcal{U}|$ is bounded from above.

Let $R=e^{2}$ and $\delta \in\left(0, \frac{1}{2}\right]$ be fixed constants. If the left-hand side of inequality (3.5) is bounded by $c_{1}$ (given in the Claim 1), then the result follows directly. Suppose that this is not the case.

From now on we will work in cylindrical coordinates. If $\eta=\left|\mathcal{U}\left(x_{1}\right)\right|^{-\frac{2}{n-2}}$ then we have

$$
v_{i}(t, \theta)=|x|^{\frac{n-2}{2}} w_{i, \eta}(x),
$$

where $t=-\log |x|$ and $\theta=x /|x|$.

Since $x_{1}$ lies in the ball of radius $11 / 16$ about the origin, its distance to $\partial B_{3 / 4}(0)$ is at least $1 / 16$. Then the ball $B_{\eta^{-1} / 16}(0)$ is contained in the domain of $w_{i, \eta}$. Now define,

$$
\mathcal{V}_{1,0}(t)=|x|^{\frac{n-2}{2}} u_{1,0}(x) \Lambda=(\cosh t)^{\frac{2-n}{2}} \Lambda
$$

From $(3.9)$, we know that $\mathcal{V}$ is close in the $C^{2}$ norm on $[-2, \infty) \times \mathbb{S}^{n-1}$ to the function $\mathcal{V}_{1,0}$. Consequently $\partial_{t} v_{i}(-1, \theta)>0$ for all $\theta \in \mathbb{S}^{n-1}$. 
We will apply the Alexandrov technique to the coordinates $v_{i}$ on the region $\left[-\log \left(\frac{1}{16} \eta^{-1}\right), \infty\right) \times \mathbb{S}^{n-1}$, reflecting across the spheres $\{\alpha\} \times \mathbb{S}^{n-1}$ starting with $\alpha$ sufficiently large, and continuing as far as possible. Once $\partial_{t} v_{i}(-1, \theta)>0$, this procedure must end before $\alpha$ reachs -1 . Let

$$
\begin{aligned}
& v=v_{1}+v_{2}, \\
& v_{i, \alpha}(t, \theta)=v_{i}(2 \alpha-t, \theta)
\end{aligned}
$$

and

$$
v_{\alpha}(t, \theta)=v(2 \alpha-t, \theta)
$$

Then, the difference between the function $v_{i}$ and the reflection satisfies

$$
L\left(v_{i}-v_{i, \alpha}\right)+\frac{n(n-2)}{4}|\mathcal{V}|^{\frac{4}{n-2}} v_{i}-\frac{n(n-2)}{4}\left|\mathcal{V}_{\alpha}\right|^{\frac{4}{n-2}} v_{i, \alpha}=0
$$

Note that

$$
|\mathcal{V}|^{\frac{4}{n-2}} v-\left|\mathcal{V}_{\alpha}\right|^{\frac{4}{n-2}} v_{\alpha}=\sum_{j=1}^{2} b_{i, \alpha}\left(v_{i}-v_{i, \alpha}\right),
$$

where

$$
b_{i, \alpha}=v\left(v_{i}+v_{i, \alpha}\right) \frac{|\mathcal{V}|^{\frac{4}{n-2}}-\left|\mathcal{V}_{\alpha}\right|^{\frac{4}{n-2}}}{|\mathcal{V}|^{2}-\left|\mathcal{V}_{\alpha}\right|^{2}}+\left|\mathcal{V}_{\alpha}\right|^{\frac{4}{n-2}}>0
$$

By (3.10) we have

$$
L\left(v-v_{\alpha}\right)+\sum_{i=1}^{2} b_{i, \alpha}\left(v_{i}-v_{i, \alpha}\right)=0
$$

weakly. In order to conclude the proof, we will also need the following:

Claim 2: There exist $\alpha_{0}>-1$ and $\theta_{0} \in \mathbb{S}^{n-1}$ such that

$$
\left.v\left(-\log \left(\frac{1}{16} \eta^{-1}\right), \theta_{0}\right)=v_{\alpha_{0}}\left(-\log \left(\frac{1}{16} \eta^{-1}\right), \theta_{0}\right)\right) .
$$

Indeed, define

$$
\alpha_{0}=\inf \left\{\alpha_{1} ;\left(v-v_{\alpha}\right)(t, \theta) \geq 0 \text { in }\left[-\log \left(\frac{1}{16} \eta^{-1}\right), \alpha_{1}\right], \forall \alpha \geq \alpha_{1}\right\}
$$

By continuity, $\left(v-v_{\alpha_{0}}\right)(t, \theta) \geq 0$ in $\left[-\log \left(\frac{1}{16} \eta^{-1}\right), \alpha_{0}\right]$. If we suppose by contradiction that the claim is not true, then

$$
\left(v-v_{\alpha_{0}}\right)\left(-\log \left(\frac{1}{16} \eta^{-1}\right), \theta\right)>0
$$


for all $\theta \in \mathbb{S}^{n-1}$. Since the following inequality

$$
L\left(v-v_{\alpha_{0}}\right)-D\left(v-v_{\alpha_{0}}\right) \leq-\sum_{i=1}^{2} b_{i, \alpha_{0}}\left(v_{i}-v_{i, \alpha_{0}}\right) \leq 0,
$$

holds weakly, where $D$ is a positive function, this implies that $\left(v-v_{\alpha_{0}}\right)(t, \theta)>0$ for every $-\log \left((16 \eta)^{-1}\right)<t<\alpha_{0}$ and $\theta \in \mathbb{S}^{n-1}$, since on the boundary of $\left[-\log \left((16 \eta)^{-1}\right), \alpha_{0}\right]$ the difference is nonnegative.

From the definition of $\alpha_{0}$, there exist a sequence $\left\{\alpha_{j}\right\}$ such that $\alpha_{j}<\alpha_{0}$ and $\alpha_{j} \rightarrow \alpha_{0}$, and a sequence $\left\{\left(t_{j}, \alpha_{j}\right)\right\}$ of interior minimum points of $v-v_{\alpha_{j}}$ such that $\left(t_{j}, \theta_{j}\right) \rightarrow\left(t^{*}, \theta^{*}\right)$ with $\left(v-v_{\alpha_{j}}\right)\left(t_{j}, \theta_{j}\right)<0$. Taking the limit $j \rightarrow \infty$ we get $(v-$ $\left.v_{\alpha_{0}}\right)\left(t^{*}, \theta^{*}\right)=0$ and $\nabla\left(v-v_{\alpha_{0}}\right)\left(t^{*}, \theta^{*}\right)=0$. Therefore $t^{*}=\alpha_{0}$, which is a contradiction to Hopf's Lemma. This proves the Claim 2.

Hence we see that there exist $\alpha_{0}$ and $\theta_{0}$ such that

$$
\left.v\left(-\log \left(\frac{1}{16} \eta^{-1}\right), \theta_{0}\right)=v_{\alpha_{0}}\left(-\log \left(\frac{1}{16} \eta^{-1}\right), \theta_{0}\right)\right)
$$

which implies

$$
\inf \left\{v\left(-\log \left(\frac{1}{16} \eta^{-1}\right), \theta\right): \theta \in \mathbb{S}^{n-1}\right\} \leq \sup \left\{v(t, \theta): t>\log \left(\frac{1}{16} \eta^{-1}\right)-2 \alpha_{0}, \theta \in \mathbb{S}^{n-1}\right\}
$$

Note that since $\mathcal{U}$ is superharmonic, it holds

$$
\inf \left\{v\left(-\log \left(\frac{1}{16} \eta^{-1}\right), \theta\right): \theta \in \mathbb{S}^{n-1}\right\}=16^{\frac{2-n}{2}} \inf _{\partial B_{1 / 16}\left(x_{1}\right)} u \geq 16^{\frac{2-n}{2}} \inf _{\partial B_{3 / 4}(0)}|\mathcal{U}| .
$$

On the other hand, for $\eta$ small and fixed, we have $|\mathcal{V}|(t, \theta) \leq 2\left|\mathcal{V}_{1,0}\right|(t, \theta)$ for $t \geq 0$, and therefore

$$
\inf _{\partial B_{3 / 4}(0)}|\mathcal{U}| \leq c \sup _{\left[\log \left(\frac{1}{16} \eta^{-1}\right)-2 \alpha_{0}, \infty\right)}\left|\mathcal{V}_{1,0}\right| \leq c \eta^{\frac{n-2}{2}}\left|\mathcal{V}_{1,0}\right|=c\left|\mathcal{U}\left(x_{1}\right)\right|^{-1}
$$

Since $U_{\varepsilon}$ is bounded by a constant times $|\mathcal{U}|$, 2the inequality 3.5 is true, which finishes the proof of the result.

The following result follows directly as a consequence of Lemma 3.1 and Theorem 3.2 . 
Corollary 3.3 Assume that $\mathcal{U}$ is a nonnegative smooth solution of (3.1) defined in the punctured unit ball $B_{1}^{n}(0) \backslash\{0\}$. Either $\mathcal{U}$ extends as a smooth solution to the ball, or there exists positive constants $c>0$, such that

$$
|\mathcal{U}|(x) \leq c|x|^{\frac{2-n}{2}}
$$

for $0<|x|<1 / 2$.

Corollary 3.4 Suppose $\mathcal{U}$ is a positive smooth solution of (3.1) in $\Omega=B_{1}^{n}(0) \backslash\{0\}$. Then there exists a constant $c>0$ such that

$$
\max _{|x|=r} u_{i} \leq c \min _{|x|=r} u_{i}
$$

for every $0<r<\frac{1}{4}$. Moreover, $\left|\nabla u_{i}\right| \leq c|x|^{-1} u_{i}$ and $\left|\nabla^{2} u_{i}\right| \leq c|x|^{-2} u_{i}$.

Proof. Define $u_{i, r}(y)=r^{\frac{n-2}{2}} u_{i}(r y)$, for every $0<r<\frac{1}{4}$ and $|y|<r^{-1}$. Then the upper bound given by previous theorem implies that $u_{i, r}(y) \leq c|y|^{\frac{2-n}{2}}$, for $|y|<\frac{1}{2} r^{-1}$. In particular, if $\frac{1}{2} \leq|y| \leq \frac{3}{2}$, we have that $u_{i, r}(y) \leq 2^{\frac{n-2}{2}} c$. Moreover

$$
\Delta u_{i, r}(y)+\frac{n(n-2)}{4}\left|\mathcal{U}_{r}(y)\right|^{\frac{4}{n-2}} u_{i, r}(y)=0 .
$$

Hence the Harnack inequality for linear elliptic equations and standard elliptic theory imply there exists $c>0$, not depending on $r$, such that

$$
\max _{|x|=1} u_{i, r} \leq c \min _{|x|=1} u_{i, r}
$$

and $\left|\nabla u_{i, r}\right|+\left|\nabla^{2} u_{i, r}\right| \leq c u_{1, r}$ on the sphere of radius 1 . This finishes the proof of the corollary.

\subsection{Pohozaev invariant and lower bound}

In this section we will prove a lower bound for the solutions of (3.1) in the punctured ball and we will give some information about the Pohozaev invariant when the singularity is nonremovable.

We will begin by proving a Lemma that gives us a sufficient condition so that the origin can be a removable singularity.

Lemma 3.5 Let $\mathcal{U}$ be a positive solution of (3.1) defined in the punctured ball and denote $u(x)=u_{1}(x)+u_{2}(x)$. If

$$
\lim _{|x| \rightarrow 0}|x|^{\frac{n-2}{2}} u(x)=0,
$$

then $\mathcal{U}$ extends as a smooth solution to all of $B_{1}$. 
Proof. Observe first that Lemma 3.1 implies that $\mathcal{U}$ extends as a weak solution on the unit ball. By hypotesis (3.14) we know that $v(t, \theta)$ converges uniformly to zero as $t$ tends to $\infty$, where $v$ corresponds to $u$ by the change of variables of Fowler $(1.2)$. Since $v$ satisfies

$$
\partial_{t}^{2} v+\Delta_{\mathbb{S}^{n-1}} v-\frac{(n-2)^{2}}{4} v+\frac{n(n-2)}{4}|\mathcal{V}|^{\frac{4}{n-2}} v=0
$$

there exist $\beta>0$ and $t_{0}>0$ such that

$$
L v=\partial_{t}^{2} v+\Delta_{\mathbb{S}^{n-1}} v \geq \beta v
$$

for $t \geq t_{0}$. Consider now the function

$$
w=c e^{-\sqrt{\beta t}}+\varepsilon e^{\sqrt{\beta t}}
$$

where $c$ is chosen such that $c e^{-\sqrt{\beta t_{0}}}>v\left(t_{0}, \theta\right)$ for all $\theta \in \mathbb{S}^{n-1}$. Note that $w$ satisfies $L w=\beta w$. Since $w$ is growing at an exponential rate and $v$ is converging to zero, we can choose sufficiently large $T$ such that $v(T, \theta)<w(T, \theta)$ for all $\theta$ in the unit sphere.

Aplying the maximum principle we get for all $t_{0} \leq t \leq T$ and for all $\theta$ that

$$
v(t, \theta) \leq c e^{-\sqrt{\beta t}}+\varepsilon e^{\sqrt{\beta t}} .
$$

Since $T$ was choosen arbitrarily large, this inequality holds for all $t \geq t_{0}$. Making $\varepsilon$ converge to zero, we conclude that for all $t \geq t_{0}$

$$
e^{\sqrt{\beta t}} v(t, \theta) \leq c
$$

which implies $u(x) \leq c|x|^{q}$ for $q=(2-n) / 2+\sqrt{\beta}$. This estimate shows that $u \in$ $L_{l o c}^{p}\left(B_{1}(0)\right)$ for arbitrarily large $p$ and then by elliptic theory the function $u$ extends smoothly across the origin. Consequently, each coordinate function $u_{i}$ is smooth.

Similarly to what we did in the previous chapter we can define for $r>0$ the Pohozaev integral as

$$
P(r, \mathcal{U})=\int_{\partial B_{r}}\left(\frac{n-2}{2}\left\langle\mathcal{U}, \frac{\partial \mathcal{U}}{\partial \nu}\right\rangle-\frac{r}{2}|\nabla \mathcal{U}|^{2}+r\left|\frac{\partial \mathcal{U}}{\partial \nu}\right|^{2}+r \frac{(n-2)^{2}}{8}|\mathcal{U}|^{\frac{2 n}{n-2}}\right) d \sigma
$$

where $\nu$ is the unit outer normal of $\partial B_{r}$. Multiplying each equation of (3.1) by $x \cdot \nabla u_{i}$ and integrating over $B_{r} \backslash B_{s}$ we verify for all $r, s>0$ that

$$
P(r, \mathcal{U})=P(s, \mathcal{U})
$$

and hence we can denote it by $P(\mathcal{U})$ and call it the Pohozaev invariant. 
Theorem 3.6 Assume that $\mathcal{U}$ is a nonnegative smooth solution of (3.1) defined in the punctured unit ball $B_{1}^{n}(0) \backslash\{0\}$. Either $\mathcal{U}$ extends as a smooth solution to the ball, or there exists a positive constant $c$ such that

$$
|\mathcal{U}|(x) \geq c|x|^{\frac{2-n}{2}}
$$

In the latter case the radial Pohozaev invariant of $\mathcal{U}$ is negative.

Proof. Again it will be more convinient work in the cylindrical background. If $v=$ $v_{1}+v_{2}$, define

$$
\bar{v}(t)=\int_{\mathbb{S}^{n-1}} v(t, \theta) d \theta .
$$

As a consequence of the spherical Harnack inequality given by Corollary 3.4, we know that $v / \bar{v}$ is bounded from above. Suppose that the solution $\mathcal{U}$ can not be extended smoothly across the orign. By Lemma 3.5, this imply that either the claimed lower bound holds, or there exists a sequence $t_{k}$ of critical points of $v$ such that $\lim _{k \rightarrow \infty} \bar{v}\left(t_{k}\right)=$ 0 .

On one hand, in the cylindrical setting the Pohozaev integral $P\left(t_{k}, \mathcal{V}\right)=P\left(e^{-t_{k}}, \mathcal{U}\right)$ is given by

$$
P\left(t_{k}, \mathcal{V}\right):=\int_{t_{k} \times \mathbb{S}^{n-1}}\left(\frac{1}{2}\left|\partial_{t} \mathcal{V}\right|^{2}-\frac{1}{2}\left|\nabla_{\theta} \mathcal{V}\right|^{2}-\frac{(n-2)^{2}}{8}|\mathcal{V}|^{2}+\frac{(n-2)^{2}}{8}|\mathcal{V}|^{\frac{2 n}{n-2}}\right) d \sigma
$$

Consequently using the fact that the Pohozaev integral is independent of $k$, the Harnack inequality, standard elliptic estimates as well as the fact that $v_{i}$ is a solution of 3.2 imply that the invariant must vanish.

On the other hand, consider the sequence of functions

$$
w_{i, k}(t, \theta)=\frac{v_{i}\left(t+t_{k}, \theta\right)}{\bar{v}\left(t_{k}\right)}
$$

Using elliptic estimates, we can extract a subsequence of $w_{i, k}$ which converges in $C^{2}$ topology on compact subsets to a positive solution of $L_{c y l} w_{i}=0$. In euclidian coordinates the associated function $h_{i}(x)$ defined on $\mathbb{R}^{n} \backslash\{0\}$ is then a positive harmonic function, and can therefore be written as $h_{i}(x)=a_{i}|x|^{2-n}+b_{i}$, for some $a_{i}, b_{i} \geq 0$. That is,

$$
w_{i}(t, \theta)=a_{i} e^{\frac{n-2}{2} t}+b_{i} e^{-\frac{n-2}{2} t} .
$$


We also know from the fact that the sequence $\left\{t_{k}\right\}$ was choosen as critical points for $\bar{v}$ that $a_{1}+a_{2}=b_{1}+b_{2}$ is positive. Then, by a direct computation

$$
\begin{array}{r}
\lim _{k \rightarrow \infty} \int_{\mathbb{S}^{n-1}}\left[\sum_{i}\left(\frac{1}{2}\left(\partial_{t} w_{i, k}\right)^{2}-\frac{1}{2}\left|\nabla_{\theta} w_{i, k}\right|^{2}-\frac{(n-2)^{2}}{8} w_{i, k}^{2}\right)+\bar{v}\left(t_{k}\right)^{\frac{4}{n-2}}\left|\mathcal{W}_{k}\right|^{\frac{2 n}{n-2}}\right] d \theta \\
=\int_{\mathbb{S}^{n-1}} \sum_{i}\left(\frac{1}{2}\left(\partial_{t} w_{i}\right)^{2}-\frac{(n-2)^{2}}{8} w_{i}^{2}\right) d \theta=-\omega_{n} \frac{(n-2)^{2}}{2}\langle a, b\rangle .
\end{array}
$$

We claim that $\langle a, b\rangle$ is positive. Indeed, if we consider the function

$$
z_{i, k}(x)=|x|^{\frac{2-n}{2}} w_{i, k}(-\log |x|, x /|x|)
$$

one verifies that $\mathcal{Z}_{k}=\left(z_{1, k}, z_{2, k}\right)$ satisfies the following system

$$
\Delta z_{i, k}+\frac{n(n-2)}{4}\left|\mathcal{Z}_{k}\right|^{\frac{4}{n-2}} z_{i, k}=0 \quad \text { in } \quad B_{r_{k}-1}(0) \backslash\{0\}
$$

where $r_{k}=e^{-t_{k}}$.

Now, multiplying line $i$ of system $(3.17)$ by $z_{j, k}$ and integrating over $B_{1}(0) \backslash B_{\varepsilon}(0)$, we obtain that

$$
\int_{B_{1} \backslash B_{\varepsilon}} z_{j, k} \Delta z_{i, k} d x=\frac{n(n-2)}{4} \int_{B_{1} \backslash B_{\varepsilon}}\left|\mathcal{Z}_{k}\right|^{\frac{4}{n-2}} z_{i, k} z_{j, k} d x
$$

Reversing $i$ and $j$ and subtracting one equation from the other give us

$$
\int_{B_{1} \backslash B_{\varepsilon}}\left(z_{j, k} \Delta z_{i, k}-z_{i, k} \Delta z_{j, k}\right) d x=0,
$$

which, integrating by parts, guarantees that

$$
\int_{\partial B_{1}}\left(z_{j, k} \partial_{r} z_{i, k}-z_{i, k} \partial_{r} z_{j, k}\right) d x=\int_{\partial B_{\varepsilon}}\left(z_{j, k} \partial_{r} z_{i, k}-z_{i, k} \partial_{r} z_{j, k}\right) d x .
$$

In order to analyse the last integral on the right-hand side, let

$$
\varphi_{i, k}^{\varepsilon}(y)=\varepsilon^{\frac{n-2}{2}} z_{i, k}(\varepsilon y) .
$$

It holds

$$
\int_{\partial B_{\varepsilon}}\left(z_{j, k} \partial_{r} z_{i, k}-z_{i, k} \partial_{r} z_{j, k}\right) d x=\int_{\partial B_{1}}\left(\varphi_{j, k}^{\varepsilon} \partial_{r} \varphi_{i, k}^{\varepsilon}-\varphi_{i, k}^{\varepsilon} \partial_{r} \varphi_{i, k}^{\varepsilon}\right) d x
$$

Since

$$
\Delta \varphi_{i, k}^{\varepsilon}+\frac{n(n-2)}{4}\left|\varphi_{k}^{\varepsilon}\right|^{\frac{4}{n-2}} \varphi_{i, k}^{\varepsilon}=0
$$


in $B_{\left(\varepsilon r_{k}\right)^{-1}}(0) \backslash\{0\}$, by Corollary 3.3 we get

$$
\left|\varphi_{k}^{\varepsilon}(y)\right| \leq C|y|^{\frac{2-n}{2}} .
$$

By standard elliptic estimates, after passing to a subsequence, $\varphi_{i, k}^{\varepsilon}$ converges in $C^{2}$ topollogy locally in compact subsets of $\mathbb{R}^{n} \backslash\{0\}$, to a positive solution

$$
\Delta u_{i}+\frac{n(n-2)}{4}|\mathcal{U}|^{\frac{4}{n-2}} u_{i}=0
$$

which using Theorem 2.10 as well as [7, Proposition 1.1] is of the form $u_{0} \Lambda$, where $\Lambda=\left(\Lambda_{1}, \Lambda_{2}\right)$ is a vector in the unit sphere with nonnegative coordinates. Hence

$$
\lim _{\varepsilon \rightarrow 0} \int_{\partial B_{1}}\left(\varphi_{j, k}^{\varepsilon} \partial_{r} \varphi_{i, k}^{\varepsilon}-\varphi_{i, k}^{\varepsilon} \partial_{r} \varphi_{i, k}^{\varepsilon}\right) d \sigma_{g}=\int_{\partial B_{1}}\left(u_{0} \partial_{r} u_{0} \Lambda_{i} \Lambda_{j}-u_{0} \partial_{r} u_{0} \Lambda_{j} \Lambda_{i}\right) d \sigma_{g}=0 .
$$

Making $\varepsilon \rightarrow 0$ in 3.18 , we conclude that

$$
\int_{\partial B_{1}}\left(z_{j, k} \partial_{r} z_{i, k}-z_{i, k} \partial_{r} z_{j, k}\right) d x=0 .
$$

Finally, we conclude that

$$
\int_{\partial B_{1}}\left(h_{j} \partial_{r} h_{i}-h_{i} \partial_{r} h_{j}\right) d \sigma_{g}=0
$$

and consequently

$$
a_{1} b_{2}=a_{2} b_{1}
$$

which finishes the proof of our claim.

Note now that this is a contradiction, for this is also a limit of rescalings of the Pohozaev invariant for $\mathcal{V}$, and hence must be zero. Indeed

$$
\begin{aligned}
& 0=\bar{v}\left(t_{k}\right)^{-2} P(\mathcal{V})= \\
& \int_{\mathbb{S}^{n-1}}\left[\sum_{i}\left(\frac{1}{2}\left(\partial_{t} w_{i, k}\right)^{2}-\frac{1}{2}\left|\nabla_{\theta} w_{i, k}\right|^{2}-\frac{(n-2)^{2}}{8} w_{i, k}^{2}\right)+\bar{v}\left(t_{k}\right)^{\frac{4}{n-2}}\left|\mathcal{W}_{k}\right|^{\frac{2 n}{n-2}}\right] d \theta \neq 0,
\end{aligned}
$$

for sufficiently large $k$. This estabilishes the lower bound.

In order to show that the Pohozaev invariant is negative, by the upper and lower bounds, we can choose a sequence $\tilde{t}_{k}$ tending to $\infty$ so that the corresponding translated solutions $t \rightarrow \mathcal{V}\left(t+t_{k}, \theta\right)$ converge in $C^{2}$ norm on compact subsets of $\mathbb{R} \times \mathbb{S}^{n-1}$ to a Fowler-type solution $\mathcal{V}_{0}$ satisfying the same bounds and defined on all of $\mathbb{R} \times \mathbb{S}^{n-1}$. Therefore

$$
P(\mathcal{V})=P\left(\mathcal{V}_{0}\right)<0
$$

which finishes the proof. 


\subsection{Simple convergence to a radial solution}

Our main goal in this section is to prove that a singular solution to the system (3.1) is asymptotic to a radial Fowler-type solution near the nonremovable isolated singularity.

Theorem 3.7 Suppose that $\mathcal{U}$ is a solution of the system (3.1) in the punctured ball $B_{1}^{n}(0) \backslash\{0\}$. If there exist positive constants $c_{1}$ and $c_{2}$ such that

$$
c_{1}|x|^{\frac{2-n}{2}} \leq|\mathcal{U}|(x) \leq c_{2}|x|^{\frac{2-n}{2}}
$$

then there exist a Fowler-type solution $\mathcal{U}_{0}=u_{0} \Lambda$ of (6), where $u_{0}$ is a Fowler solution such that

$$
\mathcal{U}(x)=\left(1+O\left(|x|^{\alpha}\right)\right) \mathcal{U}_{0}(x)
$$

as $x \rightarrow 0$, for some $\alpha>0$.

Proof. First we observe that 3.20 implies that the origin is a nonremovable singularity. Thus, we get that the Pohozaev invariant is negative.

Consider $v_{i}$ obtained from $u_{i}$ by the change of variables from Fowler and let $\left\{\tau_{k}\right\}$ be a sequence of real numbers such that $\tau_{k} \rightarrow \infty$. Consider the translated sequence $v_{i, k}(t, \theta)=v_{i}\left(t+\tau_{k}, \theta\right)$ defined in $\left(-\tau_{k}, \infty\right) \times \mathbb{S}^{n-1}$. By 3.20$)$, we get that

$$
c_{1} \leq\left|\mathcal{V}_{k}(t, \theta)\right| \leq c_{2}
$$

where $\mathcal{V}_{k}=\left(v_{1, k}, v_{2, k}\right)$. Consequently, by standard elliptic estimates, we get the uniform boundedness of any derivative for $t>0$ and moreover there exists a subsequence, also denoted by $v_{i, k}$, which converges in the $C_{l o c}^{2}$ topology, to a positive solution of

$$
L_{c y l} v_{i, 0}+\frac{n(n-2)}{4}\left|\mathcal{V}_{0}\right|^{\frac{4}{n-2}} v_{i, 0}=0
$$

defined in the whole cylinder. By the characterization result given by Theorem 2.10 , such limit is a Fowler-type solution and we know that there exists a Fowler solution $v_{\varepsilon}$ and a vector in the unit sphere with positive coordinates $\Lambda$ such that $\mathcal{V}_{\varepsilon}(t)=\Lambda v_{\varepsilon}(t)$. Hence $\mathcal{V}_{\varepsilon}$ does not depend on $\theta$, and we necessarily have that any angular derivative $\partial_{\theta} v_{i, k}$ converges uniformly to zero.

Besides, we claim that

$$
\begin{aligned}
v_{i, k}(t, \theta) & =\bar{v}_{i, k}(t)(1+o(1)) \\
\nabla v_{i, k}(t, \theta) & =-\bar{v}_{i, k}^{\prime}(t)(1+o(1)),
\end{aligned}
$$


as $t \rightarrow \infty$. In fact, suppose that the first equality above is false. Then there exist $\bar{\varepsilon}>0$ and sequences $\tau_{k} \rightarrow \infty, \theta_{k} \rightarrow \theta \in \mathbb{S}^{n-1}$ such that

$$
\left|\frac{v_{i, k}\left(\tau_{k}, \theta_{k}\right)}{\bar{v}_{i, k}\left(\tau_{k}\right)}-1\right| \geq \bar{\varepsilon}
$$

for some $i \geq 1$. This is a contradiction because, after passing to a subsequence, $\mathcal{V}_{k}$ converges to a rotationally symmetric Fowler-type solution $\mathcal{V}_{0}$. The second inequality follows from similar arguments.

Note now that

$$
P\left(\mathcal{V}_{\varepsilon}\right):=P\left(0, \mathcal{V}_{\varepsilon}\right)=\lim _{k \rightarrow \infty} P\left(0, \mathcal{V}_{k}\right)=\lim _{k \rightarrow \infty} P\left(\tau_{k}, \mathcal{V}\right)=P(\mathcal{V})
$$

So we can conclude that the necksize $\varepsilon$ of the limit function is independent of the sequence of numbers $\tau_{k}$. Therefore, for each sequence $\tau_{k} \rightarrow \infty$ the correspondent sequence $\mathcal{V}_{k}$ converges to a function $\mathcal{V}_{\varepsilon, T}(t)=\Lambda v_{\varepsilon}(t+T)$, with $\Lambda \in \mathbb{S}_{+}^{1}$, for some $T \in \mathbb{R}$ which depends on the sequence $\tau_{k}$.

We will show that there exists $T_{0} \in \mathbb{R}$ such that $\mathcal{V}_{k}$ converges to $\mathcal{V}_{\varepsilon, T_{0}}$ for any sequence $\tau_{k} \rightarrow \infty$. The ideia is to use a delicate rescaling argument due originally to Leon Simon. In order to do that we will prove several claims using the Jacobi fields studied in section 2.5 as a tool.

Let $T_{\varepsilon}$ be the period of $\mathcal{V}_{\varepsilon}$ and $A_{\tau}=\sup _{t>0}\left|\partial_{\theta} \mathcal{V}_{\tau}\right|$, where $\mathcal{V}_{\tau}(t, \theta)=\mathcal{V}(t+\tau, \theta)$. Note that $A_{\tau}<\infty$, since $\left|\partial_{\theta} \mathcal{V}_{\tau}\right|$ converges uniformly to zero as $t \rightarrow \infty$.

Claim 1: For every $c>0$, there exists a positive integer $N$ such that, for any $\tau>0$, either

(i) $A_{\tau} \leq c e^{-2 \tau}$ or

(ii) $A_{\tau}$ is attained at some point in $I_{N} \times \mathbb{S}^{n-1}$, where $I_{N}=\left[0, N T_{\varepsilon}\right]$.

Suppose the Claim is not true. Then there exist a constant $c>0$ and sequences $\tau_{k}, s_{k} \rightarrow \infty, \theta_{k} \in \mathbb{S}^{n-1}$ such that $\left|\partial_{\theta} \mathcal{V}_{\tau}\right|\left(s_{k}, \theta_{k}\right)=A_{\tau_{k}}$ and $A_{\tau_{k}}>c e^{-2 \tau_{k}}$ as $k \rightarrow \infty$. Then we can translate back further $s_{k}$ and define $\tilde{v}_{i, k}(t, \theta)=v_{i, k}\left(t+s_{k}, \theta\right)$. Define $\varphi_{i, k}=A_{\tau_{k}}^{-1} \partial_{\theta} \tilde{v}_{i, k}$ and note that $\left|\varphi_{\boldsymbol{k}}\right| \leq 1$, where $\varphi_{\boldsymbol{k}}=\left(\varphi_{1, k}, \varphi_{2, k}\right)$. Now, we have

$$
L_{c y l}\left(\tilde{v}_{i, k}\right)+\frac{n(n-2)}{4}\left|\tilde{\mathcal{V}}_{k}\right|^{\frac{4}{n-2}} \tilde{v}_{i, k}=0
$$


where the quantities with tilde are the originals replacing $t$ by $t+\tau_{k}+s_{k}$. Taking the derivative with respect to $\theta$ and multipling by $A_{\tau_{k}}^{-1}$, we get

$$
L_{c y l}\left(\varphi_{i, k}\right)+\frac{n(n-2)}{4}\left|\tilde{\mathcal{V}}_{k}\right|^{\frac{4}{n-2}} \varphi_{i, k}+n\left|\tilde{\mathcal{V}}_{k}\right|^{\frac{4}{n-2}-2} \tilde{v}_{i k}\left\langle\tilde{\mathcal{V}}_{k}, \varphi_{\boldsymbol{k}}\right\rangle=0
$$

Now we can use elliptic theory to extract a subsequence $\varphi_{i, k}$ which converges in compact subsets to a nontrivial and bounded Jacobi field $\varphi=\left(\varphi_{1}, \varphi_{2}\right)$ which satisfies the following system

$$
L_{c y l}\left(\varphi_{i}\right)+\frac{n(n-2)}{4} v_{\varepsilon}^{\frac{4}{n-2}} \varphi_{i}+n \Lambda_{i}\langle\Lambda, \varphi\rangle v_{\varepsilon}^{\frac{4}{n-2}}=0 .
$$

Since each coordinate function of the limit $\varphi_{i}$ has no zero eigencomponent relative to $\Delta_{\theta}$, we get a contradiction because a Jacobi field with such property is necessarily unbounded. This proves the Claim 1 .

Now suppose we have a sequence $v_{i, k}(t, \theta)$ converging to $\Lambda_{i} v_{\varepsilon}(t+T)$ as $k \rightarrow \infty$. Define

$$
w_{i, k}(t, \theta)=v_{i, k}(t, \theta)-\Lambda_{i} v_{\varepsilon}(t+T)
$$

and set

$$
\eta_{k}=b \max _{I_{N}}\left|\left(w_{1, k}, w_{2, k}\right)\right| \quad \text { and } \quad \varphi_{i, k}=\eta_{k}^{-1} w_{i, k},
$$

where $b>0$ is a fixed number to be chosen later and so that $\left|\left(\varphi_{1, k}, \varphi_{2, k}\right)\right| \leq b^{-1}$ on $I_{N}$. Then

$$
L_{c y l}\left(w_{i, k}\right)+\frac{n(n-2)}{4}\left(\left|\mathcal{V}_{k}\right|^{\frac{4}{n-2}} v_{i, k}-\Lambda_{i} v_{\varepsilon}^{\frac{n+2}{n-2}}\right)=0
$$

and

$$
\left|\mathcal{V}_{k}\right|^{\frac{4}{n-2}} v_{i, k}-\Lambda_{i} v_{\varepsilon}^{\frac{n+2}{n-2}}=\left|\mathcal{V}_{k}\right|^{\frac{4}{n-2}} w_{i, k}+\Lambda_{i} v_{\varepsilon} \frac{\left|\mathcal{V}_{k}\right|^{\frac{4}{n-2}}-v_{\varepsilon}^{\frac{4}{n-2}}}{\left|\mathcal{V}_{k}\right|^{2}-v_{\varepsilon}^{2}} \sum_{j} w_{j, k}\left(v_{j, k}+\Lambda_{i} v_{\varepsilon}\right)
$$

Multipling (3.23) by $\eta_{k}^{-1}$ and taking the limit $k \rightarrow \infty$ we get

$$
L_{c y l}\left(\varphi_{i}\right)+\frac{n(n-2)}{4} v_{0}^{\frac{4}{n-2}} \varphi_{i}+n \Lambda_{i}\langle\Lambda, \varphi\rangle v_{\varepsilon}^{\frac{4}{n-2}}=0,
$$

on the whole cylinder, where $\boldsymbol{\varphi}=\left(\varphi_{1}, \varphi_{2}\right)$ is a Jacobi field.

Claim 2: The Jacobi field $\varphi$ is bounded for $t \geq 0$.

To prove this claim we will use the analysis done in section 2.5. By the spectral decomposition for the laplacian in the sphere, we know that it is possible to write the Jacobi field as

$$
\boldsymbol{\varphi}=a_{1} \phi_{\varepsilon, 0}^{1}+a_{2} \phi_{\varepsilon, 0}^{2}+a_{3} \phi_{\varepsilon, 0}^{3}+a_{4} \phi_{\varepsilon, 0}^{4}+\tilde{\boldsymbol{\varphi}}
$$


where $\phi_{\varepsilon, 0}^{i}$ are the linearly independent Jacobi fields corresponding to the eigencomponent independent of $\theta$, and $\tilde{\varphi}$ denotes the projection onto the orthogonal complement. We also know that the functions $\phi_{\varepsilon, 0}^{1}$ and $\phi_{\varepsilon, 0}^{3}$ are bounded and $\phi_{\varepsilon, 0}^{2}$ and $\phi_{\varepsilon, 0}^{4}$ are linearly growing.

Let us show that $\tilde{\boldsymbol{\varphi}}$ is bounded by proving that each $\partial_{\theta} \tilde{\varphi}_{i}=\partial_{\theta} \varphi_{i}$ is bounded for $t \geq 0$. In fact, the function $\partial_{\theta} \varphi_{i}$ is the limit of $\eta_{k}^{-1} \partial_{\theta} v_{i, k}$, and we can suppose that $\partial_{\theta} \varphi_{i}$ is nontrivial, otherwise the result is immediate.

If the first item of Claim 1 happens then

$$
\sup _{t \geq 0}\left(\eta_{k}^{-1}\left|\partial_{\theta} v_{i, k}\right|\right) \leq c \eta_{k}^{-1} e^{-2 \tau_{k}} \leq C .
$$

While if the second item of Claim 1 is true then

$$
\sup _{t \geq 0}\left(\eta_{k}^{-1}\left|\partial_{\theta} v_{i, k}\right|\right) \leq \sup _{t \geq 0}\left(\eta_{k}^{-1}\left|\partial_{\theta} \mathcal{V}_{k}\right|\right)=\sup _{t \in I_{N}}\left(\eta_{k}^{-1}\left|\partial_{\theta} \mathcal{V}_{k}\right|\right) \leq C
$$

since the sequence $\eta_{k}^{-1}\left|\partial_{\theta} \mathcal{V}_{k}\right|$ converges in the $C_{l o c}^{2}$ toplogy. Therefore each $\tilde{\varphi}_{i}$ is bounded for $t \geq 0$, hence exponentially decaying.

To finish the proof of the Claim 2 we need to show that $a_{2}=a_{4}=0$. To see this note that the convergence $\varphi_{i, k}=\eta_{k}^{-1} w_{i, k} \rightarrow \varphi_{i}$ implies that

$$
\begin{aligned}
\mathcal{V}_{k} & =\Lambda v_{\varepsilon, T}+\eta_{k} \boldsymbol{\varphi}+o\left(\eta_{k}\right) \\
& =\Lambda v_{\varepsilon, T}+\eta_{k}\left(a_{1} \phi_{\varepsilon, 0}^{1}+a_{2} \phi_{\varepsilon, 0}^{2}+a_{3} \phi_{\varepsilon, 0}^{3}+a_{4} \phi_{\varepsilon, 0}^{4}+\tilde{\boldsymbol{\varphi}}\right)+o\left(\eta_{k}\right),
\end{aligned}
$$

where $v_{\varepsilon, T}(t)=v_{\varepsilon}(t+T)$. On the other hand we have that

$$
P\left(0, \mathcal{V}_{k}\right)=P\left(\tau_{k}, \mathcal{V}\right)=P(\mathcal{V})+O\left(e^{-2 \tau_{k}}\right)=P\left(v_{\varepsilon, T}\right)+O\left(e^{-2 \tau_{k}}\right)
$$

Since $\lim _{k \rightarrow \infty}\left(\eta_{k}^{-1} e^{-2 \tau_{k}}\right)=0$, we would have a contradiction in case $a_{2}$ or $a_{4}$ is not zero because the two sides of the last equality would differ for sufficiently large $k$. Thus each $\varphi$ is bounded for $t \geq 0$.

Now we show that there exists some $T$ so that the difference between $\mathcal{V}$ and $\mathcal{V}_{\varepsilon, T}=\Lambda v_{\varepsilon, T}$ goes to zero as $t \rightarrow \infty$. Since we do not know the correct translation parameter, define $\mathcal{V}_{\tau}(t, \theta)=\mathcal{V}(t+\tau, \theta)$ and $\mathcal{W}_{\tau}(t, \theta)=\mathcal{V}_{\tau}(t, \theta)-\Lambda v_{\varepsilon}(t)$. Let $C_{1}>0$ be a fixed constant and consider the interval $I_{N}$ as in the Claim 1. Set also $\eta(\tau)=b \max _{I_{N}}\left|\mathcal{W}_{\tau}\right|$, where $b>0$ is again a positive constant to be chosen later. We observe that $\eta(\tau) \rightarrow 0$ as $\tau \rightarrow \infty$. Let us prove the following claim. 
Claim 3: If $N, b$ and $\tau$ are sufficiently large and $\eta$ is sufficiently small, then there exists $s$ with $|s| \leq C_{1} \eta(\tau)$ so that $\eta\left(\tau+N T_{\varepsilon}+s\right) \leq \frac{1}{2} \eta(\tau)$.

Suppose the claim is not true. Then there exists some sequence $\tau_{k} \rightarrow \infty$ such that $\eta\left(\tau_{k}\right) \rightarrow 0$ and for any $s$ satisfying $|s| \leq C_{1} \eta\left(\tau_{k}\right)$ we have that $\eta\left(\tau_{k}+N T_{\varepsilon}+s\right)>\frac{1}{2} \eta\left(\tau_{k}\right)$. Define $\varphi_{i . k}=\eta\left(\tau_{k}\right)^{-1} w_{i, \tau_{k}}$, similarly to the previous claim. We can suppose that $\varphi_{i, k}$ converges in $C^{\infty}$ on compact sets to a Jacobi field, which by Claim 2 is bounded for $t \geq 0$. So we can write

$$
\boldsymbol{\varphi}=a_{1} \phi_{\varepsilon, 0}^{1}+a_{3} \phi_{\varepsilon, 0}^{3}+\tilde{\varphi}
$$

where $\tilde{\boldsymbol{\varphi}}$ has exponential decay. Note that $|\boldsymbol{\varphi}| \leq b^{-1}$ on $I_{N}$, which implies that $a_{1}$ and $a_{3}$ are uniformily bounded, independently of the sequence $\tau_{k}$. Moreover, since $\phi_{\varepsilon, 0}^{1}=v_{\varepsilon}^{\prime} \Lambda$ and $\phi_{\varepsilon, 0}^{3}=v_{\varepsilon} \bar{\Lambda}$ we have

$$
\left|a_{3} v_{\varepsilon}\right| \leq|\langle\boldsymbol{\varphi}, \bar{\Lambda}\rangle|+|\langle\tilde{\boldsymbol{\varphi}}, \bar{\Lambda}\rangle| \leq b^{-1}+|\tilde{\boldsymbol{\varphi}}|
$$

on $I_{N}$. We know that $v_{\varepsilon} \geq \varepsilon$ and $\tilde{\varphi}$ decreases exponentially with a fixed rate, and so we can choose $b$ and $N$ sufficiently large such that $\left|a_{3}\right|$ is sufficiently small.

Set $s_{k}=-\eta\left(\tau_{k}\right) a_{1}$ whose absolute value is less than $C_{1} \eta\left(\tau_{k}\right)$ if we choose $C_{1}$ sufficiently large. Hence for $t \in\left[0,2 N T_{\varepsilon}\right]$ we have

$$
\begin{aligned}
\mathcal{W}_{\tau_{k}+s_{k}}(t, \theta)= & \mathcal{V}\left(t+\tau_{k}-\eta\left(\tau_{k}\right) a_{1}, \theta\right)-\Lambda v_{\varepsilon}(t) \\
= & \mathcal{V}_{\tau_{k}}\left(t-\eta\left(\tau_{k}\right) a_{1}, \theta\right)-\Lambda v_{\varepsilon}\left(t-\eta\left(\tau_{k}\right) a_{1}\right) \\
& -\eta\left(\tau_{k}\right) a_{1} \Lambda \frac{v_{\varepsilon}\left(t-\eta\left(\tau_{k}\right) a_{1}\right)-v_{\varepsilon}(t)}{-\eta\left(\tau_{k}\right) a_{1}} \\
= & \eta\left(\tau_{k}\right) \boldsymbol{\varphi}_{k}\left(t-\eta\left(\tau_{k}\right) a_{1}, \theta\right)-\eta\left(\tau_{k}\right) a_{1} \phi_{\varepsilon, 0}^{1}+o\left(\eta\left(\tau_{k}\right)\right) \\
= & \mathcal{W}_{\tau_{k}}(t, \theta)-\eta\left(\tau_{k}\right) a_{1} \phi_{\varepsilon, 0}^{1}+o\left(\eta\left(\tau_{k}\right)\right),
\end{aligned}
$$

where $\boldsymbol{\varphi}_{k}=\left(\varphi_{1, k}, \varphi_{2, k}\right)$. Here we used the equality $\mathcal{W}_{\tau_{k}}=\eta\left(\tau_{k}\right) \boldsymbol{\varphi}_{k}+o\left(\eta\left(\tau_{k}\right)\right)$ and the fact that $\boldsymbol{\varphi}_{k}\left(t-\eta\left(\tau_{k}\right) a_{1}, \theta\right)-\boldsymbol{\varphi}_{k}(t, \theta)$ goes to zero as $\tau_{k} \rightarrow \infty$.

Consequently, by (3.24), for $t \in\left[0,2 N T_{\varepsilon}\right]$ we get that

$$
\mathcal{W}_{\tau_{k}+s_{k}}=\eta\left(\tau_{k}\right) \tilde{\boldsymbol{\varphi}}+\eta\left(\tau_{k}\right) a_{3} \phi_{\varepsilon, 0}^{3}+o\left(\eta\left(\tau_{k}\right)\right)
$$

which implies

$$
\max _{I_{N}}\left|\mathcal{W}_{\tau_{k}+s_{k}+N T_{\varepsilon}}\right|=\max _{\left[N T_{\varepsilon}, 2 N T_{\varepsilon}\right]}\left|\mathcal{W}_{\tau_{k}+s_{k}}\right| \leq \eta\left(\tau_{k}\right) \max _{\left[N T_{\varepsilon}, 2 N T_{\varepsilon}\right]}\left(|\tilde{\boldsymbol{\varphi}}|+\left|a_{3} v_{\varepsilon}\right|\right)+o\left(\eta\left(\tau_{k}\right)\right)
$$


Since $\tilde{\varphi}$ decreases exponentially with a fixed rate, by 3.25 we can choose $N$ and $b>0$ suficiently large in a way that the last equality implies that

$$
\max _{I_{N}}\left|\mathcal{W}_{\tau_{k}+s_{k}+N T_{\varepsilon}}\right| \leq \frac{1}{4} \eta\left(\tau_{k}\right)
$$

which on its turn gives $\eta\left(\tau+N T_{\varepsilon}+s\right) \leq \frac{1}{2} \eta(\tau)$, a contradiction. This ends the proof of the Claim 3.

Once the claim above is proved, using an iterative argument, we are ready to prove that there exists $\sigma$ such that $w_{i, \sigma} \rightarrow 0$ as $t \rightarrow \infty$ for each coordinate. First choose $\tau_{0}$ and $N$ sufficiently large satisfying the Claim 3 and such that $C_{1} \eta\left(\tau_{0}\right) \leq \frac{1}{2} N T_{\varepsilon}$. Let $s_{0}=-\eta\left(\tau_{0}\right) a_{1}$ be chosen as above. Thus we have $\left|s_{0}\right| \leq C_{1} \eta\left(\tau_{0}\right) \leq \frac{1}{2} N T_{\varepsilon}$. Define inductively three sequences by

$$
\begin{aligned}
\sigma_{k} & =\tau_{0}+\sum_{i=0}^{k-1} s_{i} \\
\tau_{k} & =\tau_{k-1}+s_{k-1}+N T_{\varepsilon}=\sigma_{k}+k N T_{\varepsilon} \\
s_{k} & =-\eta\left(\tau_{k}\right) a_{1} .
\end{aligned}
$$

By the Claim 3 we get by induction $\eta\left(\tau_{k}\right) \leq 2^{-k} \eta\left(\tau_{0}\right)$ and $\left|s_{k}\right| \leq 2^{-k-1} N T_{\varepsilon}$. Hence there exists the limit $\sigma=\lim \sigma_{k} \leq \tau_{0}+N T_{\varepsilon}$ and then $\tau_{k} \rightarrow \infty$ as $k \rightarrow \infty$.

We claim $\sigma$ is the correct translation parameter. In fact, choose $k$ such that $t=k N T_{\varepsilon}+[t]$ with $[t] \in I_{N}$, and write

$$
\begin{aligned}
w_{i, \sigma}(t, \theta) & =v_{i}(t+\sigma, \theta)-\Lambda_{i} v_{\varepsilon}(t) \\
& =v_{i}(t+\sigma, \theta)-v_{i}\left(t+\sigma_{k}, \theta\right)+v_{i}\left(t+\sigma_{k}, \theta\right)-\Lambda_{i} v_{\varepsilon}(t) .
\end{aligned}
$$

Since $\partial_{t} v_{i}$ is uniformly bounded, we have

$$
v_{i}(t+\sigma, \theta)-v_{i}\left(t+\sigma_{k}, \theta\right)=\partial_{t} v_{i}\left(t_{0}\right) \sum_{j=k}^{\infty} s_{j}=O\left(2^{-k}\right),
$$

for some $t_{0}$. Besides,

$$
v_{i}\left(t+\sigma_{k}, \theta\right)-\Lambda_{i} v_{\varepsilon}(t)=v_{i}\left(\tau_{k}+[t], \theta\right)-\Lambda_{i} v_{\varepsilon}([t])=w_{i, \tau_{k}}([t], \theta) .
$$

Thus,

$$
\mathcal{W}_{\sigma}(t, \theta)=\mathcal{W}_{\tau_{k}}([t], \theta)+O\left(2^{k}\right)
$$


Since $b \max _{I_{N}}\left|\mathcal{W}_{\tau_{k}}\right|=\eta\left(\tau_{k}\right) \leq \eta\left(\tau_{k}\right) \leq 2^{-k} \eta\left(\tau_{0}\right)$, it holds $\left|w_{i, \sigma}(t, \theta)\right|=O\left(2^{-k}\right)$ or equivalently, using that $t=k N T_{\varepsilon}+[t]$, we have

$$
\left|w_{i, \sigma}(t, \theta)\right| \leq C_{1} e^{-\frac{\log 2}{N T_{\varepsilon}} t}
$$

which finishes the proof of the theorem. 


\section{Chapter 4}

\section{Yamabe-type system in the punctured ball}

Let $\mathrm{g}$ be a smooth Riemannian metric on the unit ball $B_{1}^{n}(0) \subset \mathbb{R}^{n}, n \geq 3$. Consider a positive solution to the system

$$
\Delta_{g} u_{i}-\sum_{j=1}^{2} A_{i j}(x) u_{j}+\frac{n(n-2)}{4}|\mathcal{U}|^{\frac{4}{n-2}} u_{i}=0
$$

in the punctured ball $\Omega=B_{1}^{n}(0) \backslash\{0\}$ and $\mathcal{U}=\left(u_{1}, u_{2}\right)$. In order to prove our main result, during the proof of the results we will need sometimes that the potential $A$ satisfy some of the following conditons.

(H1) $-A$ is cooperative, that is, the components in the nondiagonal $A_{i j}$ of $A, i \neq j$, are nonpositive;

(H2) In dimension $n=5$, there exists a $C^{2}$-function $f$ such that

$$
A(x)=f(x) I d_{2}+O(|x|)
$$

near the origin, where $I d_{2}$ is the identity matrix.

\subsection{Upper bound near a singularity}

In this section we will obtain upper and lower bounds for solutions to our system defined in the punctured ball. Considering the diffeomorphism $\Phi(t, \theta)=e^{-t} \theta$ between 
the half cylinder and the punctured ball introduced in the first chapter, define the change of variables from Fowler as

$$
v_{i}(t, \theta)=|x|^{\frac{n-2}{2}} u_{i}(x)
$$

and $\hat{g}=e^{2 t} \Phi^{*} g=\left(e^{\frac{n-2}{2} t}\right)^{\frac{4}{n-2}} \Phi^{*} g$. Using that

$$
L_{v^{\frac{4}{n-2} g}}(u)=v^{-\frac{n+2}{n-2}} L_{g}(v u)
$$

where the linear operator $L_{g}=\Delta_{g}-\frac{n-2}{4(n-1)} R_{g}$ is the conformal laplacian, we obtain that the system is equivalent to

$$
\mathcal{L}_{\hat{g}}\left(v_{i}\right)-\sum_{j=1}^{2} B_{i j} v_{j}+\frac{n(n-2)}{4}|\mathcal{V}|^{\frac{4}{n-2}} v_{i}=0,
$$

where

$$
\begin{array}{r}
\mathcal{L}_{\hat{g}}\left(v_{i}\right)=\Delta_{\hat{g}} v_{i}-\frac{n-2}{4(n-1)}\left(R_{\hat{g}}-e^{-2 t} R_{\Phi^{*} g}\right) v_{i}, \\
B_{i j}=e^{-2 t} A_{i j} \circ \Phi
\end{array}
$$

and $\mathcal{V}=\left(v_{1}, v_{2}\right)$

It is also useful to remember that in cylindrical coordinates we have

$$
R_{\hat{g}}=(n-2)(n-1)+2(n-1) e^{-t} \frac{\partial_{r} \sqrt{|g|}}{\sqrt{|g|}}+e^{-2 t} R_{g} \circ \Phi,
$$

and so

$$
R_{\hat{g}}-e^{-2 t} R_{\Phi^{*} g}=(n-2)(n-1)+2(n-1) e^{-t} \frac{\partial_{r} \sqrt{|g|}}{\sqrt{|g|}} \circ \Phi .
$$

Theorem 4.1 Suppose $3 \leq n \leq 5$. Assume that $\mathcal{U}=\left(u_{1}, u_{2}\right)$ is a positive solution of (1) in $\Omega=B_{1}^{n}(0) \backslash\{0\}$. There exists a constant $c>0$ such that

$$
|\mathcal{U}|(x) \leq c d_{g}(x, 0)^{\frac{2-n}{2}}
$$

for $0<d_{g}(x, 0)<\frac{1}{2}$.

Proof. Given $x_{0} \in \Omega$ with $d_{g}\left(x_{0}, 0\right)<\frac{1}{2}$ and $s \in\left(0, \frac{1}{4}\right)$ such that $\bar{B}_{s}\left(x_{0}\right) \subset \Omega$, define

$$
f(x)=\left(s-d_{g}\left(x, x_{0}\right)\right)^{\frac{n-2}{2}}|\mathcal{U}(x)|
$$

for $x \in \bar{B}_{s}\left(x_{0}\right)$. It suffices to show that there exists a positive constant $C$ such that any such $f$ satisfies $f(x) \leq C$ in $B_{s}\left(x_{0}\right)$. To see this, taking $s=\frac{\left|x_{0}\right|}{2}$ gives $f\left(x_{0}\right)=$ $s^{\frac{n-2}{2}}\left|\mathcal{U}\left(x_{0}\right)\right| \leq C$. 
The proof will be by contradiction, so assume there is no such constant $C$. Then we can find a sequence of points $x_{0, k}$ and positive numbers $s_{k}$ so that, if $x_{1, k}$ denotes the maximum point of the corresponding $f_{k}$, we have

$$
f_{k}\left(x_{1, k}\right)=\left(s_{k}-d_{g}\left(x, x_{0, k}\right)\right)^{\frac{n-2}{2}}\left|\mathcal{U}\left(x_{1, k}\right)\right| \rightarrow \infty
$$

Note that, $0<s_{k}<\frac{1}{4}$ implies that $\left(s_{k}-d_{g}\left(x, x_{0, k}\right)\right)^{\frac{n-2}{2}}<2^{2-n}$ and therefore $2^{n-2} f_{k}(x)<$ $|\mathcal{U}(x)|$. Hence $\left|\mathcal{U}\left(x_{1, k}\right)\right| \rightarrow \infty$ and consequently $x_{1, k} \rightarrow 0$.

Let $\varepsilon_{k}=\left|\mathcal{U}\left(x_{1, k}\right)\right|^{\frac{-2}{n-2}}$ and define,

$$
\tilde{u}_{i, k}(y)=\varepsilon_{k}^{\frac{n-2}{2}} u_{i}\left(\exp _{x_{1, k}}\left(\varepsilon_{k} y\right)\right)
$$

for all $i \in\{1,2\}$. Note that $\left|\tilde{\mathcal{U}}_{k}(0)\right|=1$, where $\tilde{\mathcal{U}}_{k}=\left(\tilde{u}_{1, k}, \tilde{u}_{2, k}\right)$. Also note that the function $\tilde{u_{i, k}}$ is defined for all $y$ such that $|y| \leq \varepsilon_{k}^{-1}\left(s_{k}-d_{g}\left(x_{0, k}, x_{1, k}\right)\right)$. Moreover, if

$$
d_{g}\left(x, x_{1, k}\right) \leq r_{k}=\frac{1}{2}\left(s_{k}-d_{g}\left(x_{0, k}, x_{1, k}\right)\right)
$$

then

$$
d_{g}\left(x, x_{0, k}\right)-d_{g}\left(x_{0, k}, x_{1, k}\right) \leq d_{g}\left(x, x_{1, k}\right) \leq r_{i}=\frac{1}{2}\left(s_{k}-d_{g}\left(x_{0, k}, x_{1, k}\right)\right),
$$

and this implies that

$$
d_{g}\left(x, x_{0, k}\right) \leq s_{k}+\frac{1}{2}\left(-s_{k}+d_{g}\left(x_{0, k}, x_{1, k}\right)\right) .
$$

Thus

$$
r_{k} \leq s_{k}-d_{g}\left(x_{0, k}, x_{1, k}\right)
$$

Therefore

$$
r_{k}^{\frac{n-2}{2}}|\mathcal{U}(x)| \leq f_{k}(x) \leq f_{k}\left(x_{1, k}\right)=\left(2 r_{k} \varepsilon_{k}^{-1}\right)^{\frac{n-2}{2}}=\left(2 r_{k}\right)^{\frac{n-2}{2}}\left|\mathcal{U}\left(x_{1, k}\right)\right| \rightarrow \infty
$$

and

$$
|\mathcal{U}(x)| \leq 2^{\frac{n-2}{2}}\left|\mathcal{U}\left(x_{1, k}\right)\right|
$$

for all $x$ with $d_{g}\left(x, x_{1, k}\right) \leq r_{k}$. It follows that

$$
\tilde{u}_{i, k}(y)=\left|\mathcal{U}\left(x_{1, k}\right)\right|^{-1} u_{i}\left(\exp _{x_{1, k}}\left(\varepsilon_{k} y\right)\right) \leq\left|\mathcal{U}\left(x_{1, k}\right)\right|^{-1}|\mathcal{U}(x)| \leq 2^{\frac{n-2}{2}},
$$

for all $y$ with $\left|\varepsilon_{k} y\right| \leq r_{k}$, that is, $|y| \leq r_{k} \varepsilon_{k}^{-1} \rightarrow \infty$. 
Now if we define $\left(\tilde{g}_{k}\right)_{l m}(y):=g_{l m}\left(\varepsilon_{k} y\right)$, then $\tilde{u}_{i, k}$ satisfies

$$
\Delta_{\tilde{g}_{k}} \tilde{u}_{i, k}(y)-\varepsilon_{k}^{2} \sum_{j=1}^{2} \tilde{A}_{i j}^{k}(y) \tilde{u}_{j, k}(y)+\frac{n(n-2)}{4}\left|\tilde{\mathcal{U}}_{k}(y)\right|^{\frac{4}{n-2}} \tilde{u}_{i, k}(y)=0,
$$

where $\tilde{A}_{i j}^{k}(y):=A_{i j}\left(\exp _{x_{1, k}}\left(\varepsilon_{k} y\right)\right)$.

Standard elliptic theory then implies that, after passing to a subsequence, $\left\{\tilde{u}_{i, k}\right\}_{k}$ converge in $C^{2}$ norm on compact subsets of $\mathbb{R}^{n}$ to a positive solution $u_{i, 0}$ to

$$
-\Delta u_{i, 0}=\frac{n(n-2)}{4}\left|\mathcal{U}_{0}\right|^{\frac{4}{n-2}} u_{i, 0}
$$

which satisfies $\left|\mathcal{U}_{0}(0)\right|=1$ and $u_{i, 0}(y) \leq 2^{\frac{n-2}{2}}$ for every $y \in \mathbb{R}^{n}$. By a theorem due to Druet and Hebey [6], we can conclude that there exist $a \in \mathbb{R}^{n}, \mu>0$ and $\Lambda \in \mathbb{S}_{+}^{1}$ such that

$$
\mathcal{U}_{0}(y)=\left(\frac{2 \mu}{1+\mu^{2}|y-a|^{2}}\right)^{\frac{n-2}{2}} \Lambda .
$$

Since $\left|\mathcal{U}_{0}(0)\right|=1$, we conclude that $|a| \leq 1$ and $\mu \in[1 / 2,1]$.

Now note that $\mathcal{U}_{0}$ has a nondegenerate maximum point at $a$. Then we conclude that there is a sequence $y_{k} \rightarrow a$ such that $y_{k}$ is a nondegenerate maximum point of $\left|\tilde{\mathcal{U}}_{k}\right|$. We can assume $\left|y_{k}\right| \leq 2$ and therefore there will be a corresponding local maximum point $x_{2, k}$ of $|\mathcal{U}|$ satisfying $d_{g}\left(x_{2, k}, x_{1, k}\right) \leq 2 \varepsilon_{k}$. If we redefine the functions $\tilde{u}_{i, k}$ replacing $x_{1, k}$ by $x_{2, k}$ we get as before that a subsequence $\left\{\tilde{u}_{i, k}\right\}_{k}$ converge in the $C^{2}$ norm on compact subsets of $\mathbb{R}^{n}$ to

$$
\mathcal{U}_{0}(y)=\left(\frac{1}{1+\frac{1}{4}|y|^{2}}\right)^{\frac{n-2}{2}} \Lambda
$$

Note that, by construction, we have that $\left|x_{2, k}\right|<7 / 8$, so we can consider $\tilde{u}_{i, k}$ as defined for $|y| \leq \frac{1}{16} \varepsilon_{k}^{-1}$, with a possible singularity at some point on the sphere of radius $\left|x_{2, k}\right| \varepsilon_{k}^{-1} \rightarrow \infty$, where now $\varepsilon_{k}=\left|\mathcal{U}\left(x_{2, k}\right)\right|^{-\frac{2}{n-2}}$.

Let us introduce

$$
v_{i, k}(t, \theta)=|y|^{\frac{n-2}{2}} \tilde{u}_{i, k}(y)
$$

where $t=-\log |y|$ and $\theta=\frac{y}{|y|}$. These functions are defined for $t>\log \left(\frac{1}{16} \varepsilon_{k}^{-1}\right)$, with a singularity at some point $\left(t_{k}^{\prime}, \theta_{k}^{\prime}\right), t_{k}^{\prime}=\log \left(\left|x_{2, k} \varepsilon_{k}^{-1}\right|\right)$. Now define,

$$
\mathcal{V}_{0}(t)=|y|^{\frac{n-2}{2}} \mathcal{U}_{0}(y)=\left(e^{t}+\frac{1}{4} e^{-t}\right)^{\frac{2-n}{2}} \Lambda \text {. }
$$


Since $\tilde{\mathcal{U}}_{k} \rightarrow \tilde{\mathcal{U}}_{0}$ in the $C_{\text {loc }}^{2}$ topology, we know that given $R>0$ the inequalities

$$
\begin{array}{r}
\left|\mathcal{V}_{k}(t, \theta)-\mathcal{V}_{0}(t)\right| \leq R^{-1} e^{\frac{2-n}{2} t} \\
\left|\partial_{t} \mathcal{V}_{k}(t, \theta)-\mathcal{V}_{0}^{\prime}(t)\right| \leq R^{-1} e^{\frac{2-n}{2} t} \\
\left|\partial_{t}^{2} \mathcal{V}_{k}(t, \theta)-\mathcal{V}_{0}^{\prime \prime}(t)\right| \leq R^{-1} e^{\frac{2-n}{2} t} \\
\left|\partial_{\theta_{l}} \mathcal{V}_{k}(t, \theta)\right| \leq R^{-1} e^{\frac{2-n}{2} t} \\
\left|\partial_{\theta_{l} \theta_{m}}^{2} \mathcal{V}_{k}(t, \theta)\right| \leq R^{-1} e^{\frac{2-n}{2} t}
\end{array}
$$

are satisfied for $t>-\log R$ and sufficiently large $k$.

In particular

$$
\partial_{t} v_{i, k}(-\log 3 n, \theta)=\frac{2-n}{2}\left((3 n)^{-1}+\frac{3 n}{4}\right)^{\frac{2-n}{2}}-R^{-1}(3 n)^{\frac{2-n}{2}}>0
$$

for all $\theta \in \mathbb{S}^{n-1}$ and for $R>0$ large enough.

For a fixed small number $\delta>0$ to be chosen later, define

$$
\Gamma_{k}=\left[\log \left(\delta \varepsilon_{k}^{-1}\right), \infty\right) \times \mathbb{S}^{n-1}
$$

Since $\tilde{\mathcal{U}}_{k} \rightarrow \mathcal{U}_{0}$ in the $C_{\text {loc }}^{2}$ topology and

$$
\mathcal{V}_{0}\left(\log \left(\delta \varepsilon_{k}^{-1}\right)\right)=\left(\delta^{-1} \varepsilon_{k}+\frac{\delta \varepsilon_{k}^{-1}}{4}\right)^{\frac{2-n}{2}} \Lambda \geq c(\delta)>0
$$

we obtain

$$
\mathcal{V}_{k}\left(\log \left(\delta \varepsilon_{k}^{-1}\right)\right) \geq c(\delta)>0
$$

We will apply the Alexandrov technique to $v_{i, k}$ on the region $\Gamma_{k}$ reflecting across the spheres $\{\lambda\} \times S^{n-1}$. To simplify the notation we will drop the subscript $k$.

Define $\Gamma_{\lambda}=\left[-\log \left(\delta \varepsilon^{-1}\right), \lambda\right], \hat{g}_{\lambda}$ the pull-back of the metric $\hat{g}$ by the reflection across the sphere $\{\lambda\} \times S^{n-1}$

$$
\begin{aligned}
& v=v_{1}+v_{2} \\
& v_{i, \lambda}(t, \theta)=v_{i}(2 \lambda-t, \theta)
\end{aligned}
$$

and

$$
v_{\lambda}(t)=v(2 \lambda-t, \theta)
$$

Remember that $\hat{g}=e^{2 t} \Phi^{*} \tilde{g}$, and $\left(\tilde{g}_{k}\right)_{l m}(y)=g_{l m}\left(\varepsilon_{k} y\right)$. 
By (4.4) we can write

$$
\begin{gathered}
\mathcal{L}_{\hat{g}}\left(v_{i}-v_{i, \lambda}\right)-\sum_{j=1}^{2} B_{i j}\left(v_{j}-v_{j, \lambda}\right)+\frac{n(n-2)}{4}|\mathcal{V}|^{\frac{4}{n-2}} v_{i}-\frac{n(n-2)}{4}\left|\mathcal{V}_{\lambda}\right|^{\frac{4}{n-2}} v_{i, \lambda} \\
=\left(\mathcal{L}_{\hat{g}_{\lambda}}-\mathcal{L}_{\hat{g}}\right) v_{i, \lambda}-\sum_{j=1}^{2}\left(B_{i j}^{\lambda}-B_{i j}\right) v_{j, \lambda} .
\end{gathered}
$$

Note that,

$$
|\mathcal{V}|^{\frac{4}{n-2}} v-\left|\mathcal{V}_{\lambda}\right|^{\frac{4}{n-2}} v_{\lambda}=\sum_{j=1}^{2} b_{i, \lambda}\left(v_{i}-v_{i, \lambda}\right)
$$

where

$$
b_{i, \lambda}=v\left(v_{i}+v_{i, \lambda}\right) \frac{|\mathcal{V}|^{\frac{4}{n-2}}-\left|\mathcal{V}_{\lambda}\right|^{\frac{4}{n-2}}}{|\mathcal{V}|^{2}-\left|\mathcal{V}_{\lambda}\right|^{2}}+\left|\mathcal{V}_{\lambda}\right|^{\frac{4}{n-2}}>0
$$

By 4.10) we have

$$
\mathbb{L}_{\hat{g}}\left(v_{1}-v_{1, \lambda}, v_{2}-v_{2, \lambda}\right)+\sum_{i=1}^{2} b_{i, \lambda}\left(v_{i}-v_{i, \lambda}\right)=Q_{\lambda},
$$

where

$$
Q_{\lambda}=\left(\mathcal{L}_{\hat{g}_{\lambda}}-\mathcal{L}_{\hat{g}}\right) v_{\lambda}+\sum_{i, j=1}^{2}\left(B_{i j}^{\lambda}-B_{i j}\right) v_{j, \lambda}
$$

and

$$
\mathbb{L}_{\hat{g}}\left(w_{1}, w_{2}\right)=\mathcal{L}_{\hat{g}}\left(\sum_{j=1}^{2} w_{j}\right)-\sum_{i, j=1}^{2} B_{i j} w_{j} .
$$

Claim 1: There exists a constant $c_{1}>0$, not depending on $\delta$, such that $\left|Q_{\lambda}(t, \theta)\right| \leq$ $q_{\lambda}(t)=c_{1} \varepsilon^{2} e^{\frac{n-6}{2} t} e^{(2-n) \lambda}$.

First, we observe that $\frac{\partial_{r} \sqrt{|g|}}{\sqrt{|g|}}=O(|x|)$ and $v_{\lambda}(t)=O\left(e^{\frac{2-n}{2}(2 \lambda-t)}\right)$ and this implies that

$$
\begin{aligned}
\left|R_{\hat{g}_{\lambda}}-e^{-2 t} R_{\Phi^{*} \tilde{g}_{\lambda}}-\left(R_{\hat{g}}-e^{-2 t} R_{\Phi^{*} \tilde{g}}\right)\right| v_{\lambda}(t, \theta) & \leq C \varepsilon^{2} e^{-2 t} e^{\frac{2-n}{2}(2 \lambda-t)} \\
& =C \varepsilon^{2} e^{\frac{n-6}{2} t} e^{(2-n) \lambda} .
\end{aligned}
$$

On the other hand, since $\hat{g}=e^{2 t} \Phi^{*} \tilde{g}$ and $\tilde{g}_{i j}=\delta_{i j}+O\left(\varepsilon^{2}|y|^{2}\right)$ in normal coordinates, we have $\hat{g}=d t^{2}+d \theta^{2}+O\left(\varepsilon^{2} e^{-2 t}\right)$. It follows that

$$
\left|\left(\Delta_{\hat{g}_{\lambda}}-\Delta_{\hat{g}}\right) v_{\lambda}\right| \leq C \varepsilon^{2} e^{\frac{n-6}{2} t} e^{(2-n) \lambda} .
$$

Also by 4.5 we have

$$
\left|\sum_{i, j=1}^{2}\left(B_{i j}^{\lambda}-B_{i j}\right) v_{j, \lambda}\right| \leq C \varepsilon^{2} e^{\frac{n-6}{2} t} e^{(2-n) \lambda},
$$


proving the first claim.

Claim 2: Suppose $3 \leq n \leq 5$, and let $\gamma>0$ be a small number. Then there exists a family of functions $h_{\lambda}(t)$, defined on $\Gamma_{\lambda}$, satisfying the following properties

$$
\begin{array}{r}
h_{\lambda}(\lambda)=0 ; \\
h_{\lambda} \geq 0 ; \\
\mathbb{L}_{\hat{g}}\left(h_{\lambda}, h_{\lambda}\right) \geq Q_{\lambda} ;
\end{array}
$$

$$
h_{\lambda} \leq v_{i}-v_{i, \lambda} \text {, if } \lambda \text { is sufficiently large }
$$

and

$$
h_{\lambda}\left(-\log \left(\delta \varepsilon^{-1}\right)\right) \leq c_{3} \max \left\{\varepsilon^{\frac{n-2}{2}}, \varepsilon^{\frac{6-n}{2}-\gamma}\right\}
$$

for some positive constant $c$ which depends only on $\delta$.

We will begin the proof of the second claim with some useful computations for the operator $\mathbb{L}_{\hat{g}}$ in order to get the desired estimates. Remember that $\hat{g}=e^{\frac{2-n}{2} t} \Phi^{*} \tilde{g}$, thus by 4.3 we obtain

$$
L_{\hat{g}} f=e^{-\frac{n+2}{2} t} L_{\Phi^{*} \tilde{g}}\left(e^{\frac{n-2}{2} t} f\right)=e^{-\frac{n+2}{2} t}\left(\Delta_{\tilde{g}}\left(|y|^{\frac{2-n}{2}} \tilde{f}\right)-\frac{n-2}{4(n-1)}|y|^{\frac{2-n}{2}} R_{\tilde{g}} \tilde{f}\right) \circ \Phi,
$$

where $\tilde{f}=f \circ \Phi^{-1}$. Hence

$$
\mathcal{L}_{\hat{g}} f=L_{\hat{g}} f+\frac{n-2}{4(n-1)} e^{-2 t} R_{\Phi^{*} g} f=e^{-\frac{n+2}{2} t} \Delta_{\tilde{g}}\left(|y|^{\frac{2-n}{2}} \tilde{f}\right) \circ \Phi .
$$

So, if $f$ depends only on $t$, then we can use the expression of the laplacian for radial functions given by

$$
\Delta_{\tilde{g}} u=\partial_{r}^{2} u+\frac{n-1}{r} \partial_{r}+\frac{\partial_{r} \sqrt{|\tilde{g}|}}{\sqrt{|\tilde{g}|}} \partial_{r} u .
$$

Note that

$$
\partial_{r}\left(|y|^{\frac{2-n}{2}} \tilde{f}\right)=\frac{2-n}{2}|y|^{-\frac{n}{2}} \tilde{f}+|y|^{\frac{2-n}{2}} \partial_{r} \tilde{f}
$$

and

$$
\partial_{r}^{2}\left(|y|^{\frac{2-n}{2}} \tilde{f}\right)=\frac{n(n-2)}{4}|y|^{-\frac{n+2}{2}} \tilde{f}+(2-n)|y|^{-\frac{n}{2}} \partial_{r} \tilde{f}+|y|^{\frac{2-n}{2}} \partial_{r}^{2} \tilde{f} .
$$

On the other hand, since $\tilde{f}(|y|)=f(-\log |y|)$, we have

$$
\partial_{r} \tilde{f}=-|y|^{-1} f^{\prime} \circ \Phi^{-1}
$$


and

$$
\partial_{r}^{2} \tilde{f}=|y|^{-2} f^{\prime} \circ \Phi^{-1}+|y|^{-2} f^{\prime \prime} \circ \Phi^{-1}
$$

Then we get

$$
\partial_{r}\left(|y|^{\frac{2-n}{2}} \tilde{f}\right)=\frac{2-n}{2}|y|^{-\frac{n}{2}} f \circ \Phi^{-1}-|y|^{\frac{n}{2}} f^{\prime} \circ \Phi^{-1}
$$

and

$$
\partial_{r}^{2}\left(|y|^{\frac{n-2}{2}} \tilde{f}\right)=\frac{n(n-2)}{4}|y|^{-\frac{n+2}{2}} f \circ \Phi^{-1}+(n-1)|y|^{-\frac{n+2}{2}} f^{\prime} \circ \Phi^{-1}+|y|^{-\frac{n+2}{2}} f^{\prime \prime} \circ \Phi^{-1}
$$

Thus

$$
\begin{gathered}
\Delta_{\tilde{g}}\left(|y|^{-\frac{n+2}{2}} \tilde{f}\right)=|y|^{-\frac{n+2}{2}} f^{\prime \prime} \circ \Phi^{-1}-\left(\frac{n-2}{2}\right)^{2}|y|^{-\frac{n+2}{2}} f \circ \Phi^{-1}+O\left(\varepsilon^{2}|y|^{\frac{2-n}{2}}\right) f \circ \Phi^{-1} \\
+O\left(\varepsilon^{2}|y|^{\frac{2-n}{2}}\right) f^{\prime} \circ \Phi^{-1}
\end{gathered}
$$

which implies that

$$
e^{-\frac{n+2}{2} t} \Delta_{\tilde{g}}\left(|y|^{\frac{2-n}{2}} \tilde{f}\right) \circ \Phi=f^{\prime \prime}+O\left(\varepsilon^{2} e^{-2 t}\right) f^{\prime}-\left(\left(\frac{n-2}{2}\right)^{2}+O\left(\varepsilon^{2} e^{-2 t}\right)\right) f .
$$

Therefore,

$$
\mathcal{L}_{\hat{g}} f=f^{\prime \prime}+O\left(\varepsilon^{2} e^{-2 t}\right)-f^{\prime}\left(\left(\frac{n-2}{2}\right)^{2}+O\left(\varepsilon^{2} e^{-2 t}\right)\right) f,
$$

for any function $f$ that depends only on $t$. Besides by 4.5 we obtain

$$
B_{i j}=O\left(\varepsilon^{2} e^{-2 t}\right)
$$

Thus, if $f=f_{1}+f_{2}$, then

$$
\mathbb{L}_{\widehat{g}}\left(f_{1}, f_{2}\right)=f^{\prime \prime}+O\left(\varepsilon^{2} e^{-2 t}\right) f^{\prime}-\left(\left(\frac{n-2}{2}\right)^{2}+O\left(\varepsilon^{2} e^{-2 t}\right)\right) f+O\left(\varepsilon^{2} e^{-2 t}\right) f .
$$

Given a small number $\gamma>0$, consider the linear operator $\bar{L}$ given by

$$
\bar{L}(f)=f^{\prime \prime}+\gamma f^{\prime}-\left(\left(\frac{n-2}{2}\right)^{2}+\gamma\right) f .
$$

and let $\gamma_{1}=\frac{8-n}{2} \gamma>0$ and $a(n)=\frac{1}{2(4-n)-\gamma_{1}}$.

We know proceed to the construction of the auxiliary functions. Define

$$
h_{\lambda}(t)=\frac{a(n)}{2} c_{1} \varepsilon^{2} e^{(2-n) \lambda} e^{\frac{n-6}{2} t}\left(1-e^{\left(4-n-\gamma_{2}\right)(t-\lambda)}\right),
$$


where $\gamma_{2}>0$ is chosen such that the function $e^{\left(\frac{2-n}{2}-\gamma_{2}\right) t}$ is in the kernel of 4.19. Observe that we can make $\gamma_{1}$ and $\gamma_{2}$ as small as necessary depending on $\gamma$.

Note that the first two properties follows directly from the definition and moreover $h_{\lambda}^{\prime} \leq 0$ in $(-\infty, \lambda]$. Since $t \geq-\log \left(\delta \varepsilon^{-1}\right)$ it holds $\varepsilon^{2} e^{-2 t} \leq \delta^{2}$. Choosing $\delta^{2}<<\gamma$, if $f \geq 0$ and $f_{i}^{\prime} \leq 0$, then by 4.18 and 4.19 we get

$$
\left.\mathbb{L}_{\hat{g}}\left(f_{1}, f_{2}\right) \geq f^{\prime \prime}+\gamma f^{\prime}-\left(\left(\frac{n-2}{2}\right)^{2}+\gamma\right)\right) f=\bar{L}(f) .
$$

Now observe that

$$
h_{\lambda}(t)=a(n) c_{1} \varepsilon^{2} e^{(2-n) \lambda}\left(e^{\frac{n-6}{2} t}-e^{\left(\frac{2-n}{2}-\gamma_{2}\right) t} e^{\left(4-n-\gamma_{2}\right) \lambda}\right)
$$

and

$$
\begin{array}{r}
\bar{L}\left(h_{\lambda}\right)=a(n) c_{1} \varepsilon^{2} e^{(2-n) \lambda}\left(\left(\frac{n-6}{2}\right)^{2}+\gamma \frac{n-6}{2}-\left(\frac{n-2}{2}\right)^{2}-\gamma\right) e^{\frac{n-6}{2} t} \\
=a(n) c_{1} \varepsilon^{2} e^{(2-n) \lambda}\left(2(4-n)+\gamma \frac{n-8}{2}\right) e^{\frac{n-6}{2} t}=q_{\lambda}(t) .
\end{array}
$$

Therefore

$$
\mathbb{L}_{\hat{g}}\left(h_{\lambda}, h_{\lambda}\right) \geq \bar{L}\left(h_{\lambda}\right)=q_{\lambda} \geq\left|Q_{\lambda}\right|
$$

which proves (4.15). Besides,

$$
h_{\lambda}\left(-\log \left(\delta \varepsilon^{-1}\right)\right)=a(n) c_{1}\left(\delta^{\frac{6-n}{2}} e^{(2-n) \lambda} \varepsilon^{\frac{n-2}{2}}-\delta^{\frac{n-2}{2}+\gamma_{2}} e^{\left(-2+\gamma_{2}\right) \lambda} \varepsilon^{\frac{6-n}{2}-\gamma_{2}}\right),
$$

gives us the estimate 4.17).

Since $h_{\lambda}^{\prime} \leq 0$, by 4.21 we obtain that

$$
\max _{\Gamma_{\lambda}} h_{\lambda} \rightarrow 0 \text { as } \lambda \rightarrow \infty
$$

For sufficiently large $t_{0}$ consider the set $\Gamma_{t_{0}}=\left[-\log \left(\delta \varepsilon^{-1}\right), t_{0}\right] \times \mathbb{S}^{n-1}$. Consequently we have that

$$
v_{i, \lambda}(t) \leq C e^{\frac{2-n}{2}(2 \lambda-t)}
$$

forasmuch as $v_{i}=O\left(e^{\frac{n-2}{2} t}\right)$. Define

$$
w_{i, \lambda}=v_{i}-v_{i, \lambda}-h_{\lambda} \geq 0
$$

By 4.22 it follows that $w_{i, \lambda} \geq 0$ on $\Gamma_{t_{0}}$ for sufficiently large $\lambda$. Let us show that $w_{i, \lambda} \geq 0$ for all $t \in\left[t_{0}, \lambda\right]$. A direct computation gives

$$
h_{\lambda}^{\prime}(t)=\frac{n-6}{4} a(n) c_{1} \varepsilon e^{(2-n) \lambda} e^{\frac{n-6}{2} t}\left(1-\frac{2}{n-6}\left(\frac{2-n}{2}-\gamma_{2}\right) e^{\left(4-n-\gamma_{2}\right)(t-\lambda)}\right) .
$$


Hence, for all $t \in\left[t_{0}, \lambda\right]$, we have

$$
\left|h_{\lambda}^{\prime}(t)\right| \leq C\left(\delta, \varepsilon, t_{0}\right)\left(e^{(2-n) \lambda}+e^{\left(-2+\gamma_{2}\right) \lambda}\right) .
$$

Now

$$
\partial_{t} w_{i, \lambda}(t, \theta)=\partial_{t} v_{i}(t, \theta)+\partial_{t} v_{i}(2 \lambda-t, \theta)-h_{\lambda}^{\prime}(t) .
$$

Thus, because of the previous estimates and (4.9), when $t \in\left[t_{0}, \lambda\right]$, we get

$$
\begin{array}{r}
\partial_{t} w_{i, \lambda}(t, \theta) \leq-C e^{\frac{2-n}{2} t}-C e^{\frac{2-n}{2}(2 \lambda-t)}-C\left(\delta, \varepsilon, t_{0}\right)\left(e^{(2-n) \lambda}+e^{\left(-2+\gamma_{2}\right) \lambda}\right) \\
\leq-C e^{\frac{2-n}{\lambda}}<0,
\end{array}
$$

for $t_{0}$ and $\lambda$ sufficiently large. Since $w_{i, \lambda}(\lambda, \theta)=0$ for all $\theta \in \mathbb{S}^{n-1}$, we conclude that $w_{i, \lambda} \geq 0$ for all $t \in\left[t_{0}, \lambda\right]$, for sufficiently large $\lambda$. This finishes the proof of the Claim 2 .

Once we proved the previous claim, using the same notation, we note that

$$
w_{i, \lambda}(\lambda, \theta)=0, \text { for all } \theta \in \mathbb{S}^{n-1}
$$

and

$$
\mathbb{L}_{\hat{g}}\left(w_{1, \lambda}, w_{2, \lambda}\right)+\sum_{i=1}^{2} b_{i, \lambda} w_{i, \lambda}=Q_{\lambda}-\mathbb{L}_{\hat{g}}\left(h_{\lambda}, h_{\lambda}\right) \leq 0 .
$$

In order to conclude the proof of Theorem 4.1, we will also need the following:

Claim 3: There exist $\lambda_{0}>-\log (3 n)$ and $\theta_{0} \in \mathbb{S}^{n-1}$ such that

$$
w_{\lambda_{0}}\left(-\log \left(\delta \varepsilon^{-1}\right), \theta_{0}\right)=0 \text {. }
$$

Define

$$
\lambda_{0}=\inf \left\{\lambda_{1} ; w_{\lambda}(t, \theta) \geq 0 \text { in } \Gamma_{\lambda}, \forall \lambda \geq \lambda_{1}\right\} .
$$

Note that 4.16) implies that this set is not empty. Besides, if we take $\lambda=-\log (3 n)$, then by 4.9 and 4.23 we get

$$
\partial_{t} w_{\lambda}(-\log (3 n), \theta)=2 \partial_{t} v(-\log (3 n), \theta)-2 h_{\lambda}^{\prime}(-\log (3 n))>0 .
$$

Moreover since $w_{\lambda}(-\log (3 n), \theta)=0$, we have $\lambda_{0}>-\log (3 n)$.

By continuity, $w_{\lambda_{0}} \geq 0$ in $\Gamma_{\lambda_{0}}$. Suppose the claim is false. Then $w_{\lambda_{0}}\left(-\log \left(\delta \varepsilon^{-1}\right), \theta\right)>$ 0 for all $\theta \in \mathbb{S}^{n-1}$. We notice that by, 4.12, 4.5 and 4.15) we can apply the Maximum Principle, since for $\varepsilon>0$ small enough we have

$$
\Delta_{\hat{g}} w_{\lambda_{0}}-D w_{\lambda_{0}} \leq \mathbb{L}_{\hat{g}}\left(w_{1, \lambda_{0}}, w_{2, \lambda_{0}}\right)=Q_{\lambda_{0}}-\mathbb{L}_{\hat{g}}\left(h_{\lambda_{0}}, h_{\lambda_{0}}\right)-\sum_{i=1}^{2} b_{i, \lambda} w_{i, \lambda_{0}} \leq 0
$$


where $D$ is a positive function. This implies that $w_{\lambda_{0}}(t, \theta)>0$ for every $-\log \left(\delta \varepsilon^{-1}\right)<$ $t<\lambda_{0}$ and $\theta \in \mathbb{S}^{n-1}$, since on the boundary $\partial \Gamma_{\lambda_{0}}$ we have $w_{\lambda_{0}} \geq 0$.

From definition of $\lambda_{0}$, there exist a sequence $\left\{\lambda_{j}\right\}$ such that $\lambda_{j}<\lambda_{0}$ and $\lambda_{j} \rightarrow \lambda_{0}$, and a sequence $\left\{\left(t_{j}, \lambda_{j}\right)\right\}$ of interior minimum points of $w_{\lambda_{j}}$ such that $\left(t_{j}, \theta_{j}\right) \rightarrow\left(t^{*}, \theta^{*}\right)$ with $w_{\lambda_{j}}\left(t_{j}, \theta_{j}\right)<0$. Taking the limit we get $w_{\lambda_{0}}\left(t^{*}, \theta^{*}\right)=0$ and $\nabla w_{\lambda_{0}}\left(t^{*}, \theta^{*}\right)=0$. Therefore $t^{*}=\lambda_{0}$, but this is a contradiction to the Hopf's lemma. This proves the Claim 3.

With these claims on hand, let us prove the Theorem 4.1. By Claim 3, there exist $\lambda_{0}>-\log (3 n)$ and $\theta_{0} \in \mathbb{S}^{n-1}$ such that

$$
w_{\lambda_{0}}\left(-\log \left(\delta \varepsilon^{-1}\right), \theta_{0}\right)=0
$$

Then, by definition of $w_{\lambda_{0}}$ and 4.9 we get

$$
0<c(\delta) \leq v\left(-\log \left(\delta \varepsilon^{-1}\right), \theta_{0}\right)=\left(v_{\lambda_{0}}+2 h_{\lambda_{0}}\right)\left(-\log \left(\delta \varepsilon^{-1}\right), \theta_{0}\right) .
$$

But $v(t, \theta)=O\left(e^{\frac{2-n}{2}(2 \lambda-t)}\right)$ implies that

$$
v\left(-\log \left(\delta \varepsilon^{-1}\right), \theta_{0}\right) \leq c\left(\lambda_{0}, \delta\right) \varepsilon^{\frac{n-2}{2}}
$$

and so

$$
0<c(\delta) \leq c\left(\lambda_{0}, \delta\right) \varepsilon^{\frac{n-2}{2}}+2 h_{\lambda_{0}}\left(-\log \left(\delta \varepsilon^{-1}\right), \theta_{0}\right) .
$$

But by 4.17) we obtain

$$
h_{\lambda_{0}}\left(-\log \left(\delta \varepsilon^{-1}\right), \theta_{0}\right) \leq c \varepsilon^{\frac{1}{2}}
$$

for $n=3$,

$$
h_{\lambda_{0}}\left(-\log \left(\delta \varepsilon^{-1}\right), \theta_{0}\right) \leq c \varepsilon^{1-\gamma}
$$

for $n=4$ and

$$
h_{\lambda_{0}}\left(-\log \left(\delta \varepsilon^{-1}\right), \theta_{0}\right) \leq c \varepsilon^{\frac{1}{2}-\gamma}
$$

for $n=5$, which contradicts 4.25, since we can take the limit $\varepsilon \rightarrow 0$. This completes the proof of the theorem.

We may note that in the proof above we are not using that $-A$ is cooperative. As a consequence of the upper bound we get the following spherical Harnack inequality. 
Corollary 4.2 Suppose $\mathcal{U}$ is a positive smooth solution of (1) in $\Omega=B_{1}^{n}(0) \backslash\{0\}$, $3 \leq n \leq 5$, and assume that the potential A satisfies (H1). Then there exists a constant $c_{1}>0$ such that

$$
\max _{|x|=r} u_{i} \leq c_{1} \min _{|x|=r} u_{i}
$$

for every $0<r<\frac{1}{4}$. Moreover, $\left|\nabla u_{i}\right| \leq c_{1}|x|^{-1} u_{i}$ and $\left|\nabla^{2} u_{i}\right| \leq c_{1}|x|^{-2} u_{i}$.

Proof. Define $u_{i, r}(y)=r^{\frac{n-2}{2}} u_{i}(r y)$, for every $0<r<\frac{1}{4}$ and $|y|<r^{-1}$. Then the upper bound given by theorem (4.1) implies that $u_{i, r}(y) \leq c|y|^{\frac{2-n}{2}}$, for $|y|<\frac{1}{2} r^{-1}$. In particular, if $\frac{1}{2} \leq|y| \leq \frac{3}{2}$, we have that $u_{i, r}(y) \leq 2^{\frac{n-2}{2}} c$.

Moreover

$$
\Delta_{g_{r}} u_{i, r}(y)-r^{2} \sum_{j=1}^{2} A_{i j}(r y) u_{j, r}(y)+\frac{n(n-2)}{4}\left|\mathcal{U}_{r}(y)\right|^{\frac{4}{n-2}} u_{i, r}(y)=0 .
$$

where $\left(g_{r}\right)_{i j}(y)=g_{i j}(r y)$, which implies that

$$
\Delta_{g_{r}} u_{i, r}(y)-r^{2} A_{i i}(r y) u_{i, r}(y)=r^{2} A_{i j}(r y) u_{j, r}(y)-\frac{n(n-2)}{4}\left|\mathcal{U}_{r}(y)\right|^{\frac{4}{n-2}} u_{i, r}(y) .
$$

for $j \neq i$. Using that $-A$ is cooperative, the Harnack inequality for linear elliptic equations and standard elliptic theory imply there exists $c_{1}>0$, not depending on $r$, such that

$$
\max _{|x|=1} u_{i, r} \leq c_{1} \min _{|x|=1} u_{i, r}
$$

and $\left|\nabla u_{i, r}\right|+\left|\nabla^{2} u_{i, r}\right| \leq c_{1} u_{1, r}$ on the sphere of radius 1 . This finishes the proof of the corollary.

\subsection{Pohozaev invariants and removable singularities}

In this section we will define the Pohozaev invariant of a solution and prove a removable singularity theorem for the solutions of the system (1). As a consequence we will derive a fundamental lower bound near the isolated singularity.

Given a positive solution $\mathcal{U}$ to the system (1), we can define $P(r, \mathcal{U})$ similarly to (2.8) by

$$
P(r, \mathcal{U})=\int_{\partial B_{r}}\left(\frac{n-2}{2}\left\langle\mathcal{U}, \frac{\partial \mathcal{U}}{\partial \nu}\right\rangle-\frac{r}{2}|\nabla \mathcal{U}|^{2}+r\left|\frac{\partial \mathcal{U}}{\partial \nu}\right|^{2}+r \frac{(n-2)^{2}}{8}|\mathcal{U}|^{\frac{2 n}{n-2}}\right) d \sigma
$$

The following lemma gives the Pohozaev-type identity we are interested in. 
Lemma 4.3 (Pohozaev Identity) Given $0<s \leq r<1$, it holds

$$
P(r, \mathcal{U})-P(s, \mathcal{U})=-\sum_{i=1}^{2} \int_{B_{r} \backslash B_{s}}\left(x \cdot \nabla u_{i}+\frac{n-2}{2} u_{i}\right)\left(\left(\Delta_{g}-\Delta\right) u_{i}-\sum_{j=1}^{2} A_{i j}(x) u_{j}\right) .
$$

Proof. Adding and subtracting $\Delta u_{i}$ in each system equation, we have

$$
-\Delta u_{i}-\frac{n(n-2)}{4}|\mathcal{U}|^{\frac{4}{n-2}} u_{i}=\left(\Delta_{g}-\Delta\right) u_{i}-\sum_{j=1}^{2} A_{i j}(x) u_{j}
$$

in the punctured ball $\Omega=B_{1}^{n}(0) \backslash\{0\}$. Mutiplying both sides by $x \cdot \nabla u_{i}$ and integrating over $B_{r} \backslash B_{s}$ we have

$$
-\int_{B_{r} \backslash B_{s}} x \cdot \nabla u_{i}\left(\Delta u_{i}+\frac{n(n-2)}{4}|\mathcal{U}|^{\frac{4}{n-2}} u_{i}\right)=\int_{B_{r} \backslash B_{s}} x \cdot \nabla u_{i}\left(\left(\Delta_{g}-\Delta\right)\left(u_{i}\right)-\sum_{j} A_{i j}(x) u_{j}\right) .
$$

First using integration by parts we get

$$
\begin{aligned}
& \int_{B_{r} \backslash B_{s}} x \cdot \nabla u_{i} \Delta u_{i}=\frac{n-2}{2} \int_{B_{r} \backslash B_{s}}\left|\nabla u_{i}\right|^{2}-\frac{r}{2} \int_{\partial B_{r}}\left|\nabla u_{i}\right|^{2}+\frac{s}{2} \int_{\partial B_{s}}\left|\nabla u_{i}\right|^{2} \\
& +r \int_{\partial B_{r}}\left|\frac{\partial u_{i}}{\partial \nu}\right|^{2}-s \int_{\partial B_{s}}\left|\frac{\partial u_{i}}{\partial \nu}\right|^{2} .
\end{aligned}
$$

On the other hand, multiplying each equation for $u_{i}$ and integrating over $B_{r} \backslash B_{s}$ we have

$$
\begin{aligned}
& \int_{B_{r} \backslash B_{s}} u_{i}\left(\left(\Delta_{g}-\Delta\right)\left(u_{i}\right)-\sum_{j=1}^{2} A_{i j}(x) u_{j}\right)=-\int_{B_{r} \backslash B_{s}} u_{i}\left(\Delta u_{i}+\frac{n(n-2)}{4}|\mathcal{U}|^{\frac{4}{n-2}} u_{i}\right)= \\
& \int_{B_{r} \backslash B_{s}}\left|\nabla u_{i}\right|^{2}-\int_{\partial B_{r}} u_{i} \frac{\partial u_{i}}{\partial \nu}+\int_{\partial B_{s}} u_{i} \frac{\partial u_{i}}{\partial \nu}-\frac{n(n-2)}{4} \int_{B_{r} \backslash B_{s}}|\mathcal{U}|^{\frac{4}{n-2}} u_{i}^{2}
\end{aligned}
$$

which implies that

$$
\begin{aligned}
& \int_{B_{r} \backslash B_{s}}\left|\nabla u_{i}\right|^{2}=\int_{\partial B_{r}} u_{i} \frac{\partial u_{i}}{\partial \nu}-\int_{\partial B_{s}} u_{i} \frac{\partial u_{i}}{\partial \nu}+\frac{n(n-2)}{4} \int_{B_{r} \backslash B_{s}}|\mathcal{U}|^{\frac{4}{n-2}} u_{i}^{2} \\
& +\int_{B_{r} \backslash B_{s}} u_{i}\left(\left(\Delta_{g}-\Delta\right)\left(u_{i}\right)-\sum_{j=1}^{2} A_{i j}(x) u_{j}\right)
\end{aligned}
$$

Replacing (4.29) in 4.28), we conclude that

$$
\begin{aligned}
& \int_{B_{r} \backslash B_{s}}\left(x \cdot \nabla u_{i}+\frac{n-2}{2} u_{i}\right)\left(\left(\Delta_{g}-\Delta\right)\left(u_{i}\right)-\sum_{j=1}^{2} A_{i j}(x) u_{j}\right) \\
& =-\int_{\partial B_{r}}\left[\frac{n-2}{2} u_{i} \frac{\partial u_{i}}{\partial \nu}-\frac{r}{2}\left|\nabla u_{i}\right|^{2}+r\left|\frac{\partial u_{i}}{\partial \nu}\right|^{2}\right]+\int_{\partial B_{s}}\left[\frac{n-2}{2} u_{i} \frac{\partial u_{i}}{\partial \nu}-\frac{s}{2}\left|\nabla u_{i}\right|^{2}+s\left|\frac{\partial u_{i}}{\partial \nu}\right|^{2}\right] \\
& -\frac{n(n-2)^{2}}{8} \int_{B_{r} \backslash B_{s}}|\mathcal{U}|^{\frac{2 n}{n-2}}-\frac{n(n-2)}{4} \int_{B_{r} \backslash B_{s}}\left(x \cdot \nabla u_{i}|\mathcal{U}|^{\frac{4}{n-2}} u_{i}\right) .
\end{aligned}
$$


Note also that, using $\frac{n-2}{2 n} \partial_{k}\left(|\mathcal{U}|^{\frac{2 n}{n-2}}\right)=\sum_{i}|\mathcal{U}|^{\frac{4}{n-2}} u_{i} \partial_{k} u_{i}$, we obtain

$$
\begin{aligned}
& -\sum_{i=1}^{2} \int_{B_{r} \backslash B_{s}} x \cdot \nabla u_{i}|\mathcal{U}|^{\frac{4}{n-2}} u_{i}=\frac{n-2}{2} \int_{B_{r} \backslash B_{s}}|\mathcal{U}|^{\frac{2 n}{n-2}}-r \frac{n-2}{2 n} \int_{\partial B_{r}}|\mathcal{U}|^{\frac{2 n}{n-2}} \\
& +s \frac{n-2}{2 n} \int_{\partial B_{s}}|\mathcal{U}|^{\frac{2 n}{n-2}}
\end{aligned}
$$

which finishes the proof.

In the case of the limit system we saw in 2.7 that $P(r, \mathcal{U})$ does not depend on $r$, and therefore is an invariant of the solution $\mathcal{U}$.

In order to define the invariant in a Riemannian setting, we need the upper bounds given by Theorem 4.1. In fact, since $g_{i j}=\delta_{i j}+O\left(|x|^{2}\right)$, we will have

$$
\left|\left(x \cdot \nabla u_{i}+\frac{n-2}{2} u_{i}\right)\left(\left(\Delta-\Delta_{g}\right) u_{i}-\sum_{j=1}^{2} A_{i j}(x) u_{j}\right)\right| \leq c|x|^{2-n},
$$

for each coordinate $i$ and the Pohozaev identity tell us the limit

$$
P(\mathcal{U}):=\lim _{r \rightarrow 0} P(r, \mathcal{U})
$$

exists. The number $P(\mathcal{U})$ is called the Pohozaev invariant of the solution $\mathcal{U}=\left(u_{1}, u_{2}\right)$.

Our main result of this subsection is the following.

Theorem 4.4 Let $\mathcal{U}$ be a positive solution to the system (1) in $B_{1}^{n}(0) \backslash\{0\}, 3 \leq n \leq 5$ and that the potential $A$ satisfies (H1) and (H2). Then $P(\mathcal{U}) \leq 0$. Moreover, $P(\mathcal{U})=0$ if and only if each coordinate $u_{i}$ is smooth on the origin.

The strategy of our proof will be assuming that $P(\mathcal{U}) \geq 0$ and then proving that in this case the origin is a removable singularity, and hence that the invariant is zero. In what follows let us denote by

$$
u(x)=u_{1}(x)+u_{2}(x)
$$

$\bar{u}$ the average of $u$ over $\partial B_{r}$, that is,

$$
\bar{u}(r):=f_{\partial B_{r}} u:=\frac{1}{\operatorname{vol}\left(\partial B_{r}\right)} \int_{\partial B_{r}} u
$$

and define

$$
w(t)=\bar{u}(r) r^{\frac{n-2}{2}}
$$

where $t=-\ln r$.

We have divided the proof into a sequence of lemmas. 
Lemma 4.5 Suppose $\mathcal{U}$ be a positive solution of (1) which satisfies $P(\mathcal{U}) \geq 0$. Then

$$
\liminf _{x \rightarrow 0} u(x)|x|^{\frac{n-2}{2}}=0 .
$$

Proof. If this result is not true, without loss of generality we can suppose that there exist positive constants $c_{1}, c_{2}$ such that

$$
c_{1}|x|^{\frac{2-n}{2}} \leq u_{1}(x) \leq c_{2}|x|^{\frac{2-n}{2}}
$$

where the second inequality above follows from Theorem 4.1. Choose any sequence $r_{k} \rightarrow 0$, and define

$$
u_{i, k}(x)=r_{k}^{\frac{n-2}{2}} u_{i}\left(r_{k} x\right)
$$

Then, using 4.31, we have the following estimate for $u_{1, k}$,

$$
c_{1}|x|^{\frac{2-n}{2}} \leq u_{1, k}(x) \leq c_{2}|x|^{\frac{2-n}{2}} .
$$

Moreover $u_{i, k}$ satisfies

$$
-\Delta_{g_{k}} u_{i, k}+r_{k}^{2} \sum_{j=1}^{2} A_{i j}\left(r_{k} x\right) u_{j, k}=\frac{n(n-2)}{4}\left|\mathcal{U}_{k}\right|^{\frac{4}{n-2}} u_{i, k} \quad \text { in } \quad B_{r_{k}^{-1}}(0) \backslash\{0\},
$$

where $\left(g_{k}\right)_{l m}(x)=g_{l m}\left(r_{k} x\right)$. Elliptic theory then implies that there exists a subsequence, also denoted by $u_{i, k}$, which converges in compact subsets of $\mathbb{R}^{n} \backslash\{0\}$ to a solution $\mathcal{U}_{0}=\left(u_{1,0}, u_{2,0}\right)$ of the limit system

$$
\Delta u_{i, 0}+\frac{n(n-2)}{4}\left|\mathcal{U}_{0}\right|^{\frac{4}{n-2}} u_{i, 0}=0
$$

By 4.32 we get

$$
u_{1,0}(x) \geq c_{1}|x|^{\frac{2-n}{2}},
$$

which implies that $\mathcal{U}_{0}$ is a singular at the origin. However, by Theorem 2.8 we know that $P\left(\mathcal{U}_{0}\right)<0$. This is a contradiction, because

$$
P\left(\mathcal{U}_{0}\right)=P\left(1, \mathcal{U}_{0}\right)=\lim _{k \rightarrow \infty} P\left(1, \mathcal{U}_{k}\right)=\lim _{k \rightarrow \infty} P\left(r_{k}, \mathcal{U}\right)=P(\mathcal{U}) \geq 0
$$

Lemma 4.6 Assume that $\mathcal{U}$ is a positive solution of (1) and that the potential $A$ satisfies (H1) and (H2). Suppose that there exists a sequence $\left(t_{k}\right)_{k \in \mathbb{N}}$ of minimum points for 
$w$ such that $\lim _{k \rightarrow \infty} w\left(t_{k}\right)=0$. Along $|x|=r_{k}$, where $t_{k}=-\ln r_{k}$, there exist positive constants $a_{i}, b_{i}$ such that

$$
\begin{array}{r}
u_{i}(x)=\bar{u}\left(r_{k}\right)\left(c_{i}+o(1)\right) \\
\left|\nabla u_{i}(x)\right|=-\bar{u}^{\prime}\left(r_{k}\right)\left(2 a_{i}+o(1)\right) .
\end{array}
$$

where $c_{i}=a_{i}+b_{i}$. Moreover $\langle a, b\rangle \neq 0$.

Proof. Let $r_{k}=e^{-t_{k}}$ and define $w_{i}=r^{\frac{n-2}{2}} \bar{u}_{i}$ and

$$
v_{i, k}(y)=r_{k}^{\frac{n-2}{2}} u_{i}\left(r_{k} y\right)
$$

Once that $\bar{v}_{i, k}(1)=w_{1}\left(t_{k}\right) \leq w_{1}\left(t_{k}\right)+w_{2}\left(t_{k}\right)=w\left(t_{k}\right) \rightarrow 0$ we get from Harnack inequality that each coordinate $v_{i, k}$ converge uniformly in compact subsets of $\mathbb{R}^{n} \backslash\{0\}$ to 0 . So, if we define

$$
h_{i, k}(y)=v_{k}(p)^{-1} v_{i, k}(y)
$$

where $v_{k}=\sum_{i} v_{i, k}$ and $p=(1,0, \ldots, 0)$, we have that

$$
\begin{aligned}
-\Delta_{g_{k}} h_{i, k}(y) & =-v_{k}(p)^{-1}\left(\Delta_{g_{k}} v_{i, k}\right) \\
& =v_{k}(p)^{-1}\left(\frac{n(n-2)}{4}\left|\mathcal{V}_{k}\right|^{\frac{4}{n-2}} v_{i, k}-r_{k}^{2} \sum_{j=1}^{2} A_{i j}\left(r_{k} y\right) v_{j, k}\right) .
\end{aligned}
$$

This implies

$$
-\Delta_{g_{k}} h_{i, k}+r_{k}^{2} \sum_{j=1}^{2} A_{i j}\left(r_{k} y\right) h_{j, k}=\frac{n(n-2)}{4} v_{k}(p)^{\frac{4}{n-2}}\left|H_{k}\right|^{\frac{4}{n-2}} h_{i, k},
$$

where $\left(g_{i}\right)_{l m}(y)=g_{l m}\left(r_{i} y\right)$ and $H_{k}=\left(h_{1, k}, h_{2, k}\right)$. By elliptic estimates we know that there exists a subsequence $h_{i, k}$ which converge in $C_{l o c}^{2}$ to a nonnegative harmonic function $h_{i}$ in $\mathbb{R}^{n} \backslash\{0\}$. Then

$$
h_{i}(y)=a_{i}|y|^{2-n}+b_{i}
$$

and $a_{1}+a_{2}=b_{1}+b_{2}=\frac{1}{2}$ since $h_{1}(p)+h_{2}(p)=1$ and $\partial_{r}\left(\left(h_{1}+h_{2}\right)(r) r^{\frac{n-2}{2}}\right)=0$ at $r=1$.

Now, multiplying line $i$ of system (1) by $u_{j}$ and integrating over $B_{r_{k}}(0) \backslash B_{\varepsilon r_{k}}(0)$, we obtain that

$$
\int_{B_{r_{k}} \backslash B_{\varepsilon r_{k}}} u_{j} \Delta_{g} u_{i} d v_{g}+\sum_{l=1}^{2} \int_{B_{r_{k}} \backslash B_{\varepsilon r_{k}}} A_{i l} u_{l} d v_{g}=\frac{n(n-2)}{4} \int_{B_{r_{k}} \backslash B_{\varepsilon r_{k}}}|\mathcal{U}|^{\frac{4}{n-2}} u_{i} u_{j} d v_{g} .
$$

Reversing $i$ and $j$ and subtracting one equation from the other, we obtain

$$
\int_{B_{r_{k}} \backslash B_{\varepsilon r_{k}}}\left(u_{j} \Delta_{g} u_{i}-u_{i} \Delta_{g} u_{j}\right) d v_{g}=\sum_{l=1}^{2} \int_{B_{r_{k}} \backslash B_{\varepsilon r_{k}}}\left(u_{i} A_{j l}-u_{j} A_{i l}\right) u_{l} d v_{g},
$$


which, integrating by parts, guarantees that

$$
\int_{\partial B_{r_{k}}}\left(u_{j} \partial_{r} u_{i}-u_{i} \partial_{r} u_{j}\right) d \sigma_{g}=\sum_{l=1}^{2} \int_{B_{r_{k}} \backslash B_{\varepsilon r_{k}}}\left(u_{i} A_{j l}-u_{j} A_{i l}\right) u_{l} d v_{g}+\int_{\partial B_{\varepsilon r_{k}}}\left(u_{j} \partial_{r} u_{i}-u_{i} \partial_{r} u_{j}\right) d \sigma_{g} .
$$

In order to analyse the last integral on the right-hand side, let

$$
\varphi_{i, k}^{\varepsilon}(z)=\left(\varepsilon r_{k}\right)^{\frac{n-2}{2}} u_{i}\left(\varepsilon r_{k} z\right)
$$

Then

$$
\int_{\partial B_{\varepsilon r_{k}}}\left(u_{j} \partial_{r} u_{i}-u_{i} \partial_{r} u_{j}\right) d \sigma_{g}=\int_{\partial B_{1}}\left(\varphi_{j, k}^{\varepsilon} \partial_{r} \varphi_{i, k}^{\varepsilon}-\varphi_{i, k}^{\varepsilon} \partial_{r} \varphi_{i, k}^{\varepsilon}\right) d \sigma_{g} .
$$

On the other hand

$$
-\Delta_{g_{\varepsilon k}} \varphi_{i, k}^{\varepsilon}+\left(\varepsilon r_{k}\right)^{2} \sum_{j=1}^{2} \tilde{A}_{i j}^{\varepsilon} \varphi_{j, k}^{\varepsilon}=c(n)\left|\varphi_{k}^{\varepsilon}\right|^{\frac{4}{n-2}} \varphi_{i, k}^{\varepsilon}
$$

in $B_{\left(\varepsilon r_{k}\right)^{-1}}(0) \backslash\{0\}$ and by theorem 4.1

$$
\left|\varphi_{k}^{\varepsilon}(z)\right| \leq C|z|^{\frac{2-n}{2}} .
$$

Similarly to what we did at the beginning of this proof, after passing to a subsequence, $\varphi_{i, k}^{\varepsilon}$ converges in $C^{2}$ topollogy locally in compact subsets of $\mathbb{R}^{n} \backslash\{0\}$, to a positive solution

$$
\Delta u_{i}+\frac{n(n-2)}{4}|\mathcal{U}|^{\frac{4}{n-2}} u_{i}=0
$$

which using Theorem (2.10) as well Proposition 1.1 in [7] is of the form $u_{0} \Lambda$, where $\Lambda=\left(\Lambda_{1}, \Lambda_{2}\right)$ is a vector in the unit sphere with nonnegative coordinates. Consequently, when $\varepsilon$ goes to zero we have

$$
\lim _{\varepsilon \rightarrow 0} \int_{\partial B_{1}}\left(\varphi_{j, k}^{\varepsilon} \partial_{r} \varphi_{i, k}^{\varepsilon}-\varphi_{i, k}^{\varepsilon} \partial_{r} \varphi_{i, k}^{\varepsilon}\right) d \sigma_{g}=\int_{\partial B_{1}}\left(u_{0} \partial_{r} u_{0} \Lambda_{i} \Lambda_{j}-u_{0} \partial_{r} u_{0} \Lambda_{j} \Lambda_{i}\right) d \sigma_{g}=0 .
$$

Making $\varepsilon \rightarrow 0$ in (4.34, we conclude that

$$
\int_{\partial B_{r_{k}}}\left(u_{j} \partial_{r} u_{i}-u_{i} \partial_{r} u_{j}\right) d \sigma_{g}=\sum_{l=1}^{2} \int_{B_{r_{k}}}\left(u_{i} A_{j l}-u_{j} A_{i l}\right) u_{l} d v_{g}
$$

Using the same notation from the first claim, by the hypothesis under the potential $A$ (H2) and by Theorem 4.1 the equation above implies that

$$
\begin{aligned}
\int_{\partial B_{1}}\left(h_{j, k} \partial_{r} h_{i, k}\right. & \left.-h_{i, k} \partial_{r} h_{j, k}\right) d \sigma_{g} \\
& =r_{k}^{2} \sum_{l=1}^{2} \int_{B_{1}} \frac{\left(u_{i}\left(r_{k} x\right) A_{j l}\left(r_{k} x\right)-u_{j}\left(r_{k} x\right) A_{i l}\left(r_{k} x\right)\right) u_{l}\left(r_{k} x\right)}{u\left(r_{k} p\right)^{2}} d v_{g} \\
& =O\left(r_{k}^{5-n} u\left(r_{k} p\right)^{-2}\right),
\end{aligned}
$$


which converges to zero when dimension is less or equal than five. Finally, we conclude that

$$
\int_{\partial B_{1}}\left(h_{j} \partial_{r} h_{i}-h_{i} \partial_{r} h_{j}\right) d \sigma_{g}=0
$$

and consequently

$$
a_{1} b_{2}=a_{2} b_{1}
$$

which finishes the proof.

Lemma 4.7 Assume that $\mathcal{U}$ is a positive solution of (1) and along $|x|=r_{k}$ the solution satisfies (4.33). Then

$$
P\left(r_{k}, \mathcal{U}\right)=\sigma_{n-1}\left(-4\langle a, b\rangle \frac{\delta^{2}}{2} w^{2}\left(t_{k}\right)+\frac{\delta^{2}}{2}\left(c_{1}^{2}+c_{2}^{2}\right)^{\frac{n}{n-2}}|W|^{\frac{2 n}{n-2}}\left(t_{k}\right)\right)(1+o(1)) .
$$

Proof. Since the solution $\mathcal{U}$ satisfies 4.33 , note that

$$
\left\langle\mathcal{U}, \frac{\partial \mathcal{U}}{\partial \nu}\right\rangle=\sum_{i=1}^{2} \bar{u}\left(r_{k}\right) \bar{u}^{\prime}\left(r_{k}\right)\left(2 c_{i} a_{i}+o(1)\right) .
$$

On the other hand, using that $w_{t}\left(t_{k}\right)=0$ it holds the following equality

$$
\bar{u}^{\prime}\left(r_{k}\right) r_{k}^{\frac{n}{2}}=-\frac{n-2}{2} w\left(t_{k}\right)
$$

Multiplying (4.37) by $\bar{u}\left(r_{k}\right)$, we conclude that

$$
\frac{n-2}{2}\left\langle\mathcal{U}, \frac{\partial \mathcal{U}}{\partial \nu}\right\rangle=-\sum_{i=1}^{2} r_{k}^{1-n} \frac{(n-2)^{2}}{2} w^{2}\left(t_{k}\right)\left(c_{i} a_{i}+o(1)\right) .
$$

Similarly, we also have

$$
\begin{aligned}
-\frac{r}{2}|\nabla \mathcal{U}|^{2}+r\left|\frac{\partial \mathcal{U}}{\partial \nu}\right|^{2} & =r_{k} \sum_{i=1}^{2} \bar{u}^{\prime}\left(r_{k}\right)^{2}\left(2 a_{i}^{2}+o(1)\right) \\
& =r_{k}^{1-n} \sum_{i} \frac{(n-2)^{2}}{2} w^{2}\left(t_{k}\right)\left(a_{i}^{2}+o(1)\right)
\end{aligned}
$$

With these two equalities in hands we get

$$
\begin{array}{r}
P\left(r_{k}, \mathcal{U}\right)=\int_{\partial B_{r_{k}}}\left(\frac{n-2}{2}\left\langle\mathcal{U}, \frac{\partial \mathcal{U}}{\partial \nu}\right\rangle-\frac{r}{2}|\nabla \mathcal{U}|^{2}+r\left|\frac{\partial \mathcal{U}}{\partial \nu}\right|^{2}+r \frac{(n-2)^{2}}{8}|\mathcal{U}|^{\frac{2 n}{n-2}}\right) d \sigma \\
=\sigma_{n-1}\left(-4\langle a, b\rangle \frac{\delta^{2}}{2} w\left(t_{k}\right)^{2}+\frac{\delta^{2}}{2}\left(c_{1}^{2}+c_{2}^{2}\right)^{\frac{n}{n-2}}|W|^{\frac{2 n}{n-2}}\left(t_{k}\right)\right)(1+o(1)),
\end{array}
$$

which finishes the proof. 
Lemma 4.8 Let $\mathcal{U}$ a positive solution of (1) defined in the punctured ball. If

$$
\lim _{|x| \rightarrow 0}|x|^{\frac{n-2}{2}} u(x)=0
$$

then $\mathcal{U}$ extends as a smooth solution to all of $B^{n}$.

Proof. We begin by obtaining upper and lower bounds for the second derivatives of $w$ in terms of $w$. Indeed, observe that the upper bound in Theorem 4.1 implies that $w(t)$ is bounded. Then,

$$
\bar{u}_{r}=f_{\partial B_{r}} u_{r}
$$

and since

$$
w_{t}=-\bar{u}_{r} r^{\frac{n}{2}}-\frac{n-2}{2} w,
$$

we also get that $\left|w_{t}\right|$ is bounded. Derivating again the function $w$ we obtain

$$
w_{t t}=\bar{u}_{r r} r^{\frac{n+2}{2}}+(n-1) \bar{u}_{r} r^{\frac{n}{2}}+\left(\frac{n-2}{2}\right)^{2} w .
$$

Choosing a fixed $s<r$, by the Divergence Theorem, we get

$$
\begin{gathered}
\left(\int_{B_{r} \backslash B_{s}} \Delta u\right)_{r}=\left(\int_{\partial B_{r}} u_{r}\right)_{r}=\left(\int_{\partial B_{1}} u_{r}(r \cdot) r^{n-1}\right)_{r} \\
=\frac{n-1}{r} \int_{\partial B_{1}} u_{r}(r \cdot) r^{n-1}+\int_{\partial B_{1}} u_{r r}(r \cdot) r^{n-1}=\frac{n-1}{r} \int_{\partial B_{r}} u_{r}+r^{n-1} \sigma_{n-1}\left(f_{\partial B_{r}} u_{r}\right)_{r},
\end{gathered}
$$

where $\sigma_{n-1}=\operatorname{vol}\left(\mathbb{S}^{n-1}\right)$. Thus

$$
\begin{aligned}
& \bar{u}_{r r}=\left(f_{\partial B_{r}} u_{r}\right)_{r}=(1-n) r^{-1} f_{\partial B_{r}} u_{r}+\sigma_{n-1}^{-1} r^{1-n}\left(\int_{B_{r} \backslash B_{s}} \Delta u\right)_{r} \\
& =\frac{1-n}{r} \bar{u}_{r}+\sigma_{n-1}^{-1} r^{1-n} \sum_{i=1}^{2}\left(\int_{B_{r} \backslash B_{s}}\left(\left(\Delta-\Delta_{g}\right) u_{i}+\sum_{j=1}^{2} A_{i j} u_{j}-c(n)|\mathcal{U}|^{\frac{4}{n-2}} u_{i}\right)\right)_{r}
\end{aligned}
$$

and so

$$
\bar{u}_{r r}+\frac{n-1}{r} \bar{u}_{r}=f_{\partial B_{r}}\left(\left(\Delta-\Delta_{g}\right) u+\sum_{i, j=1}^{2} A_{i j}(x) u_{j}-c(n)|\mathcal{U}|^{\frac{4}{n-2}} u\right)
$$

where $c(n)=\frac{n(n-2)}{4}$. Replacing in 4.39 we have that

$$
w_{t t}-\left(\frac{n-2}{2}\right)^{2} w=r^{\frac{n+2}{2}} f_{\partial B_{r}}\left(\left(\Delta-\Delta_{g}\right) u+\sum_{i, j=1}^{2} A_{i j}(x) u_{j}-c(n)|\mathcal{U}|^{\frac{4}{n-2}} u\right) \text {. }
$$


Applying the spherical Harnack inequality in Corollary 4.2 for each coordinate function we have that

$$
C^{-1} \bar{u}^{\frac{n+2}{n-2}} \leq c(n) f_{\partial B_{r}}|\mathcal{U}|^{\frac{4}{n-2}} u \leq C \bar{u}^{\frac{n+2}{n-2}}
$$

and

$$
\left|f_{\partial B_{r}}\left(\left(\Delta-\Delta_{g}\right) u+\sum_{i, j=1}^{2} A_{i j}(x) u_{j}\right)\right| \leq c \bar{u} .
$$

With these estimates we obtain the following inequality

$$
-c_{1} w^{\frac{n+2}{n-2}}-c_{3} e^{-2 t} w \leq w_{t t}-\left(\frac{n-2}{2}\right)^{2} w \leq-c_{2} w^{\frac{n+2}{n-2}}+c_{3} e^{-2 t} w .
$$

By hypotesis (4.38) we know that $\lim _{t \rightarrow \infty} w(t)=0$. The strategy is to show that $u \in L_{l o c}^{p}\left(B_{1}^{n}(0)\right)$ for some $p>2 n /(n-2)$ and then by elliptic theory the function $u$ extends smoothly across the origin. Consequently, each coordinate function $u_{i}$ is smooth.

Note that the first inequality in 4.40 implies that there exists $\varepsilon_{0}>0$, such that if $w(t) \leq \varepsilon_{0}$ and $t$ is sufficiently large, then $w_{t t}(t)>0$. Since $\lim _{t \rightarrow \infty} w(t)=0$, there exists $T_{1}$ so that $w(t)<\varepsilon_{0}$ and $w_{t t}>0$ for $t \geq T_{1}$. This implies that $w_{t}<0$ for $t \geq T_{1}$.

By the first inequality in 4.40 , given any positive number $0<\delta<n-2$, there exists $T_{0}$ sufficiently large such that

$$
w_{t t}-\left(\frac{n-2}{2}-\delta\right)^{2} w \geq\left(\delta(n-2)-\delta^{2}-c_{1} w^{\frac{4}{n-2}}-c_{3} e^{-2 t}\right) w \geq 0
$$

for $t \geq T_{0}$, which implies that

$$
\left(w_{t}^{2}-\left(\frac{n-2}{2}-\delta\right)^{2} w^{2}\right)_{t}=2 w_{t}\left(w_{t t}-\left(\frac{n-2}{2}-\delta\right)^{2} w\right) \leq 0
$$

for $t>T_{2}=\max \left\{T_{0}, T_{1}\right\}$, and using that $\lim _{t \rightarrow \infty} w_{t}(t)=0$, we obtain

$$
w_{t}^{2}-\left(\frac{n-2}{2}-\delta\right)^{2} w^{2} \geq 0 .
$$

By integrating we get, for $t \geq T_{2}$, that

$$
w(t) \leq w\left(T_{0}\right) e^{-\left(\frac{n-2}{2}-\delta\right)\left(t-T_{0}\right)} .
$$

Equivalently, there exists $r_{0}(\delta)>0$, so that

$$
u(x) \leq c(\delta)|x|^{-\delta} \text { for all } x \in B_{r_{0}}(0) .
$$


Since $\delta>0$ is arbitrarily small, the estimate above implies that $u \in L_{l o c}^{p}\left(B_{1}(0)\right)$ for arbitrarily large $p$, which finishes our proof.

Now we are ready to prove the main result of this section.

Proof of Theorem 4.4. Following the aforementioned strategy let us suppose that $P(\mathcal{U}) \geq 0$. By Lemma 4.8 the proof is completed by showing that $\lim _{x \rightarrow 0} u(x)|x|^{\frac{n-2}{2}}=0$. Suppose by contradiction that this is false.

Since the Pohozaev invariant is nonnegative, it holds as a consequence of Lemma 4.5 that $\liminf _{x \rightarrow 0} u(x)|x|^{\frac{n-2}{2}}=0$. Then we will assume that $\limsup _{x \rightarrow 0} u(x)|x|^{\frac{n-2}{2}}>0$. Hence we can choose $\varepsilon_{0}>0$ sufficiently small so that we are able to construct sequences $\bar{t}_{k} \leq$ $t_{k} \leq t_{k}^{*}$ satisfying $\lim _{k \rightarrow \infty} \bar{t}_{k}=+\infty, w\left(\bar{t}_{k}\right)=w\left(t_{k}^{*}\right)=\varepsilon_{0}, w_{t}\left(t_{k}\right)=0$ and $\lim _{k \rightarrow \infty} w\left(t_{k}\right)=0$.

Using the results obtained on Lemmas 4.6 and 4.7, we have that

$$
P\left(r_{k}, \mathcal{U}\right)=\sigma_{n-1}\left(-4\langle a, b\rangle \frac{\delta^{2}}{2} w^{2}\left(t_{k}\right)+\frac{\delta^{2}}{2}\left(c_{1}^{2}+c_{2}^{2}\right)^{\frac{n}{n-2}}|W|^{\frac{2 n}{n-2}}\left(t_{k}\right)\right)(1+o(1)),
$$

and consequently

$$
P(\mathcal{U})=\lim _{k \rightarrow \infty} P\left(r_{k}, \mathcal{U}\right)=0 .
$$

Morevover, using that Lemma 4.6 garantees that $\langle a, b\rangle \neq 0$ with the Pohozaev identity, we have the following estimate

$$
w^{2}\left(t_{k}\right) \leq c\left|P\left(r_{k}, \mathcal{U}\right)\right| \leq c\left(I_{1}+I_{2}\right)
$$

where

$$
\begin{gathered}
I_{1}=\int_{B_{r_{k}} \backslash B_{r_{k}^{*}}}|A(\mathcal{U})| d x, \\
I_{2}=\int_{B_{r_{k}^{*}}}|A(\mathcal{U})| d x,
\end{gathered}
$$

and $A(\mathcal{U})=\sum_{i}\left(x \cdot \nabla u_{i}+\frac{n-2}{2} u_{i}\right)\left(\left(\Delta_{g}-\Delta\right)\left(u_{i}\right)-\sum_{j} A_{i j}(x) u_{j}\right)$. We will follow a series of calculations with the goal of obtaining better estimates for the term $I_{1}$. First remember the inequality 4.40 obtained in the proof of Lemma 4.8

$$
-c_{1} w^{\frac{n+2}{n-2}}-c_{3} e^{-2 t} w \leq w_{t t}-\left(\frac{n-2}{2}\right)^{2} w \leq-c_{2} w^{\frac{n+2}{n-2}}+c_{3} e^{-2 t} w
$$

It follows by the first inequality of 4.43 that

$$
w_{t t}-\left(\frac{n-2}{2}\right)^{2} w \geq-c_{1} w^{\frac{n+2}{n-2}}-c_{3} e^{-2 t} w \geq-c_{1} w^{\frac{n+2}{n-2}}-c_{3} e^{-2 t_{k}} w
$$


for $t \geq t_{k}$, which implies

$$
\frac{d}{d t}\left[w_{t}^{2}-\left(\left(\frac{(n-2)}{2}\right)^{2}-c_{3} e^{-2 t_{k}}\right) w^{2}+\frac{n-2}{n} c_{1} w^{\frac{2 n}{n-2}}\right] \geq 0
$$

for $t_{k} \leq t \leq t_{k}^{*}$.

Hence, if $t_{k} \leq t \leq t_{k}^{*}$, then

$$
w_{t}(t)^{2}-g(w(t))+g\left(w\left(t_{k}\right)\right)=\int_{t_{k}}^{t} \frac{d}{d t}\left(w_{t}^{2}-g(w)\right) \geq 0,
$$

which implies that

$$
\frac{d w}{d t} \geq \sqrt{g(w)-g\left(w\left(t_{k}\right)\right)}
$$

and so

$$
t-t_{k}=\int_{w\left(t_{k}\right)}^{w(t)} \frac{d t}{d w} d w \leq \int_{w\left(t_{k}\right)}^{w(t)} \frac{d w}{\sqrt{g(w)-g\left(w\left(t_{k}\right)\right)}}
$$

where

$$
g(w)=\left(\left(\frac{(n-2)}{2}\right)^{2}-c_{3} e^{-2 t_{k}}\right) w^{2}-\frac{n-2}{n} c_{1} w^{\frac{2 n}{n-2}} .
$$

Introducing the variable $\eta=\frac{w(t)}{w\left(t_{k}\right)}$, we get

$$
t-t_{k} \leq \int_{1}^{\frac{w(t)}{w\left(t_{k}\right)}} \frac{d \eta}{\sqrt{\bar{g}(\eta)-\bar{g}(1)}}=\int_{1}^{\frac{w(t)}{w\left(t_{k}\right)}} \sqrt{\frac{\eta^{2}-1}{\bar{g}(\eta)-\bar{g}(1)}} \frac{d \eta}{\sqrt{\eta^{2}-1}}
$$

where

$$
\bar{g}(\eta)=\left(\left(\frac{(n-2)}{2}\right)^{2}-c_{3} e^{-2 t_{k}}\right) \eta^{2}-\frac{n-2}{n} c_{1} w\left(t_{k}\right)^{\frac{4}{n-2}} \eta^{\frac{2 n}{n-2}} .
$$

First, since $1 \leq \eta \leq \frac{w(t)}{w\left(t_{k}\right)} \leq \frac{\varepsilon_{0}}{w\left(t_{k}\right)}$, we have that

$$
\frac{w\left(t_{k}\right)^{\frac{4}{n-2}}\left(\eta^{\frac{2 n}{n-2}}-1\right)}{\eta^{2}-1} \leq c w\left(t_{k}\right)^{\frac{4}{n-2}} \eta^{\frac{4}{n-2}} \leq c \varepsilon_{0}^{\frac{4}{n-2}}
$$

and we observe that

$$
w\left(t_{k}\right)^{\frac{4}{n-2}} \int_{1}^{\frac{w(t)}{w\left(t_{i}\right)}} \frac{\eta^{\frac{4}{n-2}}}{\sqrt{\eta^{2}-1}} d \eta \leq c .
$$

Now

$$
\left(\frac{\eta^{2}-1}{\bar{g}(\eta)-\bar{g}(1)}\right)^{\frac{1}{2}} \leq \frac{2}{n-2}+c e^{-2 t_{k}}+c \frac{w\left(t_{k}\right)^{\frac{4}{n-2}}\left(\eta^{\frac{2 n}{n-2}}-1\right)}{\eta^{2}-1}
$$

Finally, since

$$
\int_{1}^{\frac{w(t)}{w\left(t_{k}\right)}} \frac{d \eta}{\sqrt{\eta^{2}-1}} \leq c+\ln \frac{w(t)}{w\left(t_{k}\right)}
$$


we obtain

$$
\int_{1}^{\frac{w(t)}{w\left(t_{k}\right)}} \frac{d \eta}{\sqrt{\bar{g}(\eta)-\bar{g}(1)}} \leq\left(\frac{2}{n-2}+c e^{-2 t_{k}}\right) \ln \frac{w(t)}{w\left(t_{k}\right)}+c .
$$

From inequality (4.44), we get

$$
t-t_{k} \leq\left(\frac{2}{n-2}+c e^{-2 t_{k}}\right) \ln \frac{w(t)}{w\left(t_{k}\right)}+c
$$

for all $t \in\left(t_{k}, t_{k}^{*}\right)$.

In order to estimate $t-t_{k}$ from below, we first observe that the second inequality in 4.43 implies that

$$
w_{t t}-\left(\left(\frac{n-2}{2}\right)^{2}+c e^{-2 t_{k}}\right) w \leq 0 .
$$

Then the function $w_{t}^{2}-\left(\left(\frac{n-2}{2}\right)^{2}+c e^{-2 t_{k}}\right) w^{2}$ is decreasing in $\left(t_{k}, t_{k}^{*}\right)$, and therefore

$$
w_{t}^{2}-\left(\left(\frac{n-2}{2}\right)^{2}+c e^{-2 t_{k}}\right) w^{2} \leq-\left(\left(\frac{n-2}{2}\right)^{2}+c e^{-2 t_{k}}\right) w^{2}\left(t_{k}\right) .
$$

Hence

$$
w_{t} \leq \sqrt{\left(\left(\frac{n-2}{2}\right)^{2}+c e^{-2 t_{k}}\right)\left(w^{2}-w^{2}\left(t_{k}\right)\right)}
$$

and then

$$
t-t_{k}=\int_{w\left(t_{k}\right)}^{w(t)} \frac{d t}{d w} d w \geq\left(\frac{2}{n-2}-c e^{-2 t_{k}}\right) \int_{w\left(t_{k}\right)}^{w(t)} \frac{d w}{\sqrt{w^{2}-w^{2}\left(t_{k}\right)}} .
$$

Together with inequality (4.45), we get for $t_{k} \leq t \leq t_{k}^{*}$, that

$$
\left(\frac{2}{n-2}-c e^{-2 t_{k}}\right) \ln \frac{w(t)}{w\left(t_{k}\right)} \leq t-t_{k} \leq\left(\frac{2}{n-2}+c e^{-2 t_{k}}\right) \ln \frac{w(t)}{w\left(t_{k}\right)}+c .
$$

Similarly one can prove that, for $\bar{t}_{k} \leq t \leq t_{k}$, it holds

$$
\left(\frac{2}{n-2}-c e^{-2 \bar{t}_{k}}\right) \ln \frac{w(t)}{w\left(t_{k}\right)} \leq t_{k}-t \leq\left(\frac{2}{n-2}+c e^{-2 \bar{t}_{k}}\right) \ln \frac{w(t)}{w\left(t_{k}\right)}+c .
$$

Once we get the above inequalities, lets go back to the estimates of the terms $I_{1}$ and $I_{2}$ in inequality $(4.42)$. Recall that by 4.30$),|A(\mathcal{U})| \leq c|x|^{2-n}$, and therefore

$$
I_{2} \leq c\left(r_{k}^{*}\right)^{2}=c e^{-2 t_{k}^{*}}
$$

From the first inequality in (4.46), we obtain

$$
w(t) \leq w\left(t_{k}\right) \exp \left(\left(\frac{n-2}{2}+c e^{-2 t_{k}}\right)\left(t-t_{k}\right)\right),
$$


which implies

$$
v(x) \leq c w\left(t_{k}\right) \exp \left(-\left(\frac{n-2}{2}+c e^{-2 t_{k}}\right) t_{k}\right) r^{2-n-c e^{-2 t_{k}}}
$$

Recall that, by the spherical Harnack inequality 4.2, for each coordinate function, $u_{i} \leq C r^{\frac{2-n}{2}},\left|\nabla u_{i}\right| \leq C r^{-1} u_{i}$, and $\left|\nabla^{2} u_{i}\right| \leq C r^{-2} u_{i}$, so

$$
|A(\mathcal{U})| \leq C r^{\frac{2-n}{2}} u
$$

Using the estimate 4.48 we obtain

$$
I_{1} \leq c w\left(t_{k}\right) e^{-\frac{n-2}{2} t_{k}-c e^{-2 t_{k} t_{k}}} \int_{B_{r_{k}} \backslash B_{r_{k}^{*}}}|x|^{3-\frac{3 n}{2}-c e^{-2 t_{k}}} d x,
$$

and so

$$
I_{1} \leq c w\left(t_{k}\right) e^{-2 t_{k}}
$$

Therefore, from 4.42 and the estimates for $I_{1}$ and $I_{2}$, we get

$$
w^{2}\left(t_{k}\right) \leq c w\left(t_{k}\right) e^{-2 t_{k}}+c e^{-2 t_{k}^{*}}
$$

Passing to subsequences, if necessary, we can suppose either

$$
w^{2}\left(t_{k}\right) \leq c w\left(t_{k}\right) e^{-2 t_{k}}
$$

or

$$
w^{2}\left(t_{k}\right) \leq c e^{-2 t_{k}^{*}}
$$

Define $L_{k}=-\frac{2}{n-2} \log w\left(t_{k}\right)$ and choose $\delta>0$ small. Then, from the first inequality in 4.47), we get

$$
t_{k}-\bar{t}_{k} \geq(1-\delta) L_{k}-c
$$

and adding to the first inequality in 4.46, we obtain

$$
t_{k}^{*}-\bar{t}_{k} \geq(2-2 \delta) L_{k}-c .
$$

If inequality 4.49 holds, then $w\left(t_{k}\right) \leq c e^{-2 t_{k}}$ and so $L_{k} \geq \frac{4}{n-2} t_{k}-c$. From inequality 4.51, we conclude

$$
t_{k}-t_{k}^{*} \geq(1-\delta) \frac{4}{n-2} t_{k}-c
$$


and consequently

$$
\bar{t}_{k} \leq\left(\frac{n-6}{n-2}+\frac{4 \delta}{n-2}\right) t_{k}+c .
$$

The inequality above gives us a contradiction since $t_{k}^{*} \geq t_{k} \geq \bar{t}_{k} \rightarrow \infty$ as $k \rightarrow \infty$ and on the other hand $\frac{n-6}{n-2}+\frac{4 \delta}{n-2}<0$ by our assumption $3 \leq n \leq 5$.

If inequality 4.50 holds, then $L_{k} \geq \frac{2}{n-2} t_{k}^{*}+c$. From inequality 4.52, we get

$$
\bar{t}_{k} \leq t_{k}^{*}-(2-2 \delta) L_{k}+c
$$

and so

$$
\bar{t}_{k} \leq\left(\frac{n-6}{n-2}+2 \delta\right) t_{k}^{*}+c .
$$

If $3 \leq n \leq 5$, this is again a contradiction by the same reasons as before. Then we conclude that $\lim _{x \rightarrow 0} u(x)|x|^{\frac{n-2}{2}}=0$, and the result follows as consequence of Lemma 4.8 .

As a consequence of the removable singularity theorem, we can now establish a fundamental lower bound.

Corollary 4.9 Assume $3 \leq n \leq 5$ and let $\mathcal{U}$ a positive solution to the system (1) in $B_{1}^{n}(0) \backslash\{0\}$ and that the potential $A$ satisfies (H1) and (H2). If 0 is a nonremovable singularity, then there exists $c>0$ such that

$$
|\mathcal{U}|(x) \geq c d_{g}(x, 0)^{\frac{2-n}{2}}
$$

for $0<d_{g}(x, 0)<\frac{1}{2}$.

Proof. Suppose by contradiction that this is not true. Then $\liminf _{t \rightarrow \infty} w(t)=0$, where $w(t)=r^{\frac{n-2}{2}} \bar{u}(r), u=\sum_{i=1}^{2} u_{i}$ and $t=-\log r$, as in proof of the Theorem 4.4. Since 0 is a nonremovable singularity, we also have $\lim _{\sup _{t \rightarrow \infty}} w(t)>0$, otherwise we contradict Lemma 4.8. Therefore there exists a sequence $t_{k} \rightarrow \infty$ such that $w_{t}\left(t_{k}\right)=0$ and $\lim _{k \rightarrow \infty} w\left(t_{k}\right)=0$. So, if $r_{k}=e^{-t_{k}}$ we can check by Lemmas 4.6 and 4.7

$$
P\left(r_{k}, \mathcal{U}\right)=\sigma_{n-1}\left(-4\langle a, b\rangle \frac{\delta^{2}}{2} w^{2}\left(t_{k}\right)+\frac{\delta^{2}}{2}\left(c_{1}^{2}+c_{2}^{2}\right)^{\frac{n}{n-2}}|W|^{\frac{2 n}{n-2}}\left(t_{k}\right)\right)(1+o(1)) .
$$

where $|W|^{2}=w_{1}^{2}+w_{2}^{2}$. But, in this case

$$
P(\mathcal{U})=\lim _{i \rightarrow \infty} P\left(r_{k}, \mathcal{U}\right)=0
$$

which is a contradiction. This finishes the proof. 


\subsection{Convergence to a Radial Solution}

Our main goal in this section is to prove that a local singular solution to our system is asymptotic to a radial Fowler-type solution, near the nonremovable isolated singularity. The proof will follow exactly the same steps of the Theorem 3.7, however since we are dealing with an arbitrary Riemannian metric we need to take exponential correction terms into account in order to obtain analogous convergence results for the Jacobi fields.

Theorem 4.10 Suppose that $\mathcal{U}$ is a solution of the system (1) in the punctured ball $B_{1}^{n}(0) \backslash\{0\}$ and that the potential $A$ satisfies (H1) and (H2). If there exist positive constants $c_{1}$ and $c_{2}$ such that

$$
c_{1}|x|^{\frac{2-n}{2}} \leq|\mathcal{U}|(x) \leq c_{2}|x|^{\frac{2-n}{2}}
$$

then there exist a Fowler-type solution $\mathcal{U}_{0}=u_{0} \Lambda$ of (6), where $u_{0}$ is a Fowler solution such that

$$
\mathcal{U}(x)=\left(1+O\left(|x|^{\alpha}\right)\right) \mathcal{U}_{0}(x)
$$

as $x \rightarrow 0$, for some $\alpha>0$.

Proof. First we observe that 4.53 implies that the origin is a nonremovable singularity. Thus, by Theorem 4.4 we get that $P(\mathcal{U})<0$. Consider $v_{i}(t, \theta)$ the change of variables from Fowler (1.2) of $u_{i}$.

Let $\left\{\tau_{k}\right\}$ be a sequence of real numbers such that $\tau_{k} \rightarrow \infty$. Consider the translated sequence $v_{i, k}(t, \theta)=v_{i}\left(t+\tau_{k}, \theta\right)$ defined in $\left(-\tau_{k}, \infty\right) \times \mathbb{S}^{n-1}$. By (4.53) we get that

$$
c_{1} \leq\left|\mathcal{V}_{k}(t, \theta)\right| \leq c_{2}
$$

where $\mathcal{V}_{k}=\left(v_{1, k}, v_{2, k}\right)$. Consequently, by standard elliptic estimates, we get the uniform boundedness of any derivative for $t>0$. Since $v_{i, k}$ satisfies,

$$
\mathcal{L}_{g_{k}}\left(v_{i, k}\right)-\sum_{j=1}^{2} B_{i j} v_{j, k}+\frac{n(n-2)}{4}\left|\mathcal{V}_{k}\right|^{\frac{4}{n-2}} v_{i, k}=0
$$

where $\mathcal{L}_{\hat{g_{k}}}$ and $B_{i j}$ are given by 4.5 and $\hat{g}:=d t^{2}+d \theta^{2}+O\left(e^{-2 t}\right) \rightarrow d t^{2}+d \theta^{2}$. Standard elliptic estimates imply that there exists a subsequence, also denoted by $v_{i, k}$, which converges in the $C_{l o c}^{2}$ topology, to a positive solution of

$$
\partial_{t}^{2} v_{i, 0}+\Delta_{\mathbb{S}^{n-1}} v_{i, 0}-\frac{(n-2)^{2}}{4} v_{i, 0}+\frac{n(n-2)}{4}\left|\mathcal{V}_{0}\right|^{\frac{4}{n-2}} v_{i, 0}=0
$$


defined in the whole cylinder. By the characterization result given by Theorem 2.10 , such limit is a Fowler-type solution and we know that there exists a Fowler solution $v_{\varepsilon}$ and a vector in the unit sphere with positive coordinates $\Lambda$ such that $\mathcal{V}_{\varepsilon}(t)=\Lambda v_{\varepsilon}(t)$. Hence $\mathcal{V}_{\varepsilon}$ does not depend on $\theta$, and we necessarily have that any angular derivative $\partial_{\theta} v_{i, k}$ converges uniformly to zero.

Besides, we claim that

$$
\begin{aligned}
v_{i, k}(t, \theta) & =\bar{v}_{i, k}(t)(1+o(1)) \\
\nabla v_{i, k}(t, \theta) & =-\bar{v}_{i, k}^{\prime}(t)(1+o(1)),
\end{aligned}
$$

as $t \rightarrow \infty$. In fact, suppose that the first equality above is false. Then there exist $\bar{\varepsilon}>0$ and sequences $\tau_{k} \rightarrow \infty, \theta_{k} \rightarrow \theta \in \mathbb{S}^{n-1}$ such that

$$
\left|\frac{v_{i, k}\left(\tau_{k}, \theta_{k}\right)}{\bar{v}_{i, k}\left(\tau_{k}\right)}-1\right| \geq \bar{\varepsilon}
$$

for some $i \geq 1$. This is a contradiction because, after passing to a subsequence, $\mathcal{V}_{k}$ converges to a rotationally symmetric Fowler-type solution $\mathcal{V}_{0}$. The second inequality follows from similar arguments.

In the cylindrical setting the Pohozaev integral $P(t, \mathcal{V})=P\left(e^{-t}, \mathcal{U}\right)$ becomes

$$
P(t, \mathcal{V}):=\int_{t \times \mathbb{S}^{n-1}}\left(\frac{1}{2}\left|\partial_{t} \mathcal{V}\right|^{2}-\frac{1}{2}\left|\nabla_{\theta} \mathcal{V}\right|^{2}-\frac{(n-2)^{2}}{8}|\mathcal{V}|^{2}+\frac{(n-2)^{2}}{8}|\mathcal{V}|^{\frac{2 n}{n-2}}\right) d \sigma_{1}
$$

Hence

$$
P\left(\mathcal{V}_{\varepsilon}\right):=P\left(0, \mathcal{V}_{\varepsilon}\right)=\lim _{k \rightarrow \infty} P\left(0, \mathcal{V}_{k}\right)=\lim _{k \rightarrow \infty} P\left(\tau_{k}, \mathcal{V}\right)=P(\mathcal{V})
$$

So we can conclude that the necksize $\varepsilon$ of the limit function is independent of the sequence of numbers $\tau_{k}$. Therefore, for each sequence $\tau_{k} \rightarrow \infty$ the correspondent sequence $\mathcal{V}_{k}$ converges to a function $\mathcal{V}_{\varepsilon, T}(t)=\Lambda v_{\varepsilon}(t+T)$, with $\Lambda \in \mathbb{S}_{+}^{1}$, for some $T \in \mathbb{R}$ which depends on the sequence $\tau_{k}$.

We will show that there exists $T_{0} \in \mathbb{R}$ such that $\mathcal{V}_{k}$ converges to $\mathcal{V}_{\varepsilon, T_{0}}$ for any sequence $\tau_{k} \rightarrow \infty$. The ideia is to use a delicate rescaling argument due originally to Leon Simon. In order to do that we will prove several claims using the Jacobi fields studied in subsection 2.5 as a tool.

Let $T_{\varepsilon}$ be the period of $\mathcal{V}_{\varepsilon}$ and $A_{\tau}=\sup _{t \geq 0}\left|\partial_{\theta} \mathcal{V}_{\tau}\right|$, where $\mathcal{V}_{\tau}(t, \theta)=\mathcal{V}(t+\tau, \theta)$. Note that $A_{\tau}<\infty$, since $\left|\partial_{\theta} \mathcal{V}_{\tau}\right|$ converges uniformly to zero as $t \rightarrow \infty$. 
Claim 1: For every $c>0$, there exists a positive integer $N$ such that, for any $\tau>0$, either

(i) $A_{\tau} \leq c e^{-2 \tau}$ or

(ii) $A_{\tau}$ is attained at some point in $I_{N} \times \mathbb{S}^{n-1}$, where $I_{N}=\left[0, N T_{\varepsilon}\right]$.

Suppose the Claim is not true. Then there exist a constant $c>0$ and sequences $\tau_{k}, s_{k} \rightarrow \infty, \theta_{k} \in \mathbb{S}^{n-1}$ such that $\left|\partial_{\theta} \mathcal{V}_{\tau}\right|\left(s_{k}, \theta_{k}\right)=A_{\tau_{k}}$ and $A_{\tau_{k}}>c e^{-2 \tau_{k}}$ as $k \rightarrow \infty$. Then we can translate back further $s_{k}$ and define $\tilde{v}_{i, k}(t, \theta)=v_{i, k}\left(t+s_{k}, \theta\right)$. Define $\varphi_{i, k}=A_{\tau_{k}}^{-1} \partial_{\theta} \tilde{v}_{i, k}$ and note that $\left|\varphi_{k}\right| \leq 1$, where $\varphi_{\boldsymbol{k}}=\left(\varphi_{1, k}, \varphi_{2, k}\right)$. Now, we have

$$
\mathcal{L}_{\hat{g}_{k}}\left(\tilde{v}_{i, k}\right)-\sum_{j} \tilde{B}_{i j} \tilde{v}_{j, k}+\frac{n(n-2)}{4}\left|\tilde{\mathcal{V}}_{k}\right|^{\frac{4}{n-2}} \tilde{v}_{i, k}=0
$$

where the quantities with tilde are the originals replacing $t$ by $t+\tau_{k}+s_{k}$. This implies that

$$
L_{c y l}\left(\tilde{v}_{i, k}\right)+\frac{n(n-2)}{4}\left|\tilde{\mathcal{V}}_{k}\right|^{\frac{4}{n-2}} \tilde{v}_{i, k}=L_{c y l}\left(\tilde{v}_{i, k}\right)-\mathcal{L}_{\hat{g}_{k}}\left(\tilde{v}_{i, k}\right)+\sum_{j} \tilde{B}_{i j} \tilde{v}_{j, k} .
$$

Taking the derivative with respect to $\theta$ and multipling by $A_{\tau_{k}}^{-1}$, we get

$$
\begin{aligned}
L_{c y l}\left(\varphi_{i, k}\right) & +\frac{n(n-2)}{4}\left|\tilde{\mathcal{V}}_{k}\right|^{\frac{4}{n-2}} \varphi_{i, k}+n\left|\tilde{\mathcal{V}}_{k}\right|^{\frac{4}{n-2}-2} \tilde{v}_{i k}\left\langle\tilde{\mathcal{V}}_{k}, \varphi_{k}\right\rangle \\
& =L_{c y l}\left(\varphi_{i, k}\right)-\mathcal{L}_{\hat{g}_{k}}\left(\varphi_{i, k}\right)+\sum_{j} \tilde{B}_{i j} \varphi_{j, k} .
\end{aligned}
$$

From 4.6 we have

$$
\mathcal{L}_{\hat{g}_{k}}\left(\varphi_{i, k}\right)=\Delta_{\hat{g}_{k}} \varphi_{i, k}-\frac{(n-2)^{2}}{4} \varphi_{i, k}-\frac{n-2}{4} e^{-t} \partial_{r} \log \left|g_{k}\right| \circ \Phi(t, \theta) \varphi_{i, k}
$$

But using the fact $\hat{g}=d t^{2}+d \theta^{2}+O\left(e^{-2 t}\right)$ and the local expression of the laplacian in this metric, we find that

$$
\Delta_{\hat{g}_{k}} \varphi_{i, k}=\partial_{t}^{2} \varphi_{i, k}+\Delta_{\mathbb{S}^{n-1}} \varphi_{i, k}+O\left(e^{-2\left(t+\tau_{k}+s_{k}\right)}\right) .
$$

This implies that

$$
L_{c y l}\left(\varphi_{i, k}\right)+\frac{n(n-2)}{4}\left|\tilde{\mathcal{V}}_{k}\right|^{\frac{4}{n-2}} \varphi_{i, k}+n\left|\tilde{\mathcal{V}}_{k}\right|^{\frac{4}{n-2}-1} \tilde{v}_{i, k}\left\langle\tilde{\mathcal{V}}_{k}, \boldsymbol{\varphi}_{\boldsymbol{k}}\right\rangle=A_{\tau_{k}}^{-1} e^{-2\left(\tau_{k}+s_{k}\right)} O\left(e^{-2 t}\right)
$$

where $\varphi_{\boldsymbol{k}}=\left(\varphi_{1, k}, \varphi_{2, k}\right)$. 
Now we can use elliptic theory to extract a subsequence $\varphi_{i, k}$ which converges in compact subsets to a nontrivial and bounded Jacobi field $\varphi=\left(\varphi_{1}, \varphi_{2}\right)$ which satisfies the following system

$$
L_{c y l}\left(\varphi_{i}\right)+\frac{n(n-2)}{4} v_{\varepsilon}^{\frac{4}{n-2}} \varphi_{i}+n \Lambda_{i}\langle\Lambda, \varphi\rangle v_{\varepsilon}^{\frac{4}{n-2}}=0 .
$$

Since each coordinate function of the limit $\varphi_{i}$ has no zero eingencomponent relative to $\Delta_{\theta}$, we get a contradiction because a Jacobi field with such property is necessarily unbounded. This proves the Claim 1 .

Now suppose we have a sequence $v_{i, k}(t, \theta)$ converging to $\Lambda_{i} v_{\varepsilon}(t+T)$ as $k \rightarrow \infty$. Define

$$
w_{i, k}(t, \theta)=v_{i, k}(t, \theta)-\Lambda_{i} v_{\varepsilon}(t+T) .
$$

Set

$$
\eta_{k}=b \max _{I_{N}}\left|\left(w_{1, k}, w_{2, k}\right)\right|, \quad \bar{\eta}_{k}=\eta_{k}+e^{-(2-\delta) \tau_{k}} \quad \text { and } \quad \varphi_{i, k}=\bar{\eta}_{k}^{-1} w_{i, k},
$$

where $\delta>0$ is a small number and $b>0$ is a fixed number to be chosen later. Note that $\left|\left(\varphi_{1, k}, \varphi_{2, k}\right)\right| \leq b^{-1}$ on $I_{N}$. Then

$$
\mathcal{L}_{\hat{g}_{k}}\left(w_{i, k}\right)+\frac{n(n-2)}{4}\left(\left|\mathcal{V}_{k}\right|^{\frac{4}{n-2}} v_{i, k}-\Lambda_{i} v_{\varepsilon}^{\frac{n+2}{n-2}}\right)=E_{i, k}
$$

where $E_{i, k}=\sum_{j} B_{i j} v_{j, k}+\Lambda_{i}\left(L_{c y l}-\mathcal{L}_{\hat{g}_{k}}\right) v_{\varepsilon}$. First note that by 4.5) we get that $E_{i, k}=O\left(e^{-2\left(\tau_{k}+t\right)}\right)$ when $t \rightarrow \infty$. Second, observe that

$$
\left|\mathcal{V}_{k}\right|^{\frac{4}{n-2}} v_{i, k}-\Lambda_{i} v_{\varepsilon}^{\frac{n+2}{n-2}}=\left|\mathcal{V}_{k}\right|^{\frac{4}{n-2}} w_{i, k}+\Lambda_{i} v_{\varepsilon} \frac{\left|\mathcal{V}_{k}\right|^{\frac{4}{n-2}}-v_{\varepsilon}^{\frac{4}{n-2}}}{\left|\mathcal{V}_{k}\right|^{2}-v_{\varepsilon}^{2}} \sum_{j} w_{j, k}\left(v_{j, k}+\Lambda_{i} v_{\varepsilon}\right)
$$

Multipling (4.56) by $\bar{\eta}_{k}^{-1}$ and taking the limit $k \rightarrow \infty$ we get

$$
L_{c y l}\left(\varphi_{i}\right)+\frac{n(n-2)}{4} v_{0}^{\frac{4}{n-2}} \varphi_{i}+n \Lambda_{i}\langle\Lambda, \varphi\rangle v_{\varepsilon}^{\frac{4}{n-2}}=0,
$$

on the whole cylinder, where $\boldsymbol{\varphi}=\left(\varphi_{1}, \varphi_{2}\right)$ is a Jacobi field.

Claim 2: The Jacobi field $\varphi$ is bounded for $t \geq 0$.

To prove this claim we will use the analysis done in subsection 2.5. By the spectral decomposition for the laplacian in the sphere, we know that it is possible to write the Jacobi field as

$$
\boldsymbol{\varphi}=a_{1} \phi_{\varepsilon, 0}^{1}+a_{2} \phi_{\varepsilon, 0}^{2}+a_{3} \phi_{\varepsilon, 0}^{3}+a_{4} \phi_{\varepsilon, 0}^{4}+\tilde{\boldsymbol{\varphi}}
$$


where $\phi_{\varepsilon, 0}^{i}$ are the linearly independents Jacobi fields corresponding to the eingencomponent independent of $\theta$, and $\tilde{\varphi}$ denotes the projection onto the orthogonal complement. We also know that the functions $\phi_{\varepsilon, 0}^{1}$ and $\phi_{\varepsilon, 0}^{3}$ are bounded and $\phi_{\varepsilon, 0}^{2}$ and $\phi_{\varepsilon, 0}^{4}$ are linearly growing.

Let us show that $\tilde{\boldsymbol{\varphi}}$ is bounded by proving that each $\partial_{\theta} \tilde{\varphi}_{i}=\partial_{\theta} \varphi_{i}$ is bounded for $t \geq 0$. In fact, the function $\partial_{\theta} \varphi_{i}$ is the limit of $\bar{\eta}_{k}^{-1} \partial_{\theta} v_{i, k}$, and we can suppose that $\partial_{\theta} \varphi_{i}$ is nontrivial, otherwise the result is immediate.

If the first item of Claim 1 happens then

$$
\sup _{t \geq 0}\left(\bar{\eta}_{k}^{-1}\left|\partial_{\theta} v_{i, k}\right|\right) \leq \frac{c e^{-2 \tau_{k}}}{\eta_{k}+e^{-(2-\delta) \tau_{k}}} \leq C
$$

While if the second item of Claim 1 is true then

$$
\sup _{t \geq 0}\left(\bar{\eta}_{k}^{-1}\left|\partial_{\theta} v_{i, k}\right|\right) \leq \sup _{t \geq 0}\left(\bar{\eta}_{k}^{-1}\left|\partial_{\theta} \mathcal{V}_{k}\right|\right)=\sup _{t \in I_{N}}\left(\bar{\eta}_{k}^{-1}\left|\partial_{\theta} \mathcal{V}_{k}\right|\right) \leq C
$$

since the sequence $\bar{\eta}_{k}^{-1}\left|\partial_{\theta} \mathcal{V}_{k}\right|$ converges in the $C_{l o c}^{2}$ toplogy. Therefore each $\tilde{\varphi}_{i}$ is bounded for $t \geq 0$, hence exponentially decaying.

To end the proof of the Claim 2 we need to show that $a_{2}=a_{4}=0$. To see this note that the convergence $\varphi_{i, k}=\bar{\eta}_{k}^{-1} w_{i, k} \rightarrow \varphi_{i}$ implies that

$$
\begin{aligned}
\mathcal{V}_{k} & =\Lambda v_{\varepsilon, T}+\bar{\eta}_{k} \boldsymbol{\varphi}+o\left(\bar{\eta}_{k}\right) \\
& =\Lambda v_{\varepsilon, T}+\bar{\eta}_{k}\left(a_{1} \phi_{\varepsilon, 0}^{1}+a_{2} \phi_{\varepsilon, 0}^{2}+a_{3} \phi_{\varepsilon, 0}^{3}+a_{4} \phi_{\varepsilon, 0}^{4}+\tilde{\boldsymbol{\varphi}}\right)+o\left(\bar{\eta}_{k}\right),
\end{aligned}
$$

where $v_{\varepsilon, T}(t)=v_{\varepsilon}(t+T)$. On the other hand by 4.30, 4.55 and the Pohozaev identity, Lemma 4.3 , we have that

$$
P\left(0, \mathcal{V}_{k}\right)=P\left(\tau_{k}, \mathcal{V}\right)=P(\mathcal{V})+O\left(e^{-2 \tau_{k}}\right)=P\left(v_{\varepsilon, T}\right)+O\left(e^{-2 \tau_{k}}\right)
$$

Since $\lim _{k \rightarrow \infty}\left(\bar{\eta}_{k}^{-1} e^{-2 \tau_{k}}\right)=0$, we would have a contradiction in case $a_{2}$ or $a_{4}$ is not zero. Thus each $\varphi$ is bounded for $t \geq 0$.

Now we will show that there exists some $T$ so that the difference between $\mathcal{V}$ and $\mathcal{V}_{\varepsilon, T}=\Lambda v_{\varepsilon, T}$ goes to zero as $t \rightarrow \infty$.

Since we do not know the correct translation parameter, define $\mathcal{V}_{\tau}(t, \theta)=\mathcal{V}(t+$ $\tau, \theta)$ and $\mathcal{W}_{\tau}(t, \theta)=\mathcal{V}_{\tau}(t, \theta)-\Lambda v_{\varepsilon}(t)$. Let $C_{1}>0$ be a fixed constant and consider the interval $I_{N}$ as in the Claim 1. Set also $\eta(\tau)=b \max _{I_{N}}\left|\mathcal{W}_{\tau}\right|$ and $\bar{\eta}(\tau)=\eta(\tau)+e^{-(2-\delta) \tau}$, where $b>0$ is a fixed constant to be chosen later. We observe that $\eta(\tau) \rightarrow 0$ as 
$\tau \rightarrow \infty$. Let us prove the following claim.

Claim 3: If $N, b$ and $\tau$ are sufficiently large and $\bar{\eta}$ is sufficiently small, then there exists $s$ with $|s| \leq C_{1} \bar{\eta}(\tau)$ so that $\bar{\eta}\left(\tau+N T_{\varepsilon}+s\right) \leq \frac{1}{2} \bar{\eta}(\tau)$.

Suppose the claim is not true. Then there exists some sequence $\tau_{k} \rightarrow \infty$ such that $\bar{\eta}\left(\tau_{k}\right) \rightarrow 0$ and for any $s$ satisfying $|s| \leq C_{1} \bar{\eta}\left(\tau_{k}\right)$ we have that $\bar{\eta}\left(\tau_{k}+N T_{\varepsilon}+s\right)>\frac{1}{2} \bar{\eta}\left(\tau_{k}\right)$. Define $\varphi_{i . k}=\bar{\eta}\left(\tau_{k}\right)^{-1} w_{i, \tau_{k}}$, similarly to the previous claim. We can suppose that $\varphi_{i, k}$ converges in $C^{\infty}$ on compact sets to a Jacobi field, which by Claim 2 is bounded for $t \geq 0$. So we can write

$$
\boldsymbol{\varphi}=a_{1} \phi_{\varepsilon, 0}^{1}+a_{3} \phi_{\varepsilon, 0}^{3}+\tilde{\varphi}
$$

where $\tilde{\varphi}$ has exponential decay. Note that $|\varphi| \leq b^{-1}$ on $I_{N}$, which implies that $a_{1}$ and $a_{3}$ are uniformily bounded, independently of the sequence $\tau_{k}$. Moreover, since $\phi_{\varepsilon, 0}^{1}=v_{\varepsilon}^{\prime} \Lambda$ and $\phi_{\varepsilon, 0}^{3}=v_{\varepsilon} \bar{\Lambda}$ we have

$$
\left|a_{3} v_{\varepsilon}\right| \leq|\langle\boldsymbol{\varphi}, \bar{\Lambda}\rangle|+|\langle\tilde{\boldsymbol{\varphi}}, \bar{\Lambda}\rangle| \leq b^{-1}+|\tilde{\boldsymbol{\varphi}}|
$$

on $I_{N}$. We know that $v_{\varepsilon} \geq \varepsilon$ and $\tilde{\varphi}$ decreases exponentially with a fixed rate, and so we can choose $b$ and $N$ sufficiently large such that $\left|a_{3}\right|$ is sufficiently small.

Set $s_{k}=-\bar{\eta}\left(\tau_{k}\right) a_{1}$ whose absolute value is less than $C_{1} \bar{\eta}\left(\tau_{k}\right)$ if we choose $C_{1}$ sufficiently large. Hence for $t \in\left[0,2 N T_{\varepsilon}\right]$ we have

$$
\begin{aligned}
\mathcal{W}_{\tau_{k}+s_{k}}(t, \theta)= & \mathcal{V}\left(t+\tau_{k}-\bar{\eta}\left(\tau_{k}\right) a_{1}, \theta\right)-\Lambda v_{\varepsilon}(t) \\
= & \mathcal{V}_{\tau_{k}}\left(t-\bar{\eta}\left(\tau_{k}\right) a_{1}, \theta\right)-\Lambda v_{\varepsilon}\left(t-\bar{\eta}\left(\tau_{k}\right) a_{1}\right) \\
& -\bar{\eta}\left(\tau_{k}\right) a_{1} \Lambda \frac{v_{\varepsilon}\left(t-\bar{\eta}\left(\tau_{k}\right) a_{1}\right)-v_{\varepsilon}(t)}{-\bar{\eta}\left(\tau_{k}\right) a_{1}} \\
= & \bar{\eta}\left(\tau_{k}\right) \boldsymbol{\varphi}_{k}\left(t-\bar{\eta}\left(\tau_{k}\right) a_{1}, \theta\right)-\bar{\eta}\left(\tau_{k}\right) a_{1} \phi_{\varepsilon, 0}^{1}+o\left(\bar{\eta}\left(\tau_{k}\right)\right) \\
= & \mathcal{W}_{\tau_{k}}(t, \theta)-\bar{\eta}\left(\tau_{k}\right) a_{1} \phi_{\varepsilon, 0}^{1}+o\left(\bar{\eta}\left(\tau_{k}\right)\right),
\end{aligned}
$$

where $\boldsymbol{\varphi}_{k}=\left(\varphi_{1, k}, \varphi_{2, k}\right)$. Here we used the equality $\mathcal{W}_{\tau_{k}}=\bar{\eta}\left(\tau_{k}\right) \boldsymbol{\varphi}_{k}+o\left(\bar{\eta}\left(\tau_{k}\right)\right)$ and the fact that $\boldsymbol{\varphi}_{k}\left(t-\bar{\eta}\left(\tau_{k}\right) a_{1}, \theta\right)-\boldsymbol{\varphi}_{k}(t, \theta)$ goes to zero as $\tau_{k} \rightarrow \infty$.

Consequently, by (4.57), for $t \in\left[0,2 N T_{\varepsilon}\right]$ we get that

$$
\mathcal{W}_{\tau_{k}+s_{k}}=\bar{\eta}\left(\tau_{k}\right) \tilde{\varphi}+\bar{\eta}\left(\tau_{k}\right) a_{3} \phi_{\varepsilon, 0}^{3}+o\left(\bar{\eta}\left(\tau_{k}\right)\right)
$$


which implies

$$
\max _{I_{N}}\left|\mathcal{W}_{\tau_{k}+s_{k}+N T_{\varepsilon}}\right|=\max _{\left[N T_{\varepsilon}, 2 N T_{\varepsilon}\right]}\left|\mathcal{W}_{\tau_{k}+s_{k}}\right| \leq \bar{\eta}\left(\tau_{k}\right) \max _{\left[N T_{\varepsilon}, 2 N T_{\varepsilon}\right]}\left(|\tilde{\boldsymbol{\varphi}}|+\left|a_{3} v_{\varepsilon}\right|\right)+o\left(\bar{\eta}\left(\tau_{k}\right)\right)
$$

Since $\tilde{\boldsymbol{\varphi}}$ decreases exponentially with a fixed rate, by 4.58 we can choose $N$ and $b>0$ suficiently large in a way that the last equality implies that

$$
\max _{I_{N}}\left|\mathcal{W}_{\tau_{k}+s_{k}+N T_{\varepsilon}}\right| \leq \frac{1}{4} \bar{\eta}\left(\tau_{k}\right)
$$

On the other hand, note that

$$
e^{-(2-\delta)\left(\tau_{k}+s_{k}+N T_{\varepsilon}\right)} \leq e^{-(2-\delta) N T_{\varepsilon}} \bar{\eta}\left(\tau_{k}\right) \leq \frac{1}{4} \bar{\eta}\left(\tau_{k}\right)
$$

which implies that $\bar{\eta}\left(\tau+N T_{\varepsilon}+s\right) \leq \frac{1}{2} \bar{\eta}(\tau)$, a contradiction. This ends the proof of the Claim 3.

Once the claim above is proved, using an iterative argument, we are ready to prove that there exists $\sigma$ such that $w_{i, \sigma} \rightarrow 0$ as $t \rightarrow \infty$ for each coordinate. First choose $\tau_{0}$ and $N$ sufficiently large satisfying the Claim 3 and such that $C_{1} \bar{\eta}\left(\tau_{0}\right) \leq \frac{1}{2} N T_{\varepsilon}$. Let $s_{0}=-\bar{\eta}\left(\tau_{0}\right) a_{1}$ be chosen as above. Thus we have $\left|s_{0}\right| \leq C_{1} \bar{\eta}\left(\tau_{0}\right) \leq \frac{1}{2} N T_{\varepsilon}$. Define inductively three sequences by

$$
\begin{aligned}
\sigma_{k} & =\tau_{0}+\sum_{i=0}^{k-1} s_{i} \\
\tau_{k} & =\tau_{k-1}+s_{k-1}+N T_{\varepsilon}=\sigma_{k}+k N T_{\varepsilon} \\
s_{k} & =-\bar{\eta}\left(\tau_{k}\right) a_{1} .
\end{aligned}
$$

By the Claim 3 we get by induction $\bar{\eta}\left(\tau_{k}\right) \leq 2^{-k} \bar{\eta}\left(\tau_{0}\right)$ and $\left|s_{k}\right| \leq 2^{-k-1} N T_{\varepsilon}$. Hence there exists the limit $\sigma=\lim \sigma_{k} \leq \tau_{0}+N T_{\varepsilon}$ and then $\tau_{k} \rightarrow \infty$ as $k \rightarrow \infty$.

We claim $\sigma$ is the correct translation parameter. In fact, choose $k$ such that $t=k N T_{\varepsilon}+[t]$ with $[t] \in I_{N}$, and write

$$
\begin{aligned}
w_{i, \sigma}(t, \theta) & =v_{i}(t+\sigma, \theta)-\Lambda_{i} v_{\varepsilon}(t) \\
& =v_{i}(t+\sigma, \theta)-v_{i}\left(t+\sigma_{k}, \theta\right)+v_{i}\left(t+\sigma_{k}, \theta\right)-\Lambda_{i} v_{\varepsilon}(t) .
\end{aligned}
$$

Since $\partial_{t} v_{i}$ is uniformly bounded, we have

$$
v_{i}(t+\sigma, \theta)-v_{i}\left(t+\sigma_{k}, \theta\right)=\partial_{t} v_{i}\left(t_{0}\right) \sum_{j=k}^{\infty} s_{j}=O\left(2^{-k}\right),
$$


for some $t_{0}$. Besides,

$$
v_{i}\left(t+\sigma_{k}, \theta\right)-\Lambda_{i} v_{\varepsilon}(t)=v_{i}\left(\tau_{k}+[t], \theta\right)-\Lambda_{i} v_{\varepsilon}([t])=w_{i, \tau_{k}}([t], \theta) .
$$

Thus,

$$
\mathcal{W}_{\sigma}(t, \theta)=\mathcal{W}_{\tau_{k}}([t], \theta)+O\left(2^{k}\right)
$$

Since $b \max _{I_{N}}\left|\mathcal{W}_{\tau_{k}}\right|=\eta\left(\tau_{k}\right) \leq \bar{\eta}\left(\tau_{k}\right) \leq 2^{-k} \bar{\eta}\left(\tau_{0}\right)$, it holds $\left|w_{i, \sigma}(t, \theta)\right|=O\left(2^{-k}\right)$ or equivalently, using that $t=k N T_{\varepsilon}+[t]$, we have

$$
\left|w_{i, \sigma}(t, \theta)\right| \leq C_{1} e^{-\frac{\log 2}{N T_{\varepsilon}} t}
$$

which finishes the proof of the theorem.

As a direct consequence of the results proved in this section we have the following corollary.

Corollary 4.11 Suppose that $\mathcal{U}$ is a solution of the system (1) in the punctured ball $B_{1}^{n}(0) \backslash\{0\}$ with the potential $A$ satisfying (H1) and(H2) and $3 \leq n \leq 5$. The there exist a Fowler-type solution $\mathcal{U}_{0}$ from (6) such that

$$
\mathcal{U}(x)=\left(1+O\left(|x|^{\alpha}\right)\right) \mathcal{U}_{0}(x)
$$

as $x \rightarrow 0$, for some $\alpha>0$. 


\section{Bibliography}

[1] Ablowitz, M. J., Prinari, B., And Trubatch, A. D. Discrete and continuous nonlinear Schrödinger systems, vol. 302. Cambridge University Press, 2004.

[2] Akhmediev, N., And Ankiewicz, A. Partially coherent solitons on a finite background. Physical review letters 82, 13 (1999), 2661.

[3] Caffarelli, L. A., Gidas, B., And Spruck, J. Asymptotic symmetry and local behavior of semilinear elliptic equations with critical sobolev growth. Communications on Pure and Applied Mathematics 42, 3 (1989), 271-297.

[4] Chen, C. C., Lin, C.-S., ET AL. Local behavior of singular positive solutions of semilinear elliptic equations with sobolev exponent. Duke Mathematical Journal 78, 2 (1995), 315-334.

[5] Chen, Z., And Lin, C.-S. Removable singularity of positive solutions for a critical elliptic system with isolated singularity. Mathematische Annalen 363, 1-2 (2015), 501-523.

[6] Druet, O., And Hebey, E. Stability for strongly coupled critical elliptic systems in a fully inhomogeneous medium. Analysis $\&$ PDE 2, 3 (2010), 305-359.

[7] Druet, O., Hebey, E., And Vétois, J. Bounded stability for strongly coupled critical elliptic systems below the geometric threshold of the conformal laplacian. Journal of Functional Analysis 258, 3 (2010), 999-1059.

[8] Esry, B., Greene, C. H., Burke Jr, J. P., And Bohn, J. L. Hartree-fock theory for double condensates. Physical Review Letters 78, 19 (1997), 3594. 
[9] Fowler, R. Further studies of emden's and similar differential equations. The Quarterly Journal of Mathematics, 1 (1931), 259-288.

[10] Gidas, B., Ni, W.-M., And NirenberG, L. Symmetry and related properties via the maximum principle. Communications in Mathematical Physics 68, 3 (1979), 209-243.

[11] Korevaar, N., Mazzeo, R., Pacard, F., and Schoen, R. Refined asymptotics for constant scalar curvature metrics with isolated singularities. Inventiones mathematicae 135, 2 (1999), 233-272.

[12] LI, Y. Y. Prescribing scalar curvature on s3, s4 and related problems. Journal of functional analysis 118, 1 (1993), 43-118.

[13] Marques, F. C. Isolated singularities of solutions to the yamabe equation. Calculus of Variations and Partial Differential Equations 32, 3 (2008), 349-371.

[14] Mazzeo, R., And PaCARD, F. Constant scalar curvature metrics with isolated singularities. In Duke Math. J (1999), Citeseer.

[15] Mazzeo, R., Pollack, D., And Uhlenbeck, K. Moduli spaces of singular yamabe metrics. Journal of the American Mathematical Society 9, 2 (1996), 303344.

[16] Pollack, D. Compactness results for complete metrics of constant positive scalar curvature on subdomains of s n. Indiana University Mathematics Journal (1993), $1441-1456$.

[17] Reed, M., And Simon, B. IV: Analysis of Operators, vol. 4. Elsevier, 1978.

[18] Schoen, R. On the number of constant scalar curvature metrics in a conformal class. Differential geometry 52 (1991), 311-320.

[19] Schoen, R. M. The existence of weak solutions with prescribed singular behavior for a conformally invariant scalar equation. Communications on pure and applied mathematics 41, 3 (1988), 317-392. 
[20] Silva Santos, A. A construction of constant scalar curvature manifolds with Delaunay-type ends. Ann. Henri Poincaré 10, 8 (2010), 1487-1535. 